Eur. J. Math. Anal. 2 (2022) 1

doi: $10.28924 /$ ada/ma.2.1

\title{
Convergence and Stability of New Approximation Algorithms for Certain Contractive-Type Mappings
}

\author{
Imo Kalu Agwu*, Donatus Ikechi Igbokwe \\ Department of Mathematics, Micheal Okpara University of Agriculture, Umudike, \\ Umuahia Abia State, Nigeria \\ agwu.imoh@mouau.edu.ng,igbokwedi@yahoo.com \\ *Correspondence: agwu.imoh@mouau.edu.ng
}

\begin{abstract}
AвStRACT. We present new fixed points algorithms called multistep $\mathrm{H}$-iterative scheme and multistep $\mathrm{SH}$-iterative scheme. Under certain contractive-type condition, convergence and stability results were established without any imposition of the 'sum conditions', which to a large extent make some existing iterative schemes so far studied by other authors in this direction practically inefficient. Our results complement and improve some recent results in literature.
\end{abstract}

\section{INTRODUCTION}

There is an intimate connection existing between nonlinear problems and fixed point problems of related contractive-type operators. As a result, researchers have focused more attention on finding approximate fixed points of different contractive-type mappings in recent times; see, for example, [5], [6], [7], [8], [9], [10], [11], [18], [25], [28], etc. and the reference contained in them. Let $X$ be a normed linear space and $\Gamma: X \longrightarrow X$ a given of $X$. We represent the set of fixed points of $\Gamma$ by $F(\Gamma)=\{q \in X: q=\Gamma(q)\}$.

For the past forty years or so, some investigation of fixed points via iterative schemes have been a flourishing area of research for many mathematicians. Mann [21], Ishikawa [22] and Noor [19] iterative schemes, with their modifications, have been studied by different authors and different interesting results were obtained. However, to meet up with the demand of the modern fixed point theory, researchers have continually renewed their efforts toward constructing more efficient iterative schemes. In this direction, following Kirk's introduction of his remarkable iterative scheme in 1971, the results below have found thier place in the current literature.

Let $X$ and $\Gamma$ be as earlier stated.

Received: 1 Nov 2021.

Key words and phrases. strong convergence; multistep $\mathrm{H}$-iterative scheme; multistep sh-iterative scheme; stability; contractive operator; fixed point; normed linear space. 
(a) For arbitrarily $y_{0} \in X$, let the sequence $\left\{y_{n}\right\}_{n=0}^{\infty}$ be defined iteratively as follows:

$$
y_{n+1}=\sum_{j=0}^{\ell} \alpha_{j} \Gamma^{j} y_{n}, \sum_{j=0}^{\ell} \alpha_{j}=1, n \geq 0 .
$$

The iteration method defined by (1.1) is due to Kirk [20].

(b) In [17], Olatinwo presented the algorithms below:

(i) for an arbitrary point $y_{0} \in X$ and for $\alpha_{n, t} \geq 0, \alpha_{n, 0} \neq 0, \alpha_{n, t} \in[0,1]$ and $\ell$ as a fixed integer, define the sequence $\left\{y_{n}\right\}_{n=0}^{\infty}$ by

$$
y_{n+1}=\sum_{t=0}^{\ell} \alpha_{n, t} \Gamma^{t} y_{n}, \sum_{t=0}^{\ell} \alpha_{n, t}=1, n \geq 0
$$

(ii) for an arbitrary point $y_{0} \in X$ and for $\ell \geq m, \alpha_{n, t} \beta_{n, t} \geq 0, \alpha_{n, 0}, \beta_{n, 0} \neq 0, \alpha_{n, t}, \beta_{n, t} \in$ $[0,1]$ and $\ell, m$ as fixed integers, define the sequence $\left\{y_{n}\right\}_{n=0}^{\infty}$ by

$$
\begin{aligned}
y_{n+1} & =\alpha_{n, 0} y_{n}+\sum_{t=0}^{\ell} \alpha_{n, t} \Gamma^{j} z_{n}, \sum_{t=0}^{\ell} \alpha_{n, t}=1 ; \\
z_{n} & =\sum_{t=0}^{m} \beta_{n, t} \Gamma^{t} y_{n}, \sum_{t=0}^{\ell} \beta_{n, t}=1, n \geq 0,
\end{aligned}
$$

and called them Kirk-Mann and Kirk-Ishikawa algorithms, respectively.

(c) Chugh and Kumar [25] presented the following iterative scheme: for an arbitrary point $y_{0} \in X$ and for $\ell \geq m \geq p, \alpha_{n, s}, \gamma_{n, r}, \beta_{n, t} \geq 0, \gamma_{n, 0}, \alpha_{n, 0}, \beta_{n, 0} \neq 0, \alpha_{n, s}, \gamma_{n, r}, \beta_{n, t} \in$ $[0,1]$ and $\ell, m, p$ as fixed integers, define the sequence $\left\{y_{n}\right\}_{n=0}^{\infty}$ by

$$
\begin{aligned}
y_{n+1} & =\gamma_{n, 0} y_{n}+\sum_{r=1}^{\ell} \gamma_{n, r} \Gamma^{r} z_{n}, \sum_{r=0}^{\ell} \gamma_{n, r}=1 ; \\
z_{n} & =\alpha_{n, 0} y_{n}+\sum_{s=1}^{m} \alpha_{n, s} \Gamma^{s} z_{n}, \sum_{s=0}^{m} \alpha_{n, s}=1 ; \\
z_{n} & =\sum_{t=0}^{p} \beta_{n, t} \Gamma^{t} y_{n}, \sum_{t=0}^{p} \beta_{n, t}=1, n \geq 0,
\end{aligned}
$$

(d) Very recently, Akewe, Okeke and Olayiwola [26] presented the following general iterative scheme in the sense of Kirk [20]:

(i) for an arbitrary point $y_{0} \in X$, for $\ell_{1} \geq \ell_{2} \geq \ell_{3} \geq \cdots \geq \ell_{u}$, for each $i, \alpha_{n, s}^{t}, \gamma_{n, t} \geq$ $0, \gamma_{n, 0}, \alpha_{n, 0}, \neq 0$, for each $i, \alpha_{n, s}^{i}, \gamma_{n, t} \in[0,1]$ and $\ell_{1}, \ell_{u}$ as fixed integers for each $u$, 
define the sequence $\left\{y_{n}\right\}_{n=0}^{\infty}$ by

$$
\begin{aligned}
y_{n+1} & =\gamma_{n, 0} y_{n}+\sum_{r=1}^{\ell_{1}} \gamma_{n, r} \Gamma^{r} z_{n}^{1}, \sum_{k=0}^{\ell_{1}} \alpha_{n, r}=1 ; \\
z_{n}^{t} & =\alpha_{n, 0}^{t} y_{n}+\sum_{s=1}^{\ell_{t+1}} \alpha_{n, s}^{t} \Gamma^{j} z_{n}^{t+1}, \sum_{s=0}^{\ell_{t+1}} \alpha_{n, s}^{t}=1, t=1,2, \cdots, u-2 ; \\
z_{n}^{u-1} & =\sum_{s=0}^{\ell_{u}} \alpha_{n, t}^{u-1} \Gamma^{s} y_{n}, \sum_{s=0}^{\ell_{u}} \alpha_{n, t}^{u-1}=1, u \geq 2, n \geq 0,
\end{aligned}
$$

(ii) for an arbitrary point $y_{0} \in X$, retaining the conditions in $(i)$, define the sequence $\left\{y_{n}\right\}_{n=0}^{\infty}$ by

$$
\begin{aligned}
y_{n+1} & =\gamma_{n, 0} z_{n}^{1}+\sum_{r=1}^{\ell_{1}} \gamma_{n, k} \Gamma^{r} z_{n}^{1}, \sum_{r=0}^{\ell_{1}} \alpha_{n, r}=1 \\
z_{n}^{t} & =\alpha_{n, 0}^{t} z_{n}^{t+1}+\sum_{s=1}^{\ell_{t+1}} \alpha_{n, s}^{t} \Gamma^{s} z_{n}^{t+1}, \sum_{s=0}^{\ell_{t+1}} \alpha_{n, s}^{t}=1, t=1,2, \cdots, u-2 \\
z_{n}^{u-1} & =\sum_{s=0}^{\ell_{u}} \alpha_{n, t}^{u-1} \Gamma^{s} y_{n}, \sum_{s=0}^{\ell_{u}} \alpha_{n, t}^{u-1}=1, u \geq 2, n \geq 0
\end{aligned}
$$

It is worthy to mention that in application, the stability of the iterative schemes studied above is quite invaluable. The first researcher to demonstrate this respecting the Banach contraction conditions is Ostrowski [13]. Afterwards, several authors have developed this subject basically because of its indispensable position in the current trend of computer programing. Some recent works in this direction could be seen in [1], [2], [3], [4], [12], [13], [14],

[23], [24], [26] and the references therein.

Remark 1.1. The stability and the convergence results in the papers studied were made possible due to the sum conditions imposed on the control parameters; see, for example, [20], [17], [25], [26], etc and the references therein. But in application, especially for $\mathbb{N}$ large enough, the iterative schemes defined by (1.1), (1.2), (1.3), (1.4) (1.5) and (1.6) become practically inefficient due to the difficulties involved in generating a family of such control parameters, the windy process involved for each sum and the computational cost.

Base on the problems mentioned in Remark 1.1, it becomes necessary to ask the following questions:

Question 1.1. Is it possible to construct an alternative iterative scheme that would address the problems generated by the sum conditions imposed on the control parameters while maintaining, in particular, the results in [26], which in a larger sense contains the results of the other papers studied? 
Following the same argument as in [27] regarding the linear combination of the products of countably finite family of control parameters and the problems mentioned in Remark 1.1, in this paper, we provide an affirmative answer to Question 1.1.

\section{Preliminary}

Throughout the remaining sections, $\phi: \mathbb{R}^{+} \longrightarrow \mathbb{R}^{+}, \mathbb{R}^{+}, \mathbb{N}$ and $H$ will denote monotone increasing subadditive function, the set of positive integers, the set of natural numbers and a real Hilbert space, respectively. Also, the following definition, lemmas and propositions will be needed establish our results.

Definition 2.1. ([13]) Suppose $Y$ is a metric space and let $\Gamma: Y \longrightarrow Y$ be a self-map of $Y$. Let $\left\{x_{n}\right\}_{n=0}^{\infty} \subseteq Y$ be a sequence generated by an iteration scheme

$$
x_{n+1}=g\left(\Gamma, x_{n}\right)
$$

where $x_{0} \in Y$ is the initial approximation and $g$ is some function. Suppeose $\left\{x_{n}\right\}_{n=0}^{\infty}$ converges to a fixed point $q$ of $\Gamma$. Let $\left\{t_{n}\right\}_{n=0}^{\infty} \subseteq Y$ be an arbitrary sequence and set $\epsilon_{n}=d\left(t_{n}, g\left(\Gamma, t_{n}\right)\right), n=$ $1,2, \cdots$ Then, (2.1) is said to be $\Gamma$-stable if and only if $\lim _{n \rightarrow \infty} \epsilon_{n}=0$ implies $\lim _{n \rightarrow \infty} y_{n}=q$.

Note that in practice, the sequence $\left\{t_{n}\right\}_{n=0}^{\infty}$ could be obtained using the following approach: let $x_{0} \in Y$. Set $x_{n+1}=g\left(\Gamma, x_{n}\right)$ and let $t_{0}=x_{0}$. Since, $x_{1}=g\left(\Gamma, x_{0}\right)$ following the rounding in the function $\Gamma$, the value $t_{1}$ (which is estimated to be equal to $x_{1}$ ) could be calculated to give $t_{2}$, an approximate value of $g\left(\Gamma, t_{1}\right)$. The procedure is continued to yield the sequence $\left\{t_{n}\right\}_{n=0}^{\infty}$, which is approximately tha same as the sequence $\left\{x_{n}\right\}_{n=0}^{\infty}$.

Lemma 2.1. (see, e.g., [26]) Let $\left\{\tau_{n}\right\}_{n=0}^{\infty} \in \mathbb{R}^{+}: \tau_{n} \rightarrow 0$ as $n \rightarrow \infty$. For $0 \leq \delta<1$, let $\left\{w_{n}\right\}_{n=0}^{\infty}$ be $a$ sequence of positive numbers satisfying $w_{n+1} \leq \delta w_{n}+\tau_{n}, n=0,1,2, \cdots$ Then, $w_{n} \rightarrow 0$ as $n \rightarrow \infty$.

Lemma 2.2. (see, e.g., [17]) Let (Y,\|.\|) be a normed space, the self-map $\Gamma: Y \longrightarrow Y$ satisfies (1.13) and $\phi: \mathbb{R}^{+} \longrightarrow \mathbb{R}^{+}$(retaining its usual meaning) be such that $\psi(0)=0, \phi(M t)=M \phi(t), M \geq$ $0, t \in \mathbb{R}^{+}$. Then, $\forall i \in \mathbb{N}$ and $\forall s, t \in Y$, we have

$$
\left\|\Gamma^{j} s-\Gamma^{j} t\right\| \leq \rho^{j}\|s-t\|+\sum_{i=0}^{j}\left(\begin{array}{c}
j \\
i
\end{array}\right) \rho^{j-1} \phi(\|s-\Gamma s\|) .
$$

Proposition 2.3. (see,e.g., [27]) Let $\left\{\alpha_{i}\right\}_{i=1}^{\infty} \subseteq \mathbb{N}$, where $k \in\left[0, \mathbb{R}^{+}\right]$is fixed and $N \in \mathbb{N}$ is any integer with $k+1 \leq N$. Then, the following holds:

$$
\alpha_{k}+\sum_{i=k+1}^{N} \alpha_{i} \prod_{j=k}^{i-1}\left(1-\alpha_{j}\right)+\prod_{j=k}^{N}\left(1-\alpha_{j}\right)=1
$$


Proposition 2.4. (see,e.g., [27]) Let $t, u, v \in H$. Let $k \in\left[0, \mathbb{R}^{+}\right]$be fixed and $N \in \mathbb{N}$ be such that $k+1 \leq N$. Let $\left\{v_{i}\right\}_{i=1}^{N-1} \subseteq H$ and $\left\{\alpha_{i}\right\}_{i=1}^{N} \subseteq[0,1]$. Define

$$
y=\alpha_{k} t+\sum_{i=k+1}^{N} \alpha_{i} \prod_{j=k}^{i-1}\left(1-\alpha_{j}\right) v_{i-1}+\prod_{j=k}^{N}\left(1-\alpha_{j}\right) v .
$$

Then,

$$
\begin{aligned}
\|y-u\|^{2}= & \alpha_{k}\|t-u\|^{2}+\sum_{i=k+1}^{N} \alpha_{i} \prod_{j=k}^{i-1}\left(1-\alpha_{j}\right)\left\|v_{i-1}-u\right\|^{2}+\prod_{j=k}^{N}\left(1-\alpha_{j}\right)\|v-u\|^{2} \\
& -\alpha_{k}\left[\sum_{i=k+1}^{N} \alpha_{i} \prod_{j=k}^{i-1}\left(1-\alpha_{j}\right)\left\|t-v_{i-1}\right\|^{2}+\prod_{j=k}^{i-1}\left(1-\alpha_{j}\right)\|t-v\|^{2}\right] \\
& -\left(1-\alpha_{k}\right)\left[\sum_{i=k+1}^{N} \alpha_{i} \prod_{j=k}^{i-1}\left(1-\alpha_{j}\right)\left\|v_{i-1}-\left(\alpha_{i+1}+w_{i+1}\right)\right\|^{2}\right. \\
& \left.+\alpha_{N} \prod_{j=k}^{i-1}\left(1-\alpha_{j}\right)\left\|v-v_{N-1}\right\|^{2}\right]
\end{aligned}
$$

where $w_{k}=\sum_{i=k+1}^{N} \alpha_{i} \prod_{j=k}^{i-1}\left(1-\alpha_{j}\right) v_{i-1}+\prod_{j=k}^{i-1}\left(1-\alpha_{j}\right) v, k=1,2, \cdots, N$ and $w_{n}=\left(1-c_{n}\right) v$.

\section{Main Results I}

Let $H$ be a Hilbert space and let $\Gamma: H \longrightarrow H$ be a self-map of $X$. For arbitrary $x_{0} \in H$ define the sequence $\left\{x_{n+1}\right\}_{n=0}^{\infty}$ iteratively, for $s=1,2, \cdots, k-2$, as follows:

$$
\left\{\begin{array}{l}
x_{n+1}=\delta_{n, 1} x_{n}+\sum_{j=2}^{\ell_{1}} \delta_{n, j} \prod_{i=1}^{j-1}\left(1-\delta_{n, i}\right) \Gamma^{j-1} y_{n}^{1}+\prod_{i=1}^{\ell_{1}}\left(1-\delta_{n, i}\right) \Gamma^{\ell_{1}} y_{n}^{1} \\
y_{n}^{s}=\alpha_{n, 1}^{s} x_{n}+\sum_{j=2}^{\ell_{s+1}} \alpha_{n, j}^{s} \prod_{i=1}^{j-1}\left(1-\alpha_{n, i}^{s}\right) \Gamma^{j-1} y_{n}^{s+1}+\prod_{i=1}^{\ell_{s+1}}\left(1-\alpha_{n, i}^{s}\right) \Gamma^{\ell_{1}} y_{n}^{s+1} \\
y_{n}^{k-1}=\sum_{j=1}^{\ell_{k}} \alpha_{n, j}^{k-1} \prod_{i=1}^{j-1}\left(1-\alpha_{n, i}^{k-1}\right) \Gamma^{j-1} x_{n}+\prod_{i=1}^{\ell_{k}}\left(1-\alpha_{n, i}^{k-1}\right) \Gamma^{\ell_{k}} x_{n}, k \geq 2, n \geq 1,
\end{array}\right.
$$

where $\ell_{1} \geq \ell_{2} \geq \ell_{3} \geq \cdots \geq \ell_{k}$, for each $s,\left\{\left\{\delta_{n, i}\right\}_{n=0}^{\infty}\right\}_{j=1}^{\ell_{k}},\left\{\left\{\alpha_{n, i}\right\}_{n=0}^{\infty}\right\}_{j=1}^{\ell_{k}} \in[0,1]$ for each $k$ and $\ell_{1}, \ell_{2}, \cdots, \ell_{k}$ are fixed integers (for each $k$ ). We shall call the iteration scheme defined by (3.1) the multistep $\mathrm{IH}$-iteration scheme.

Again, for any $x_{0} \in X$, we shall call the sequence $\left\{x_{n}\right\}_{n=0}^{\infty}$ defined recursively, for $s=$ $1,2, \cdots, k-2$, by

$$
\left\{\begin{array}{l}
x_{n+1}=\delta_{n, 1} y_{n}^{1}+\sum_{j=2}^{\ell_{1}} \delta_{n, j} \prod_{i=1}^{j-1}\left(1-\delta_{n, i}\right) \Gamma^{j-1} y_{n}^{1}+\prod_{i=1}^{\ell_{1}}\left(1-\delta_{n, i}\right) \Gamma^{\ell_{1}} y_{n}^{1} \\
y_{n}^{s}=\alpha_{n, 1}^{s} y_{n}^{s+1}+\sum_{j=2}^{\ell_{s}+1} \alpha_{n, j}^{s} \prod_{i=1}^{j-1}\left(1-\alpha_{n, i}^{s}\right) \Gamma^{j-1} y_{n}^{s+1}+\prod_{i=1}^{\ell_{s+1}}\left(1-\alpha_{n, i}^{s}\right) \Gamma^{\ell_{1}} y_{n}^{s+1} \\
y_{n}^{k-1}=\sum_{j=1}^{\ell_{k}} \alpha_{n, j}^{k-1} \prod_{i=1}^{j-1}\left(1-\alpha_{n, i}^{k-1}\right) \Gamma^{j-1} x_{n}+\prod_{i=1}^{\ell_{k}}\left(1-\alpha_{n, i}^{k-1}\right) \Gamma^{\ell_{k}} x_{n}, k \geq 2, n \geq 1
\end{array}\right.
$$

where $\ell_{1} \geq \ell_{2} \geq \ell_{3} \geq \cdots \geq \ell_{k}$, for each $s,\left\{\left\{\delta_{n, i}\right\}_{n=0}^{\infty}\right\}_{j=1}^{\ell_{k}},\left\{\left\{\alpha_{n, i}\right\}_{n=0}^{\infty}\right\}_{j=1}^{\ell_{k}} \in[0,1]$ for each $k$ and $\ell_{1}, \ell_{2}, \cdots, \ell_{k}$ are fixed integers (for each $k$ ), the multistep Dl-iteration scheme. 
Theorem 3.1. Let $H$ be a Hilbert space, $\Gamma: H \longrightarrow H$ be a self-map of $H$ satisfying the contractive condition

$$
\left\|\Gamma^{j} x-\Gamma^{j} y\right\| \leq \rho^{j}\|x-y\|+\sum_{i=0}^{j}\left(\begin{array}{c}
j \\
i
\end{array}\right) \rho^{j-i} \phi(\|x-\Gamma x\|),
$$

where $x, y \in H, 0 \leq \rho^{j}<1$, and let $\phi$ retain its usual meaning with $\phi(0)=0$ and $\phi(M t)=$ $M \phi(t), M \geq 0, t \in \mathbb{R}^{+}$. For arbitrary $x_{0} \in H$, let $\left\{\omega_{n}\right\}_{n=0}^{\infty}$ be the multistep H-iteration scheme defined by (3.1). Then,

(i) $\Gamma$ defined by (3.3) has a fixed point q;

(ii) the multistep $/ \mathrm{H}$-iteration scheme converges strongly to $q \in \Gamma$.

Proof. Firstly, we show that $\Gamma$ satisfying condition of (3.3) has a fixed point. Assume there exists two points $q_{1}, q_{2} \in F(\Gamma)$ with $0<\left\|q_{1}-q_{2}\right\|$. Then, we have

$$
\begin{aligned}
0<\left\|q_{1}-q_{2}\right\|=\left\|\Gamma^{j} q_{1}-\Gamma^{j} q_{2}\right\| & \leq \rho^{j}\left\|q_{1}-q_{2}\right\|+\sum_{i=0}^{j}\left(\begin{array}{c}
j \\
i
\end{array}\right) \rho^{j-i} \phi\left(\left\|q \sqrt{1}-\Gamma q_{1}\right\|\right) \\
& =\rho^{j}\left\|q_{1}-q_{2}\right\|+\sum_{i=0}^{j}\left(\begin{array}{c}
j \\
i
\end{array}\right) \rho^{j-i} \phi(0)
\end{aligned}
$$

$\Rightarrow\left(1-\rho^{j}\right) \rho^{j}\left\|q_{1}-q_{2}\right\| \leq 0$. Using the fact that $\rho^{j} \in\left[[0,1)\right.$, we get $0<1-\rho^{j}$ and $\left\|q_{1}-q_{2}\right\| \leq 0$. Since the norm is a nonnegative function, we get $\left\|q_{1}-q_{2}\right\|=0 ; q_{1}=q_{2}=q($ say). Therefore, $\Gamma$ converges uniquely to a point of $F(\Gamma)$.

Now, we show that the sequence defined by (3.1) converges strongly to $q \in F(\Gamma)$. Using (3.3) and Proposition 2.4 with $x_{n+1}=y, u=q, x_{n}=t, j=i, k=1, \Gamma^{j-1} y_{n}^{1}=v_{j-1}$ and $\Gamma^{\ell_{1}} y_{n}^{1}=v$, we have

$$
\begin{aligned}
\left\|x_{n+1}-q\right\|^{2} \leq & \delta_{n, 1}\left\|x_{n}-q\right\|^{2}+\sum_{j=2}^{\ell_{1}} \delta_{n, j} \prod_{i=1}^{j-1}\left(1-\delta_{n, i}\right)\left\|\Gamma^{j-1} y_{n}^{1}-\Gamma^{j-1} q\right\|^{2} \\
& +\prod_{i=1}^{\ell_{1}}\left(1-\delta_{n, i}\right)\left\|\Gamma^{\ell_{1}} y_{n}^{1}-\Gamma^{\ell_{1}} q\right\|^{2}
\end{aligned}
$$

But from (3.3), with $y=y_{n}^{1}$, we have

$$
\begin{aligned}
\left\|\Gamma^{j-1} y_{n}^{1}-\Gamma^{j-1} q\right\| & \leq \rho^{j}\left\|y_{n}^{1}-q\right\|+\sum_{i=0}^{j}\left(\begin{array}{c}
j \\
i
\end{array}\right) \rho^{j-1} \phi(\|q-\Gamma q\|) \\
& =\rho^{j}\left\|y_{n}^{1}-q\right\|
\end{aligned}
$$


Proposition 2.3, (3.4) and (3.5) imply

$$
\begin{aligned}
\left\|x_{n+1}-q\right\|^{2} \leq & \delta_{n, 1}\left\|x_{n}-q\right\|^{2}+\sum_{j=2}^{\ell_{1}} \delta_{n, j}\left(\rho^{j}\right)^{2} \prod_{i=1}^{j-1}\left(1-\delta_{n, i}\right)\left\|y_{n}^{1}-q\right\|^{2} \\
& +\prod_{i=1}^{\ell_{1}}\left(1-\delta_{n, i}\right)\left(\rho^{j}\right)^{2}\left\|y_{n}^{1}-q\right\|^{2} \\
= & \delta_{n, 1}\left\|x_{n}-q\right\|^{2}+\left(1-\delta_{n, 1}^{1}-\prod_{i=1}^{\ell_{1}}\left(1-\delta_{n, i}\right)\left(\rho^{j}\right)^{2}\right)\left\|y_{n}^{1}-q\right\|^{2} \\
& +\prod_{i=1}^{\ell_{1}}\left(1-\delta_{n, i}\right)\left(\rho^{j}\right)^{2}\left\|y_{n}^{1}-q\right\|^{2} \\
= & \delta_{n, 1}\left\|x_{n}-q\right\|^{2}+\left(1-\delta_{n, 1}^{1}\right)\left\|y_{n}^{1}-q\right\|^{2}
\end{aligned}
$$

Since $\ell_{1}, \ell_{k}$ are fixed integers and $\alpha_{n, i}^{s} \in[0,1]$ for each $s$, we have, using Proposition 2.3, the following estimates for $n=1,2, \cdots$ and $1 \leq s \leq k-1$ :

$$
\begin{aligned}
\left\|y_{n}^{1}-q\right\|^{2} \leq & \alpha_{n, 1}\left\|x_{n}-q\right\|^{2}+\sum_{j=2}^{\ell_{2}} \alpha_{n, j} \prod_{i=1}^{j-1}\left(1-\alpha_{n, i}\right)\left\|\Gamma^{j-1} y_{n}^{2}-\Gamma^{j-1} q\right\|^{2} \\
& +\prod_{i=1}^{\ell_{2}}\left(1-\alpha_{n, i}\right)\left\|\Gamma^{\ell_{2}} y_{n}^{2}-\Gamma^{\ell_{2}} q\right\|^{2} \\
\leq & \alpha_{n, 1}^{1}\left\|x_{n}-q\right\|^{2}+\sum_{j=2}^{\ell_{2}} \alpha_{n, j}\left(\rho^{j}\right)^{2} \prod_{i=1}^{j-1}\left(1-\alpha_{n, i}\right)\left\|y_{n}^{2}-q\right\|^{2} \\
& +\prod_{i=1}^{\ell_{2}}\left(1-\alpha_{n, i}\right)\left(\rho^{j}\right)^{2}\left\|y_{n}^{2}-q\right\|^{2} \\
\leq & \alpha_{n, 1}^{1}\left\|x_{n}-q\right\|^{2}+\sum_{j=2}^{\ell_{2}} \alpha_{n, j}^{1}\left(\rho^{j}\right)^{2} \prod_{i=1}^{j-1}\left(1-\alpha_{n, i}^{1}\right)\left[\alpha_{n, 1}^{2}\left\|x_{n}-q\right\|^{2}\right. \\
& \left.+\sum_{j=2}^{\ell_{3}} \alpha_{n, j}^{2}\left(\rho^{j}\right)^{2} \prod_{i=1}^{j-1}\left(1-\alpha_{n, i}^{2}\right)\left\|y_{n}^{3}-q\right\|^{2}+\prod_{i=1}^{\ell_{3}}\left(1-\alpha_{n, i}^{2}\right)\left(\rho^{j}\right)^{2}\left\|y_{n}^{3}-q\right\|^{2}\right] \\
& +\prod_{i=1}^{\ell_{2}}\left(1-\alpha_{n, i}^{1}\right)\left(\rho^{j}\right)^{2}\left[\alpha_{n, 1}^{2}\left\|x_{n}-q\right\|^{2}+\sum_{j=2}^{\ell_{3}} \alpha_{n, j}^{2}\left(\rho^{j}\right)^{2} \prod_{i=1}^{j-1}\left(1-\alpha_{n, i}^{2}\right)\left\|y_{n}^{3}-q\right\|^{2}\right. \\
& \left.+\prod_{i=1}^{\ell_{3}}\left(1-\alpha_{n, i}^{2}\right)\left(\rho^{j}\right)^{2}\left\|y_{n}^{3}-q\right\|^{2}\right]
\end{aligned}
$$




$$
\begin{aligned}
& =\alpha_{n, 1}^{1}\left\|x_{n}-q\right\|^{2}+\sum_{j=2}^{\ell_{2}} \alpha_{n, j}^{1}\left(\rho^{j}\right)^{2} \prod_{i=1}^{j-1}\left(1-\alpha_{n, i}^{1}\right) \alpha_{n, 1}^{2}\left\|x_{n}-q\right\|^{2} \\
& +\left(\sum_{j=2}^{\ell_{2}} \alpha_{n, j}^{1}\left(\rho^{j}\right)^{2} \prod_{i=1}^{j-1}\left(1-\alpha_{n, i}^{1}\right)\right)\left(\sum_{j=2}^{\ell_{3}} \alpha_{n, j}^{2}\left(\rho^{j}\right)^{2} \prod_{i=1}^{j-1}\left(1-\alpha_{n, i}^{2}\right)\right)\left\|y_{n}^{3}-q\right\|^{2} \\
& +\left(\sum_{j=2}^{\ell_{2}} \alpha_{n, j}^{1}\left(\rho^{j}\right)^{2} \prod_{i=1}^{j-1}\left(1-\alpha_{n, i}^{1}\right)\right)\left(\prod_{i=1}^{\ell_{3}}\left(1-\alpha_{n, i}^{2}\right)\left(\rho^{j}\right)^{2}\right)\left\|y_{n}^{3}-q\right\|^{2} \\
& +\prod_{i=1}^{\ell_{2}}\left(1-\alpha_{n, i}^{1}\right)\left(\rho^{j}\right)^{2} \alpha_{n, 1}^{2}\left\|x_{n}-q\right\|^{2} \\
& +\left(\sum_{j=2}^{\ell_{3}} \alpha_{n, j}^{2}\left(\rho^{j}\right)^{2} \prod_{i=1}^{j-1}\left(1-\alpha_{n, i}^{2}\right)\right)\left(\prod_{i=1}^{\ell_{2}}\left(1-\alpha_{n, i}^{1}\right)\left(\rho^{j}\right)^{2}\right)\left\|y_{n}^{3}-q\right\|^{2} \\
& +\left(\prod_{i=1}^{\ell_{3}}\left(1-\alpha_{n, i}^{2}\right)\left(\rho^{j}\right)^{2}\right)\left(\prod_{i=1}^{\ell_{2}}\left(1-\alpha_{n, i}^{1}\right)\left(\rho^{j}\right)^{2}\right)\left\|y_{n}^{3}-q\right\|^{2} \\
& \leq \alpha_{n, 1}^{1}\left\|x_{n}-q\right\|^{2}+\sum_{j=2}^{\ell_{2}} \alpha_{n, j}^{1}\left(\rho^{j}\right)^{2} \prod_{i=1}^{j-1}\left(1-\alpha_{n, i}^{1}\right) \alpha_{n, 1}^{2}\left\|x_{n}-q\right\|^{2} \\
& +\prod_{i=1}^{\ell_{2}}\left(1-\alpha_{n, i}^{1}\right)\left(\rho^{j}\right)^{2} \alpha_{n, 1}^{2}\left\|x_{n}-q\right\|^{2} \\
& +\left(\sum_{j=2}^{\ell_{2}} \alpha_{n, j}^{1}\left(\rho^{j}\right)^{2} \prod_{i=1}^{j-1}\left(1-\alpha_{n, i}^{1}\right)\right)\left(\sum_{j=2}^{\ell_{3}} \alpha_{n, j}^{2}\left(\rho^{j}\right)^{2} \prod_{i=1}^{j-1}\left(1-\alpha_{n, i}^{2}\right)\right)\left[\alpha_{n, 1}^{3}\left\|x_{n}-q\right\|^{2}\right. \\
& \left.+\sum_{j=2}^{\ell_{4}} \alpha_{n, j}^{3}\left(\rho^{j}\right)^{2} \prod_{i=1}^{j-1}\left(1-\alpha_{n, i}^{3}\right)\left\|y_{n}^{4}-q\right\|^{2}+\prod_{i=1}^{\ell_{4}}\left(1-\alpha_{n, i}^{4}\right)\left(\rho^{j}\right)^{2}\left\|y_{n}^{4}-q\right\|^{2}\right] \\
& +\left(\sum_{j=2}^{\ell_{2}} \alpha_{n, j}^{1}\left(\rho^{j}\right)^{2} \prod_{i=1}^{j-1}\left(1-\alpha_{n, i}^{1}\right)\right)\left(\prod_{i=1}^{\ell_{3}}\left(1-\alpha_{n, i}^{2}\right)\left(\rho^{j}\right)^{2}\right)\left[\alpha_{n, 1}^{3}\left\|x_{n}-q\right\|^{2}\right. \\
& \left.+\sum_{j=2}^{\ell_{4}} \alpha_{n, j}^{3}\left(\rho^{j}\right)^{2} \prod_{i=1}^{j-1}\left(1-\alpha_{n, i}^{3}\right)\left\|y_{n}^{4}-q\right\|^{2}+\prod_{i=1}^{\ell_{4}}\left(1-\alpha_{n, i}^{4}\right)\left(\rho^{j}\right)^{2}\left\|y_{n}^{4}-q\right\|^{2}\right] \\
& +\left(\sum_{j=2}^{\ell_{3}} \alpha_{n, j}^{2}\left(\rho^{j}\right)^{2} \prod_{i=1}^{j-1}\left(1-\alpha_{n, i}^{2}\right)\right)\left(\prod_{i=1}^{\ell_{2}}\left(1-\alpha_{n, i}^{1}\right)\left(\rho^{j}\right)^{2}\right)\left[\alpha_{n, 1}^{3}\left\|x_{n}-q\right\|^{2}\right. \\
& \left.+\sum_{j=2}^{\ell_{4}} \alpha_{n, j}^{3}\left(\rho^{j}\right)^{2} \prod_{i=1}^{j-1}\left(1-\alpha_{n, i}^{3}\right)\left\|y_{n}^{4}-q\right\|^{2}+\prod_{i=1}^{\ell_{4}}\left(1-\alpha_{n, i}^{4}\right)\left(\rho^{j}\right)^{2}\left\|y_{n}^{4}-q\right\|^{2}\right] \\
& +\left(\prod_{i=1}^{\ell_{3}}\left(1-\alpha_{n, i}^{2}\right)\left(\rho^{j}\right)^{2}\right)\left(\prod_{i=1}^{\ell_{2}}\left(1-\alpha_{n, i}^{1}\right)\left(\rho^{j}\right)^{2}\right)\left[\alpha_{n, 1}^{3}\left\|x_{n}-q\right\|^{2}\right. \\
& \left.+\sum_{j=2}^{\ell_{4}} \alpha_{n, j}^{3}\left(\rho^{j}\right)^{2} \prod_{i=1}^{j-1}\left(1-\alpha_{n, i}^{3}\right)\left\|y_{n}^{4}-q\right\|^{2}+\prod_{i=1}^{\ell_{4}}\left(1-\alpha_{n, i}^{4}\right)\left(\rho^{j}\right)^{2}\left\|y_{n}^{4}-q\right\|^{2}\right]
\end{aligned}
$$




$$
\begin{aligned}
& =\alpha_{n, 1}^{1}\left\|x_{n}-q\right\|^{2}+\sum_{j=2}^{\ell_{2}} \alpha_{n, j}^{1}\left(\rho^{j}\right)^{2} \prod_{i=1}^{j-1}\left(1-\alpha_{n, i}^{1}\right) \alpha_{n, 1}^{2}\left\|x_{n}-q\right\|^{2} \\
& +\prod_{i=1}^{\ell_{2}}\left(1-\alpha_{n, i}^{1}\right)\left(\rho^{j}\right)^{2} \alpha_{n, 1}^{2}\left\|x_{n}-q\right\|^{2} \\
& +\left(\sum_{j=2}^{\ell_{2}} \alpha_{n, j}^{1}\left(\rho^{j}\right)^{2} \prod_{i=1}^{j-1}\left(1-\alpha_{n, i}^{1}\right)\right)\left(\sum_{j=2}^{\ell_{3}} \alpha_{n, j}^{2}\left(\rho^{j}\right)^{2} \prod_{i=1}^{j-1}\left(1-\alpha_{n, i}^{2}\right)\right) \alpha_{n, 1}^{3}\left\|x_{n}-q\right\|^{2} \\
& +\left(\sum_{j=2}^{\ell_{2}} \alpha_{n, j}^{1}\left(\rho^{j}\right)^{2} \prod_{i=1}^{j-1}\left(1-\alpha_{n, i}^{1}\right)\right)\left(\sum_{j=2}^{\ell_{3}} \alpha_{n, j}^{2}\left(\rho^{j}\right)^{2} \prod_{i=1}^{j-1}\left(1-\alpha_{n, i}^{2}\right)\right) \\
& \times\left(\sum_{j=2}^{\ell_{4}} \alpha_{n, j}^{3}\left(\rho^{j}\right)^{2} \prod_{i=1}^{j-1}\left(1-\alpha_{n, i}^{3}\right)\right)\left\|y_{n}^{4}-q\right\|^{2}+\left(\sum_{j=2}^{\ell_{2}} \alpha_{n, j}^{1}\left(\rho^{j}\right)^{2} \prod_{i=1}^{j-1}\left(1-\alpha_{n, i}^{1}\right)\right) \\
& \times\left(\sum_{j=2}^{\ell_{3}} \alpha_{n, j}^{2}\left(\rho^{j}\right)^{2} \prod_{i=1}^{j-1}\left(1-\alpha_{n, i}^{2}\right)\right)\left(\prod_{i=1}^{\ell_{4}}\left(1-\alpha_{n, i}^{4}\right)\left(\rho^{j}\right)^{2}\right)\left\|y_{n}^{4}-q\right\|^{2} \\
& +\left(\sum_{j=2}^{\ell_{2}} \alpha_{n, j}^{1}\left(\rho^{j}\right)^{2} \prod_{i=1}^{j-1}\left(1-\alpha_{n, i}^{1}\right)\right)\left(\prod_{i=1}^{\ell_{3}}\left(1-\alpha_{n, i}^{2}\right)\left(\rho^{j}\right)^{2}\right)\left\|x_{n}-q\right\|^{2} \\
& +\left(\sum_{j=2}^{\ell_{2}} \alpha_{n, j}^{1}\left(\rho^{j}\right)^{2} \prod_{i=1}^{j-1}\left(1-\alpha_{n, i}^{1}\right)\right)\left(\prod_{i=1}^{\ell_{3}}\left(1-\alpha_{n, i}^{2}\right)\left(\rho^{j}\right)^{2}\right)\left(\sum_{j=2}^{\ell_{4}} \alpha_{n, j}^{3}\left(\rho^{j}\right)^{2} \prod_{i=1}^{j-1}\left(1-\alpha_{n, i}^{3}\right)\right)\left\|y_{n}^{4}-q\right\|^{2} \\
& +\left(\sum_{j=2}^{\ell_{2}} \alpha_{n, j}^{1}\left(\rho^{j}\right)^{2} \prod_{i=1}^{j-1}\left(1-\alpha_{n, i}^{1}\right)\right)\left(\prod_{i=1}^{\ell_{3}}\left(1-\alpha_{n, i}^{2}\right)\left(\rho^{j}\right)^{2}\right)\left(\prod_{i=1}^{\ell_{4}}\left(1-\alpha_{n, i}^{4}\right)\left(\rho^{j}\right)^{2}\right)\left\|y_{n}^{4}-q\right\|^{2} \\
& +\left(\sum_{j=2}^{\ell_{3}} \alpha_{n, j}^{2}\left(\rho^{j}\right)^{2} \prod_{i=1}^{j-1}\left(1-\alpha_{n, i}^{2}\right)\right)\left(\prod_{i=1}^{\ell_{2}}\left(1-\alpha_{n, i}^{1}\right)\left(\rho^{j}\right)^{2}\right) \alpha_{n, 1}^{3}\left\|x_{n}-q\right\|^{2} \\
& +\left(\sum_{j=2}^{\ell_{3}} \alpha_{n, j}^{2}\left(\rho^{j}\right)^{2} \prod_{i=1}^{j-1}\left(1-\alpha_{n, i}^{2}\right)\right)\left(\prod_{i=1}^{\ell_{2}}\left(1-\alpha_{n, i}^{1}\right)\left(\rho^{j}\right)^{2}\right)\left(\sum_{j=2}^{\ell_{4}} \alpha_{n, j}^{3}\left(\rho^{j}\right)^{2} \prod_{i=1}^{j-1}\left(1-\alpha_{n, i}^{3}\right)\right)\left\|y_{n}^{4}-q\right\|^{2} \\
& +\left(\sum_{j=2}^{\ell_{3}} \alpha_{n, j}^{2}\left(\rho^{j}\right)^{2} \prod_{i=1}^{j-1}\left(1-\alpha_{n, i}^{2}\right)\right)\left(\prod_{i=1}^{\ell_{2}}\left(1-\alpha_{n, i}^{1}\right)\left(\rho^{j}\right)^{2}\right)\left(\prod_{i=1}^{\ell_{4}}\left(1-\alpha_{n, i}^{4}\right)\left(\rho^{j}\right)^{2}\right)\left\|y_{n}^{4}-q\right\|^{2} \\
& +\left(\prod_{i=1}^{\ell_{3}}\left(1-\alpha_{n, i}^{2}\right)\left(\rho^{j}\right)^{2}\right)\left(\prod_{i=1}^{\ell_{2}}\left(1-\alpha_{n, i}^{1}\right)\left(\rho^{j}\right)^{2}\right) \alpha_{n, 1}^{3}\left\|x_{n}-q\right\|^{2} \\
& +\left(\prod_{i=1}^{\ell_{3}}\left(1-\alpha_{n, i}^{2}\right)\left(\rho^{j}\right)^{2}\right)\left(\prod_{i=1}^{\ell_{2}}\left(1-\alpha_{n, i}^{1}\right)\left(\rho^{j}\right)^{2}\right)\left(\sum_{j=2}^{\ell_{4}} \alpha_{n, j}^{3}\left(\rho^{j}\right)^{2} \prod_{i=1}^{j-1}\left(1-\alpha_{n, i}^{3}\right)\right)\left\|y_{n}^{4}-q\right\|^{2} \\
& +\left(\prod_{i=1}^{\ell_{3}}\left(1-\alpha_{n, i}^{2}\right)\left(\rho^{j}\right)^{2}\right)\left(\prod_{i=1}^{\ell_{2}}\left(1-\alpha_{n, i}^{1}\right)\left(\rho^{j}\right)^{2}\right)\left(\prod_{i=1}^{\ell_{4}}\left(1-\alpha_{n, i}^{4}\right)\left(\rho^{j}\right)^{2}\right)\left\|y_{n}^{4}-q\right\|^{2}
\end{aligned}
$$




$$
\begin{aligned}
& =\alpha_{n, 1}^{1}\left\|x_{n}-q\right\|^{2}+\sum_{j=2}^{\ell_{2}} \alpha_{n, j}^{1}\left(\rho^{j}\right)^{2} \prod_{i=1}^{j-1}\left(1-\alpha_{n, i}^{1}\right) \alpha_{n, 1}^{2}\left\|x_{n}-q\right\|^{2} \\
& +\prod_{i=1}^{\ell_{2}}\left(1-\alpha_{n, i}^{1}\right)\left(\rho^{j}\right)^{2} \alpha_{n, 1}^{2}\left\|x_{n}-q\right\|^{2} \\
& +\left(\sum_{j=2}^{\ell_{2}} \alpha_{n, j}^{1}\left(\rho^{j}\right)^{2} \prod_{i=1}^{j-1}\left(1-\alpha_{n, i}^{1}\right)\right)\left(\sum_{j=2}^{\ell_{3}} \alpha_{n, j}^{2}\left(\rho^{j}\right)^{2} \prod_{i=1}^{j-1}\left(1-\alpha_{n, i}^{2}\right)\right) \alpha_{n, 1}^{3}\left\|x_{n}-q\right\|^{2} \\
& +\left(\sum_{j=2}^{\ell_{2}} \alpha_{n, j}^{1}\left(\rho^{j}\right)^{2} \prod_{i=1}^{j-1}\left(1-\alpha_{n, i}^{1}\right)\right)\left(\prod_{i=1}^{\ell_{3}}\left(1-\alpha_{n, i}^{2}\right)\left(\rho^{j}\right)^{2}\right)\left\|x_{n}-q\right\|^{2} \\
& +\left(\sum_{j=2}^{\ell_{3}} \alpha_{n, j}^{2}\left(\rho^{j}\right)^{2} \prod_{i=1}^{j-1}\left(1-\alpha_{n, i}^{2}\right)\right)\left(\prod_{i=1}^{\ell_{2}}\left(1-\alpha_{n, i}^{1}\right)\left(\rho^{j}\right)^{2}\right) \alpha_{n, 1}^{3}\left\|x_{n}-q\right\|^{2} \\
& +\left(\prod_{i=1}^{\ell_{3}}\left(1-\alpha_{n, i}^{2}\right)\left(\rho^{j}\right)^{2}\right)\left(\prod_{i=1}^{\ell_{2}}\left(1-\alpha_{n, i}^{1}\right)\left(\rho^{j}\right)^{2}\right) \alpha_{n, 1}^{3}\left\|x_{n}-q\right\|^{2} \\
& +\left(\sum_{j=2}^{\ell_{2}} \alpha_{n, j}^{1}\left(\rho^{j}\right)^{2} \prod_{i=1}^{j-1}\left(1-\alpha_{n, i}^{1}\right)\right)\left(\sum_{j=2}^{\ell_{3}} \alpha_{n, j}^{2}\left(\rho^{j}\right)^{2} \prod_{i=1}^{j-1}\left(1-\alpha_{n, i}^{2}\right)\right) \\
& \times\left(\left(1-\alpha_{n, 1}^{3}-\prod_{i=1}^{\ell_{4}}\left(1-\alpha_{n, i}^{3}\right)\right)\left(\rho^{j}\right)^{2}\right)\left\|y_{n}^{4}-q\right\|^{2}+\left(\sum_{j=2}^{\ell_{2}} \alpha_{n, j}^{1}\left(\rho^{j}\right)^{2} \prod_{i=1}^{j-1}\left(1-\alpha_{n, i}^{1}\right)\right) \\
& \times\left(\sum_{j=2}^{\ell_{3}} \alpha_{n, j}^{2}\left(\rho^{j}\right)^{2} \prod_{i=1}^{j-1}\left(1-\alpha_{n, i}^{2}\right)\right)\left(\prod_{i=1}^{\ell_{4}}\left(1-\alpha_{n, i}^{4}\right)\left(\rho^{j}\right)^{2}\right)\left\|y_{n}^{4}-q\right\|^{2} \\
& +\left(\sum_{j=2}^{\ell_{2}} \alpha_{n, j}^{1}\left(\rho^{j}\right)^{2} \prod_{i=1}^{j-1}\left(1-\alpha_{n, i}^{1}\right)\right)\left(\prod_{i=1}^{\ell_{3}}\left(1-\alpha_{n, i}^{2}\right)\left(\rho^{j}\right)^{2}\right) \\
& \left.\times\left(\left(1-\alpha_{n, 1}^{3}-\prod_{i=1}^{\ell_{4}}\left(1-\alpha_{n, i}^{3}\right)\right)\left(\rho^{j}\right)^{2}\right)\right)\left\|y_{n}^{4}-q\right\|^{2} \\
& +\left(\sum_{j=2}^{\ell_{2}} \alpha_{n, j}^{1}\left(\rho^{j}\right)^{2} \prod_{i=1}^{j-1}\left(1-\alpha_{n, i}^{1}\right)\right)\left(\prod_{i=1}^{\ell_{3}}\left(1-\alpha_{n, i}^{2}\right)\left(\rho^{j}\right)^{2}\right)\left(\prod_{i=1}^{\ell_{4}}\left(1-\alpha_{n, i}^{4}\right)\left(\rho^{j}\right)^{2}\right)\left\|y_{n}^{4}-q\right\|^{2} \\
& +\left(\sum_{j=2}^{\ell_{3}} \alpha_{n, j}^{2}\left(\rho^{j}\right)^{2} \prod_{i=1}^{j-1}\left(1-\alpha_{n, i}^{2}\right)\right)\left(\prod_{i=1}^{\ell_{2}}\left(1-\alpha_{n, i}^{1}\right)\left(\rho^{j}\right)^{2}\right) \\
& \left.\times\left(\left(1-\alpha_{n, 1}^{3}-\prod_{i=1}^{\ell_{4}}\left(1-\alpha_{n, i}^{3}\right)\right)\left(\rho^{j}\right)^{2}\right)\right)\left\|y_{n}^{4}-q\right\|^{2} \\
& +\left(\sum_{j=2}^{\ell_{3}} \alpha_{n, j}^{2}\left(\rho^{j}\right)^{2} \prod_{i=1}^{j-1}\left(1-\alpha_{n, i}^{2}\right)\right)\left(\prod_{i=1}^{\ell_{2}}\left(1-\alpha_{n, i}^{1}\right)\left(\rho^{j}\right)^{2}\right)\left(\prod_{i=1}^{\ell_{4}}\left(1-\alpha_{n, i}^{4}\right)\left(\rho^{j}\right)^{2}\right)\left\|y_{n}^{4}-q\right\|^{2} \\
& +\left(\prod_{i=1}^{\ell_{3}}\left(1-\alpha_{n, i}^{2}\right)\left(\rho^{j}\right)^{2}\right)\left(\prod_{i=1}^{\ell_{2}}\left(1-\alpha_{n, i}^{1}\right)\left(\rho^{j}\right)^{2}\right)\left(\left(1-\alpha_{n, 1}^{3}-\prod_{i=1}^{\ell_{4}}\left(1-\alpha_{n, i}^{3}\right)\right)\left(\rho^{j}\right)^{2}\right)\left\|y_{n}^{4}-q\right\|^{2} \\
& +\left(\prod_{i=1}^{\ell_{3}}\left(1-\alpha_{n, i}^{2}\right)\left(\rho^{j}\right)^{2}\right)\left(\prod_{i=1}^{\ell_{2}}\left(1-\alpha_{n, i}^{1}\right)\left(\rho^{j}\right)^{2}\right)\left(\prod_{i=1}^{\ell_{4}}\left(1-\alpha_{n, i}^{4}\right)\left(\rho^{j}\right)^{2}\right)\left\|y_{n}^{4}-q\right\|^{2}
\end{aligned}
$$




$$
\begin{aligned}
& =\alpha_{n, 1}^{1}\left\|x_{n}-q\right\|^{2}+\sum_{j=2}^{\ell_{2}} \alpha_{n, j}^{1}\left(\rho^{j}\right)^{2} \prod_{i=1}^{j-1}\left(1-\alpha_{n, i}^{1}\right) \alpha_{n, 1}^{2}\left\|x_{n}-q\right\|^{2} \\
& +\prod_{i=1}^{\ell_{2}}\left(1-\alpha_{n, i}^{1}\right)\left(\rho^{j}\right)^{2} \alpha_{n, 1}^{2}\left\|x_{n}-q\right\|^{2} \\
& +\left(\sum_{j=2}^{\ell_{2}} \alpha_{n, j}^{1}\left(\rho^{j}\right)^{2} \prod_{i=1}^{j-1}\left(1-\alpha_{n, i}^{1}\right)\right)\left(\sum_{j=2}^{\ell_{3}} \alpha_{n, j}^{2}\left(\rho^{j}\right)^{2} \prod_{i=1}^{j-1}\left(1-\alpha_{n, i}^{2}\right)\right) \alpha_{n, 1}^{3}\left\|x_{n}-q\right\|^{2} \\
& +\left(\sum_{j=2}^{\ell_{2}} \alpha_{n, j}^{1}\left(\rho^{j}\right)^{2} \prod_{i=1}^{j-1}\left(1-\alpha_{n, i}^{1}\right)\right)\left(\prod_{i=1}^{\ell_{3}}\left(1-\alpha_{n, i}^{2}\right)\left(\rho^{j}\right)^{2}\right)\left\|x_{n}-q\right\|^{2} \\
& +\left(\sum_{j=2}^{\ell_{3}} \alpha_{n, j}^{2}\left(\rho^{j}\right)^{2} \prod_{i=1}^{j-1}\left(1-\alpha_{n, i}^{2}\right)\right)\left(\prod_{i=1}^{\ell_{2}}\left(1-\alpha_{n, i}^{1}\right)\left(\rho^{j}\right)^{2}\right) \alpha_{n, 1}^{3}\left\|x_{n}-q\right\|^{2} \\
& +\left(\prod_{i=1}^{\ell_{3}}\left(1-\alpha_{n, i}^{2}\right)\left(\rho^{j}\right)^{2}\right)\left(\prod_{i=1}^{\ell_{2}}\left(1-\alpha_{n, i}^{1}\right)\left(\rho^{j}\right)^{2}\right) \alpha_{n, 1}^{3}\left\|x_{n}-q\right\|^{2} \\
& +\left(\sum_{j=2}^{\ell_{2}} \alpha_{n, j}^{1}\left(\rho^{j}\right)^{2} \prod_{i=1}^{j-1}\left(1-\alpha_{n, i}^{1}\right)\right)\left(\sum_{j=2}^{\ell_{3}} \alpha_{n, j}^{2}\left(\rho^{j}\right)^{2} \prod_{i=1}^{j-1}\left(1-\alpha_{n, i}^{2}\right)\right)\left(1-\alpha_{n, 1}^{3}\right)\left(\rho^{j}\right)^{2}\left\|y_{n}^{4}-q\right\|^{2} \\
& \left.+\left(\sum_{j=2}^{\ell_{2}} \alpha_{n, j}^{1}\left(\rho^{j}\right)^{2} \prod_{i=1}^{j-1}\left(1-\alpha_{n, i}^{1}\right)\right)\left(\prod_{i=1}^{\ell_{3}}\left(1-\alpha_{n, i}^{2}\right)\left(\rho^{j}\right)^{2}\right)\left(1-\alpha_{n, 1}^{3}\right)\left(\rho^{j}\right)^{2}\right)\left\|y_{n}^{4}-q\right\|^{2} \\
& \left.+\left(\sum_{j=2}^{\ell_{3}} \alpha_{n, j}^{2}\left(\rho^{j}\right)^{2} \prod_{i=1}^{j-1}\left(1-\alpha_{n, i}^{2}\right)\right)\left(\prod_{i=1}^{\ell_{2}}\left(1-\alpha_{n, i}^{1}\right)\left(\rho^{j}\right)^{2}\right)\left(1-\alpha_{n, 1}^{3}\right)\left(\rho^{j}\right)^{2}\right)\left\|y_{n}^{4}-q\right\|^{2} \\
& +\left(\prod_{i=1}^{\ell_{3}}\left(1-\alpha_{n, i}^{2}\right)\left(\rho^{j}\right)^{2}\right)\left(\prod_{i=1}^{\ell_{2}}\left(1-\alpha_{n, i}^{1}\right)\left(\rho^{j}\right)^{2}\right)\left(1-\alpha_{n, 1}^{3}\right)\left(\rho^{j}\right)^{2}\left\|y_{n}^{4}-q\right\|^{2} \\
& \leq \alpha_{n, 1}^{1}\left\|x_{n}-q\right\|^{2}+\sum_{j=2}^{\ell_{2}} \alpha_{n, j}^{1}\left(\rho^{j}\right)^{2} \prod_{i=1}^{j-1}\left(1-\alpha_{n, i}^{1}\right) \alpha_{n, 1}^{2}\left\|x_{n}-q\right\|^{2} \\
& +\prod_{i=1}^{\ell_{2}}\left(1-\alpha_{n, i}^{1}\right)\left(\rho^{j}\right)^{2} \alpha_{n, 1}^{2}\left\|x_{n}-q\right\|^{2} \\
& +\left(\sum_{j=2}^{\ell_{2}} \alpha_{n, j}^{1}\left(\rho^{j}\right)^{2} \prod_{i=1}^{j-1}\left(1-\alpha_{n, i}^{1}\right)\right)\left(\sum_{j=2}^{\ell_{3}} \alpha_{n, j}^{2}\left(\rho^{j}\right)^{2} \prod_{i=1}^{j-1}\left(1-\alpha_{n, i}^{2}\right)\right) \alpha_{n, 1}^{3}\left\|x_{n}-q\right\|^{2} \\
& +\left(\sum_{j=2}^{\ell_{2}} \alpha_{n, j}^{1}\left(\rho^{j}\right)^{2} \prod_{i=1}^{j-1}\left(1-\alpha_{n, i}^{1}\right)\right)\left(\prod_{i=1}^{\ell_{3}}\left(1-\alpha_{n, i}^{2}\right)\left(\rho^{j}\right)^{2}\right)\left\|x_{n}-q\right\|^{2} \\
& +\left(\sum_{j=2}^{\ell_{3}} \alpha_{n, j}^{2}\left(\rho^{j}\right)^{2} \prod_{i=1}^{j-1}\left(1-\alpha_{n, i}^{2}\right)\right)\left(\prod_{i=1}^{\ell_{2}}\left(1-\alpha_{n, i}^{1}\right)\left(\rho^{j}\right)^{2}\right) \alpha_{n, 1}^{3}\left\|x_{n}-q\right\|^{2}
\end{aligned}
$$




$$
\begin{aligned}
& +\left(\prod_{i=1}^{\ell_{3}}\left(1-\alpha_{n, i}^{2}\right)\left(\rho^{j}\right)^{2}\right)\left(\prod_{i=1}^{\ell_{2}}\left(1-\alpha_{n, i}^{1}\right)\left(\rho^{j}\right)^{2}\right) \alpha_{n, 1}^{3}\left\|x_{n}-q\right\|^{2} \\
& +\left(\sum_{j=2}^{\ell_{2}} \alpha_{n, j}^{1}\left(\rho^{j}\right)^{2} \prod_{i=1}^{j-1}\left(1-\alpha_{n, i}^{1}\right)\right)\left(\sum_{j=2}^{\ell_{3}} \alpha_{n, j}^{2}\left(\rho^{j}\right)^{2} \prod_{i=1}^{j-1}\left(1-\alpha_{n, i}^{2}\right)\right) \alpha_{n, 1}^{3}\left(1-\alpha_{n, 1}^{3}\right)\left(\rho^{j}\right)^{2}\left\|x_{n}-q\right\|^{2} \\
& \left.+\left(\sum_{j=2}^{\ell_{2}} \alpha_{n, j}^{1}\left(\rho^{j}\right)^{2} \prod_{i=1}^{j-1}\left(1-\alpha_{n, i}^{1}\right)\right)\left(\prod_{i=1}^{\ell_{3}}\left(1-\alpha_{n, i}^{2}\right)\left(\rho^{j}\right)^{2}\right) \alpha_{n, 1}^{3}\left(1-\alpha_{n, 1}^{3}\right)\left(\rho^{j}\right)^{2}\right)\left\|x_{n}-q\right\|^{2} \\
& \left.+\left(\sum_{j=2}^{\ell_{3}} \alpha_{n, j}^{2}\left(\rho^{j}\right)^{2} \prod_{i=1}^{j-1}\left(1-\alpha_{n, i}^{2}\right)\right)\left(\prod_{i=1}^{\ell_{2}}\left(1-\alpha_{n, i}^{1}\right)\left(\rho^{j}\right)^{2}\right) \alpha_{n, 1}^{3}\left(1-\alpha_{n, 1}^{3}\right)\left(\rho^{j}\right)^{2}\right)\left\|x_{n}-q\right\|^{2} \\
& +\left(\prod_{i=1}^{\ell_{3}}\left(1-\alpha_{n, i}^{2}\right)\left(\rho^{j}\right)^{2}\right)\left(\prod_{i=1}^{\ell_{2}}\left(1-\alpha_{n, i}^{1}\right)\left(\rho^{j}\right)^{2}\right) \alpha_{n, 1}^{3}\left(1-\alpha_{n, 1}^{3}\right)\left(\rho^{j}\right)^{2}\left\|x_{n}-q\right\|^{2}+\cdots \\
& +\left(\sum_{j=2}^{\ell_{2}} \alpha_{n, j}^{1}\left(\rho^{j}\right)^{2} \prod_{i=1}^{j-1}\left(1-\alpha_{n, i}^{1}\right)\right)\left(\sum_{j=2}^{\ell_{3}} \alpha_{n, j}^{2}\left(\rho^{j}\right)^{2} \prod_{i=1}^{j-1}\left(1-\alpha_{n, i}^{2}\right)\right) \\
& \times\left(\sum_{j=2}^{\ell_{4}} \alpha_{n, j}^{3}\left(\rho^{j}\right)^{3} \prod_{i=1}^{j-1}\left(1-\alpha_{n, i}^{2}\right)\right) \times \cdots \times\left(\sum_{j=2}^{\ell_{s-1}} \alpha_{n, j}^{\ell_{s-2}}\left(\rho^{j}\right)^{2} \prod_{i=1}^{j-1}\left(1-\alpha_{n, i}^{\ell_{s-2}}\right)\right) \\
& \times\left(\sum_{j=2}^{\ell_{s}} \alpha_{n, j}^{\ell_{s-1}}\left(\rho^{j}\right)^{2} \prod_{i=1}^{j-1}\left(1-\alpha_{n, i}^{\ell_{s-1}}\right)\right) \alpha_{n, 1}^{s}\left\|x_{n}-q\right\|^{2}+\left(\rho^{j}\right)^{2}\left(\prod_{i=1}^{\ell_{2}}\left(1-\alpha_{n, i}^{1}\right)\left(\rho^{j}\right)^{2}\right) \\
& \times\left(\rho^{j}\right)^{2}\left(\prod_{i=1}^{\ell_{3}}\left(1-\alpha_{n, i}^{2}\right)\left(\rho^{j}\right)^{2}\right)\left(\rho^{j}\right)^{2}\left(\prod_{i=1}^{\ell_{4}}\left(1-\alpha_{n, i}^{3}\right)\left(\rho^{j}\right)^{2}\right) \times \cdots \times\left(\rho^{j}\right)^{2}\left(\prod_{i=1}^{\ell_{s-1}}\left(1-\alpha_{n, i}^{\ell_{s-2}}\right)\left(\rho^{j}\right)^{2}\right) \\
& \times\left(\rho^{j}\right)^{2}\left(\prod_{i=1}^{\ell_{s}}\left(1-\alpha_{n, i}^{\ell_{s-1}}\right)\left(\rho^{j}\right)^{2}\right)\left\|x_{n}-q\right\|^{2} \\
& <\alpha_{n, 1}^{1}\left\|x_{n}-q\right\|^{2}+\sum_{j=2}^{\ell_{2}} \alpha_{n, j}^{1} \prod_{i=1}^{j-1}\left(1-\alpha_{n, i}^{1}\right) \alpha_{n, 1}^{2}\left\|x_{n}-q\right\|^{2} \\
& +\prod_{i=1}^{\ell_{2}}\left(1-\alpha_{n, i}^{1}\right) \alpha_{n, 1}^{2}\left\|x_{n}-q\right\|^{2} \\
& +\left(\sum_{j=2}^{\ell_{2}} \alpha_{n, j}^{1} \prod_{i=1}^{j-1}\left(1-\alpha_{n, i}^{1}\right)\right)\left(1-\alpha_{n, 1}^{2}-\prod_{i=1}^{\ell_{3}}\left(1-\alpha_{n, i}^{2}\right)\right) \alpha_{n, 1}^{3}\left\|x_{n}-q\right\|^{2} \\
& +\left(\sum_{j=2}^{\ell_{2}} \alpha_{n, j}^{1} \prod_{i=1}^{j-1}\left(1-\alpha_{n, i}^{1}\right)\right)\left(\prod_{i=1}^{\ell_{3}}\left(1-\alpha_{n, i}^{2}\right)\right)\left\|x_{n}-q\right\|^{2} \\
& +\left(1-\alpha_{n, 1}^{2}-\prod_{i=1}^{\ell_{3}}\left(1-\alpha_{n, i}^{2}\right)\right)\left(\prod_{i=1}^{\ell_{2}}\left(1-\alpha_{n, i}^{1}\right)\right) \alpha_{n, 1}^{3}\left\|x_{n}-q\right\|^{2} \\
& +\left(\prod_{i=1}^{\ell_{3}}\left(1-\alpha_{n, i}^{2}\right)\right)\left(\prod_{i=1}^{\ell_{2}}\left(1-\alpha_{n, i}^{1}\right)\right) \alpha_{n, 1}^{3}\left\|x_{n}-q\right\|^{2}
\end{aligned}
$$




$$
\begin{aligned}
& +\left(\sum_{j=2}^{\ell_{2}} \alpha_{n, j}^{1} \prod_{i=1}^{j-1}\left(1-\alpha_{n, i}^{1}\right)\right)\left(1-\alpha_{n, 1}^{2}-\prod_{i=1}^{\ell_{3}}\left(1-\alpha_{n, i}^{2}\right)\right) \alpha_{n, 1}^{3}\left(1-\alpha_{n, 1}^{3}\right)\left\|x_{n}-q\right\|^{2} \\
& +\left(\sum_{j=2}^{\ell_{2}} \alpha_{n, j}^{1} \prod_{i=1}^{j-1}\left(1-\alpha_{n, i}^{1}\right)\right)\left(\prod_{i=1}^{\ell_{3}}\left(1-\alpha_{n, i}^{2}\right)\right) \alpha_{n, 1}^{3}\left(1-\alpha_{n, 1}^{3}\right)\left\|x_{n}-q\right\|^{2} \\
& \left.+\left(1-\alpha_{n, 1}^{2}-\prod_{i=1}^{\ell_{3}}\left(1-\alpha_{n, i}^{2}\right)\right)\right)\left(\prod_{i=1}^{\ell_{2}}\left(1-\alpha_{n, i}^{1}\right)\right) \alpha_{n, 1}^{3}\left(1-\alpha_{n, 1}^{3}\right)\left\|x_{n}-q\right\|^{2} \\
& +\left(\prod_{i=1}^{\ell_{3}}\left(1-\alpha_{n, i}^{2}\right)\right)\left(\prod_{i=1}^{\ell_{2}}\left(1-\alpha_{n, i}^{1}\right)\right) \alpha_{n, 1}^{3}\left(1-\alpha_{n, 1}^{3}\right)\left\|x_{n}-q\right\|^{2}+\cdots \\
& +\left(1-\alpha_{n, 1}^{1}-\prod_{i=1}^{\ell_{2}}\left(1-\alpha_{n, i}^{2}\right)\right)\left(1-\alpha_{n, 1}^{2}-\prod_{i=1}^{\ell_{3}}\left(1-\alpha_{n, i}^{2}\right)\right) \\
& \times\left(1-\alpha_{n, 1}^{3}-\prod_{i=1}^{\ell_{4}}\left(1-\alpha_{n, i}^{3}\right)\right) \times \cdots \times\left(1-\alpha_{n, 1}^{\ell_{s-2}}-\prod_{i=1}^{\ell_{s-1}}\left(1-\alpha_{n, i}^{\ell_{s-2}}\right)\right) \\
& \times\left(1-\alpha_{n, 1}^{\ell_{s-1}}-\prod_{i=1}^{\ell_{s}}\left(1-\alpha_{n, i}^{\ell_{s-1}}\right)\right) \alpha_{n, 1}^{s}\left\|x_{n}-q\right\|^{2}+\left(\prod_{i=1}^{\ell_{2}}\left(1-\alpha_{n, i}^{1}\right)\right) \\
& \times\left(\prod_{i=1}^{\ell_{3}}\left(1-\alpha_{n, i}^{2}\right)\right)\left(\prod_{i=1}^{\ell_{4}}\left(1-\alpha_{n, i}^{3}\right)\right) \times \cdots \times\left(\prod_{i=1}^{\ell_{s-1}}\left(1-\alpha_{n, i}^{\ell_{s-2}}\right)\right) \\
& \times\left(\prod_{i=1}^{\ell_{s}}\left(1-\alpha_{n, i}^{\ell_{s-1}}\right)\right)\left\|x_{n}-q\right\|^{2} \\
& <\alpha_{n, 1}^{1}\left\|x_{n}-q\right\|^{2}+\sum_{j=2}^{\ell_{2}} \alpha_{n, j}^{1} \prod_{i=1}^{j-1}\left(1-\alpha_{n, i}^{1}\right) \alpha_{n, 1}^{2}\left\|x_{n}-q\right\|^{2} \\
& +\prod_{i=1}^{\ell_{2}}\left(1-\alpha_{n, i}^{1}\right) \alpha_{n, 1}^{2}\left\|x_{n}-q\right\|^{2} \\
& +\left(\sum_{j=2}^{\ell_{2}} \alpha_{n, j}^{1} \prod_{i=1}^{j-1}\left(1-\alpha_{n, i}^{1}\right)\right) \alpha_{n, 1}^{3}\left(1-\alpha_{n, 1}^{2}\right)\left\|x_{n}-q\right\|^{2} \\
& +\alpha_{n, 1}^{3}\left(1-\alpha_{n, 1}^{2}\right)\left(\prod_{i=1}^{\ell_{2}}\left(1-\alpha_{n, i}^{1}\right)\right)\left\|x_{n}-q\right\|^{2} \\
& +\left(\sum_{j=2}^{\ell_{2}} \alpha_{n, j}^{1} \prod_{i=1}^{j-1}\left(1-\alpha_{n, i}^{1}\right)\right)\left(1-\alpha_{n, 1}^{2}\right) \alpha_{n, 1}^{3}\left(1-\alpha_{n, 1}^{3}\right)\left\|x_{n}-q\right\|^{2} \\
& +\left(1-\alpha_{n, 1}^{2}\right)\left(\prod_{i=1}^{\ell_{2}}\left(1-\alpha_{n, i}^{1}\right)\right) \alpha_{n, 1}^{3}\left(1-\alpha_{n, 1}^{3}\right)\left\|x_{n}-q\right\|^{2}+\cdots+ \\
& +\left(1-\alpha_{n, 1}^{1}\right)\left(1-\alpha_{n, 1}^{2}\right)\left(1-\alpha_{n, 1}^{3}\right) \times \cdots \times\left(1-\alpha_{n, 1}^{\ell_{s-2}}\right) \\
& \times\left(1-\alpha_{n, 1}^{\ell_{s-1}}\right) \alpha_{n, 1}^{s}\left\|x_{n}-q\right\|^{2}
\end{aligned}
$$




$$
\begin{aligned}
= & \alpha_{n, 1}^{1}\left\|x_{n}-q\right\|^{2}+\alpha_{n, 1}^{2}\left(1-\alpha_{n, 1}^{1}-\prod_{i=1}^{\ell_{2}}\left(1-\alpha_{n, i}^{1}\right)\right)\left\|x_{n}-q\right\|^{2} \\
& +\prod_{i=1}^{\ell_{2}}\left(1-\alpha_{n, i}^{1}\right) \alpha_{n, 1}^{2}\left\|x_{n}-q\right\|^{2} \\
& +\left(\left(1-\alpha_{n, 1}^{1}-\prod_{i=1}^{\ell_{2}}\left(1-\alpha_{n, i}^{1}\right)\right) \alpha_{n, 1}^{3}\left(1-\alpha_{n, 1}^{2}\right)\left\|x_{n}-q\right\|^{2}\right. \\
& +\alpha_{n, 1}^{3}\left(1-\alpha_{n, 1}^{2}\right)\left(\prod_{i=1}^{\ell_{2}}\left(1-\alpha_{n, i}^{1}\right)\right)\left\|x_{n}-q\right\|^{2} \\
& +\left(\left(1-\alpha_{n, 1}^{1}-\prod_{i=1}^{\ell_{2}}\left(1-\alpha_{n, i}^{1}\right)\right)\left(1-\alpha_{n, 1}^{2}\right) \alpha_{n, 1}^{3}\left(1-\alpha_{n, 1}^{3}\right)\left\|x_{n}-q\right\|^{2}\right. \\
& +\left(1-\alpha_{n, 1}^{2}\right)\left(\prod_{i=1}^{\ell_{2}}\left(1-\alpha_{n, i}^{1}\right)\right) \alpha_{n, 1}^{3}\left(1-\alpha_{n, 1}^{3}\right)\left\|x_{n}-q\right\|^{2}+\cdots+ \\
& +\left(1-\alpha_{n, 1}^{1}\right)\left(1-\alpha_{n, 1}^{2}\right)\left(1-\alpha_{n, 1}^{3}\right) \times \cdots \times\left(1-\alpha_{n, 1}^{\ell_{s-2}}\right) \\
& \times\left(1-\alpha_{n, 1}^{\ell_{s-1}}\right) \alpha_{n, 1}^{s}\left\|x_{n}-q\right\|^{2} \\
< & {\left[\alpha_{n, 1}^{1}+\alpha_{n, 1}^{2}\left(1-\alpha_{n, 1}^{1}\right)+\left(1-\alpha_{n, 1}^{1}\right) \alpha_{n, 1}^{3}\left(1-\alpha_{n, 1}^{2}\right)+\left(1-\alpha_{n, 1}^{1}\right)\left(1-\alpha_{n, 1}^{2}\right) \alpha_{n, 1}^{3}\left(1-\alpha_{n, 1}^{3}\right)\right.} \\
& +\cdots+\left(1-\alpha_{n, 1}^{1}\right)\left(1-\alpha_{n, 1}^{2}\right)\left(1-\alpha_{n, 1}^{3}\right) \times \cdots \times\left(1-\alpha_{n, 1}^{\ell_{s-2}}\right) \\
& \left.\times\left(1-\alpha_{n, 1}^{\ell_{s-1}}\right)\right]\left\|x_{n}-q\right\|^{2}
\end{aligned}
$$

(3.6) and (3.8) imply that

$$
\begin{aligned}
\left\|x_{n+1}-q\right\|^{2} \leq & \left\{\delta_{n, 1}+\left(1-\delta_{n, 1}\right)\left[\alpha_{n, 1}^{1}+\alpha_{n, 1}^{2}\left(1-\alpha_{n, 1}^{1}\right)+\left(1-\alpha_{n, 1}^{1}\right) \alpha_{n, 1}^{3}\left(1-\alpha_{n, 1}^{2}\right)\right.\right. \\
& +\left(1-\alpha_{n, 1}^{1}\right)\left(1-\alpha_{n, 1}^{2}\right)\left(1-\alpha_{n, 1}^{3}\right)+\cdots+\left(1-\alpha_{n, 1}^{1}\right)\left(1-\alpha_{n, 1}^{2}\right)\left(1-\alpha_{n, 1}^{3}\right) \\
& \left.\left.\times \cdots \times\left(1-\alpha_{n, 1}^{\ell_{s-2}}\right) \times\left(1-\alpha_{n, 1}^{\ell_{s-1}}\right)\right]\right\}\left\|x_{n}-q\right\|^{2}
\end{aligned}
$$

Using Lemma 2.3, we obtain (from (3.9)) that the sequence $\left\{x_{n}\right\}_{n=0}^{\infty}$ converges strongly to $q \in F(\Gamma)$; and this completes the proof.

Theorem 3.2. Let $H$ be a Hilbert space, $\Gamma: H \longrightarrow H$ be a self-map of $H$ satisfying the contractive condition

$$
\left\|\Gamma^{j} x-\Gamma^{j} y\right\| \leq \rho^{j}\|x-y\|+\sum_{i=0}^{j}\left(\begin{array}{c}
j \\
i
\end{array}\right) \rho^{j-1} \phi(\|x-\Gamma x\|),
$$

where $x, y \in H, 0 \leq \rho^{j}<1$, and let $\phi$ retain its usual meaning with $\phi(0)=0$ and $\phi(M t)=$ $M \phi(t), M \geq 0, t \in \mathbb{R}^{+}$. For arbitrary $x_{0} \in H$, let $\left\{\omega_{n}\right\}_{n=0}^{\infty}$ be the multistep DI-iteration scheme defined by (3.2). Then,

(i) $\Gamma$ defined by (3.10) has a fixed point $q$; 
(ii) the multistep SH-iteration scheme converges strongly tp $q \in \Gamma$.

Proof. We first show that $\Gamma$ satisfying condition of (3.10) has a fixed point. Assume there exists two points $q_{1}, q_{2} \in F(\Gamma)$ with $0<\left\|q_{1}-q_{2}\right\|$. Then, we have

$$
\begin{aligned}
0<\left\|q_{1}-q_{2}\right\|=\left\|\Gamma^{j} q_{1}-\Gamma^{j} q_{2}\right\| & \leq \rho^{j}\left\|q_{1}-q_{2}\right\|+\sum_{i=0}^{j}\left(\begin{array}{c}
j \\
i
\end{array}\right) \rho^{j-1} \phi\left(\left\|q \sqrt{1}-\Gamma q_{1}\right\|\right) \\
& =\rho^{j}\left\|q_{1}-q_{2}\right\|+\sum_{i=0}^{j}\left(\begin{array}{c}
j \\
i
\end{array}\right) \rho^{j-1} \phi(0)
\end{aligned}
$$

$\Rightarrow\left(1-\rho^{j}\right) \rho^{j}\left\|q_{1}-q_{2}\right\| \leq 0$. Using the fact that $\rho^{j} \in\left[[0,1)\right.$, we get $0<1-\rho^{j}$ and $\left\|q_{1}-q_{2}\right\| \leq 0$. Since the norm is a nonnegative function, we get $\left\|q_{1}-q_{2}\right\|=0 ; q_{1}=q_{2}=q($ say). Therefore, $\Gamma$ converges uniquely to a point of $F(\Gamma)$.

Now, we show that the sequence defined by (3.1) converges strongly to $q \in F(\Gamma)$. Using (3.2) and Proposition 2.4 with $x_{n+1}=y, u=q, y_{n}^{1}=t, j=i, k=1, \Gamma^{j-1} y_{n}^{1}=v_{j-1}$ and $\Gamma^{\ell_{1}} y_{n}^{1}=v$, we get

$$
\begin{aligned}
\left\|x_{n+1}-q\right\|^{2}= & \delta_{n, 1}\left\|y_{n}^{1}-q\right\|^{2}+\sum_{j=2}^{\ell_{1}} \delta_{n, j} \prod_{i=1}^{j-1}\left(1-\delta_{n, i}\right)\left\|\Gamma^{j-1} y_{n}^{1}-\Gamma^{j-1} q\right\|^{2} \\
& +\prod_{i=1}^{\ell_{1}}\left(1-\delta_{n, i}\right)\left\|\Gamma^{\ell_{1}} y_{n}^{1}-\Gamma^{\ell_{1}} q\right\|^{2}
\end{aligned}
$$

But from (3.10), with $y=y_{n}^{1}$, we have

$$
\begin{aligned}
\left\|\Gamma^{j-1} y_{n}^{1}-\Gamma^{j-1} q\right\| & \leq \rho^{j}\left\|y_{n}^{1}-q\right\|+\sum_{i=0}^{j}\left(\begin{array}{c}
j \\
i
\end{array}\right) \rho^{j-1} \phi(\|q-\Gamma q\|) \\
& =\rho^{j}\left\|y_{n}^{1}-q\right\|
\end{aligned}
$$

Proposition 2.3, (3.11) and (3.12) imply

$$
\begin{aligned}
\left\|x_{n+1}-q\right\|^{2} \leq & \delta_{n, 1}^{1}\left\|y_{n}^{1}-q\right\|^{2}+\sum_{j=2}^{\ell_{1}} \delta_{n, j}\left(\rho^{j}\right)^{2} \prod_{i=1}^{j-1}\left(1-\delta_{n, i}\right)\left\|y_{n}^{1}-q\right\|^{2} \\
& +\prod_{i=1}^{\ell_{1}}\left(1-\delta_{n, i}\right)\left(\rho^{j}\right)^{2}\left\|y_{n}^{1}-q\right\|^{2} \\
= & \delta_{n, 1}^{1}\left\|y_{n}^{1}-q\right\|^{2}+\left(1-\delta_{n, 1}^{1}-\prod_{i=1}^{\ell_{1}}\left(1-\delta_{n, i}\right)\left(\rho^{j}\right)^{2}\right)\left\|y_{n}^{1}-q\right\|^{2} \\
& +\prod_{i=1}^{\ell_{1}}\left(1-\delta_{n, i}\right)\left(\rho^{j}\right)^{2}\left\|y_{n}^{1}-q\right\|^{2} \\
= & \left\|y_{n}^{1}-q\right\|^{2}
\end{aligned}
$$


Since $\ell_{1}, \ell_{k}$ are fixed integers and $\alpha_{n, i}^{s} \in[0,1]$ for each $s$, we have (using Proposition 2.3, (3.2) and (3.12)) the following estimates for $n=1,2, \cdots$ and $1 \leq s \leq k-1$ :

$$
\begin{aligned}
& \left\|y_{n}^{1}-q\right\|^{2} \leq \alpha_{n, 1}^{1}\left\|y_{n}^{2}-q\right\|^{2}+\sum_{j=2}^{\ell_{2}} \alpha_{n, j}^{1} \prod_{i=1}^{j-1}\left(1-\alpha_{n, i}^{1}\right)\left\|\Gamma^{j-1} y_{n}^{2}-\Gamma^{j-1} q\right\|^{2} \\
& +\prod_{i=1}^{\ell_{2}}\left(1-\alpha_{n, i}^{1}\right)\left\|\Gamma^{\ell_{2}} y_{n}^{2}-\Gamma^{\ell_{2}} q\right\|^{2} \\
& \leq\left(\alpha_{n, 1}^{1}+\sum_{j=2}^{\ell_{2}} \alpha_{n, j}^{1}\left(\rho^{j}\right)^{2} \prod_{i=1}^{j-1}\left(1-\alpha_{n, i}^{1}\right)+\prod_{i=1}^{\ell_{2}}\left(1-\alpha_{n, i}^{1}\right)\left(\rho^{j}\right)^{2}\right)\left\|y_{n}^{2}-q\right\|^{2} \\
& \leq\left(\alpha_{n, 1}^{1}+\sum_{j=2}^{\ell_{2}} \alpha_{n, j}^{2}\left(\rho^{j}\right)^{2} \prod_{i=1}^{j-1}\left(1-\alpha_{n, i}^{1}\right)+\prod_{i=1}^{\ell_{2}}\left(1-\alpha_{n, i}^{1}\right)\left(\rho^{j}\right)^{2}\right)\left[\alpha_{n, 1}^{2}\left\|y_{n}^{3}-q\right\|^{2}\right. \\
& \left.+\sum_{j=2}^{\ell_{3}} \alpha_{n, j}^{2} \prod_{i=1}^{j-1}\left(1-\alpha_{n, i}^{2}\right)\left\|\Gamma^{j-1} y_{n}^{3}-\Gamma^{j-1} q\right\|^{2}+\prod_{i=1}^{\ell_{3}}\left(1-\alpha_{n, i}^{2}\right)\left\|\Gamma^{\ell_{3}} y_{n}^{3}-\Gamma^{\ell_{3}} q\right\|^{2}\right] \\
& \leq\left(\alpha_{n, 1}^{1}+\sum_{j=2}^{\ell_{2}} \alpha_{n, j}^{1}\left(\rho^{j}\right)^{2} \prod_{i=1}^{j-1}\left(1-\alpha_{n, i}^{1}\right)+\prod_{i=1}^{\ell_{2}}\left(1-\alpha_{n, i}^{1}\right)\left(\rho^{j}\right)^{2}\right)\left[\alpha_{n, 1}^{2}\left\|y_{n}^{3}-q\right\|^{2}\right. \\
& \left.+\sum_{j=2}^{\ell_{3}} \alpha_{n, j}^{2}\left(\rho^{j}\right)^{2} \prod_{i=1}^{j-1}\left(1-\alpha_{n, i}\right)\left\|y_{n}^{2}-q\right\|^{2}+\prod_{i=1}^{\ell_{3}}\left(1-\alpha_{n, i}^{2}\right)\left(\rho^{j}\right)^{2}\left\|y_{n}^{3}-q\right\|^{2}\right] \\
& =\left(\alpha_{n, 1}^{1}+\sum_{j=2}^{\ell_{2}} \alpha_{n, j}^{1}\left(\rho^{j}\right)^{2} \prod_{i=1}^{j-1}\left(1-\alpha_{n, i}^{1}\right)+\prod_{i=1}^{\ell_{2}}\left(1-\alpha_{n, i}^{1}\right)\left(\rho^{j}\right)^{2}\right) \\
& \times\left(\alpha_{n, 1}^{2}+\sum_{j=2}^{\ell_{3}} \alpha_{n, j}^{2}\left(\rho^{j}\right)^{2} \prod_{i=1}^{j-1}\left(1-\alpha_{n, i}\right)+\prod_{i=1}^{\ell_{3}}\left(1-\alpha_{n, i}^{2}\right)\left(\rho^{j}\right)^{2}\right)\left\|y_{n}^{3}-q\right\|^{2} \\
& \leq\left(\alpha_{n, 1}^{1}+\sum_{j=2}^{\ell_{2}} \alpha_{n, j}^{1}\left(\rho^{j}\right)^{2} \prod_{i=1}^{j-1}\left(1-\alpha_{n, i}^{1}\right)+\prod_{i=1}^{\ell_{2}}\left(1-\alpha_{n, i}^{1}\right)\left(\rho^{j}\right)^{2}\right) \\
& \times\left(\alpha_{n, 1}^{2}+\sum_{j=2}^{\ell_{3}} \alpha_{n, j}^{2}\left(\rho^{j}\right)^{2} \prod_{i=1}^{j-1}\left(1-\alpha_{n, i}\right)+\prod_{i=1}^{\ell_{3}}\left(1-\alpha_{n, i}^{2}\right)\left(\rho^{j}\right)^{2}\right)\left[\alpha_{n, 1}^{3}\left\|y_{n}^{4}-q\right\|^{2}\right. \\
& \left.+\sum_{j=2}^{\ell_{4}} \alpha_{n, j}^{3} \prod_{i=1}^{j-1}\left(1-\alpha_{n, i}^{3}\right)\left\|\Gamma^{j-1} y_{n}^{4}-\Gamma^{j-1} q\right\|^{2}+\prod_{i=1}^{\ell_{4}}\left(1-\alpha_{n, i}^{3}\right)\left\|\Gamma^{\ell_{4}} y_{n}^{4}-\Gamma^{\ell_{4}} q\right\|^{2}\right] \\
& \leq\left(\alpha_{n, 1}^{1}+\sum_{j=2}^{\ell_{2}} \alpha_{n, j}^{1}\left(\rho^{j}\right)^{2} \prod_{i=1}^{j-1}\left(1-\alpha_{n, i}^{1}\right)+\prod_{i=1}^{\ell_{2}}\left(1-\alpha_{n, i}^{1}\right)\left(\rho^{j}\right)^{2}\right)
\end{aligned}
$$




$$
\begin{aligned}
& \times\left(\alpha_{n, 1}^{2}+\sum_{j=2}^{\ell_{3}} \alpha_{n, j}^{2}\left(\rho^{j}\right)^{2} \prod_{i=1}^{j-1}\left(1-\alpha_{n, i}\right)+\prod_{i=1}^{\ell_{3}}\left(1-\alpha_{n, i}^{2}\right)\left(\rho^{j}\right)^{2}\right)\left[\alpha_{n, 1}^{3}\left\|y_{n}^{4}-q\right\|^{2}\right. \\
& \left.+\sum_{j=2}^{\ell_{4}} \alpha_{n, j}^{3}\left(\rho^{j}\right)^{2} \prod_{i=1}^{j-1}\left(1-\alpha_{n, i}^{3}\right)\left\|y_{n}^{4}-q\right\|^{2}+\prod_{i=1}^{\ell_{4}}\left(1-\alpha_{n, i}^{3}\right)\left(\rho^{j}\right)^{2}\left\|y_{n}^{4}-q\right\|^{2}\right] \\
& =\left(\alpha_{n, 1}^{1}+\sum_{j=2}^{\ell_{2}} \alpha_{n, j}^{1}\left(\rho^{j}\right)^{2} \prod_{i=1}^{j-1}\left(1-\alpha_{n, i}^{1}\right)+\prod_{i=1}^{\ell_{2}}\left(1-\alpha_{n, i}^{1}\right)\left(\rho^{j}\right)^{2}\right) \\
& \times\left(\alpha_{n, 1}^{2}+\sum_{j=2}^{\ell_{3}} \alpha_{n, j}^{2}\left(\rho^{j}\right)^{2} \prod_{i=1}^{j-1}\left(1-\alpha_{n, i}\right)+\prod_{i=1}^{\ell_{3}}\left(1-\alpha_{n, i}^{2}\right)\left(\rho^{j}\right)^{2}\right) \\
& \times\left(\alpha_{n, 1}^{3}+\sum_{j=2}^{\ell_{4}} \alpha_{n, j}^{3}\left(\rho^{j}\right)^{2} \prod_{i=1}^{j-1}\left(1-\alpha_{n, i}^{3}\right)+\prod_{i=1}^{\ell_{4}}\left(1-\alpha_{n, i}^{3}\right)\left(\rho^{j}\right)^{2}\right)\left\|y_{n}^{4}-q\right\|^{2} \\
& \leq\left(\alpha_{n, 1}^{1}+\sum_{j=2}^{\ell_{2}} \alpha_{n, j}^{1}\left(\rho^{j}\right)^{2} \prod_{i=1}^{j-1}\left(1-\alpha_{n, i}^{1}\right)+\prod_{i=1}^{\ell_{2}}\left(1-\alpha_{n, i}^{1}\right)\left(\rho^{j}\right)^{2}\right) \\
& \times\left(\alpha_{n, 1}^{2}+\sum_{j=2}^{\ell_{3}} \alpha_{n, j}^{2}\left(\rho^{j}\right)^{2} \prod_{i=1}^{j-1}\left(1-\alpha_{n, i}\right)+\prod_{i=1}^{\ell_{3}}\left(1-\alpha_{n, i}^{2}\right)\left(\rho^{j}\right)^{2}\right) \\
& \times\left(\alpha_{n, 1}^{3}+\sum_{j=2}^{\ell_{4}} \alpha_{n, j}^{3}\left(\rho^{j}\right)^{2} \prod_{i=1}^{j-1}\left(1-\alpha_{n, i}^{3}\right)+\prod_{i=1}^{\ell_{4}}\left(1-\alpha_{n, i}^{3}\right)\left(\rho^{j}\right)^{2}\right)\left[\alpha_{n, 1}^{4}\left\|y_{n}^{5}-q\right\|^{2}\right. \\
& \left.+\sum_{j=2}^{\ell_{5}} \alpha_{n, j}^{4} \prod_{i=1}^{j-1}\left(1-\alpha_{n, i}^{4}\right)\left\|\Gamma^{j-1} y_{n}^{5}-\Gamma^{j-1} q\right\|^{2}+\prod_{i=1}^{\ell_{5}}\left(1-\alpha_{n, i}^{4}\right)\left\|\Gamma^{\ell_{5}} y_{n}^{5}-\Gamma^{\ell_{5}} q\right\|^{2}\right] \\
& \leq\left(\alpha_{n, 1}^{1}+\sum_{j=2}^{\ell_{2}} \alpha_{n, j}^{1}\left(\rho^{j}\right)^{2} \prod_{i=1}^{j-1}\left(1-\alpha_{n, i}^{1}\right)+\prod_{i=1}^{\ell_{2}}\left(1-\alpha_{n, i}^{1}\right)\left(\rho^{j}\right)^{2}\right) \\
& \times\left(\alpha_{n, 1}^{2}+\sum_{j=2}^{\ell_{3}} \alpha_{n, j}^{2}\left(\rho^{j}\right)^{2} \prod_{i=1}^{j-1}\left(1-\alpha_{n, i}\right)+\prod_{i=1}^{\ell_{3}}\left(1-\alpha_{n, i}^{2}\right)\left(\rho^{j}\right)^{2}\right) \\
& \times\left(\alpha_{n, 1}^{3}+\sum_{j=2}^{\ell_{4}} \alpha_{n, j}^{3}\left(\rho^{j}\right)^{2} \prod_{i=1}^{j-1}\left(1-\alpha_{n, i}^{3}\right)+\prod_{i=1}^{\ell_{4}}\left(1-\alpha_{n, i}^{3}\right)\left(\rho^{j}\right)^{2}\right)\left[\alpha_{n, 1}^{4}\left\|y_{n}^{5}-q\right\|^{2}\right. \\
& \left.+\sum_{j=2}^{\ell_{5}} \alpha_{n, j}^{4}\left(\rho^{j}\right)^{2} \prod_{i=1}^{j-1}\left(1-\alpha_{n, i}^{4}\right)\left\|y_{n}^{5}-q\right\|^{2}+\prod_{i=1}^{\ell_{5}}\left(1-\alpha_{n, i}^{4}\right)\left(\rho^{j}\right)^{2}\left\|y_{n}^{5}-q\right\|^{2}\right] \\
& =\left(\alpha_{n, 1}^{1}+\sum_{j=2}^{\ell_{2}} \alpha_{n, j}^{1}\left(\rho^{j}\right)^{2} \prod_{i=1}^{j-1}\left(1-\alpha_{n, i}^{1}\right)+\prod_{i=1}^{\ell_{2}}\left(1-\alpha_{n, i}^{1}\right)\left(\rho^{j}\right)^{2}\right) \\
& \times\left(\alpha_{n, 1}^{2}+\sum_{j=2}^{\ell_{3}} \alpha_{n, j}^{2}\left(\rho^{j}\right)^{2} \prod_{i=1}^{j-1}\left(1-\alpha_{n, i}\right)+\prod_{i=1}^{\ell_{3}}\left(1-\alpha_{n, i}^{2}\right)\left(\rho^{j}\right)^{2}\right) \\
& \times\left(\alpha_{n, 1}^{3}+\sum_{j=2}^{\ell_{4}} \alpha_{n, j}^{3}\left(\rho^{j}\right)^{2} \prod_{i=1}^{j-1}\left(1-\alpha_{n, i}^{3}\right)+\prod_{i=1}^{\ell_{4}}\left(1-\alpha_{n, i}^{3}\right)\left(\rho^{j}\right)^{2}\right)
\end{aligned}
$$




$$
\begin{aligned}
& \times\left(\alpha_{n, 1}^{4}+\sum_{j=2}^{\ell_{5}} \alpha_{n, j}^{4}\left(\rho^{j}\right)^{2} \prod_{i=1}^{j-1}\left(1-\alpha_{n, i}^{4}\right)+\prod_{i=1}^{\ell_{5}}\left(1-\alpha_{n, i}^{4}\right)\left(\rho^{j}\right)^{2}\right)\left\|y_{n}^{5}-q\right\|^{2} \\
\leq & \left(\alpha_{n, 1}^{1}+\sum_{j=2}^{\ell_{2}} \alpha_{n, j}^{1}\left(\rho^{j}\right)^{2} \prod_{i=1}^{j-1}\left(1-\alpha_{n, i}^{1}\right)+\prod_{i=1}^{\ell_{2}}\left(1-\alpha_{n, i}^{1}\right)\left(\rho^{j}\right)^{2}\right) \\
& \times\left(\alpha_{n, 1}^{2}+\sum_{j=2}^{\ell_{3}} \alpha_{n, j}^{2}\left(\rho^{j}\right)^{2} \prod_{i=1}^{j-1}\left(1-\alpha_{n, i}\right)+\prod_{i=1}^{\ell_{3}}\left(1-\alpha_{n, i}^{2}\right)\left(\rho^{j}\right)^{2}\right) \\
& \times\left(\alpha_{n, 1}^{3}+\sum_{j=2}^{\ell_{4}} \alpha_{n, j}^{3}\left(\rho^{j}\right)^{2} \prod_{i=1}^{j-1}\left(1-\alpha_{n, i}^{3}\right)+\prod_{i=1}^{\ell_{4}}\left(1-\alpha_{n, i}^{3}\right)\left(\rho^{j}\right)^{2}\right) \\
& \times\left(\alpha_{n, 1}^{4}+\sum_{j=2}^{\ell_{5}} \alpha_{n, j}^{4}\left(\rho^{j}\right)^{2} \prod_{i=1}^{j-1}\left(1-\alpha_{n, i}^{4}\right)+\prod_{i=1}^{\ell_{5}}\left(1-\alpha_{n, i}^{4}\right)\left(\rho^{j}\right)^{2}\right) \\
& \times \cdots \times\left(\alpha_{n, 1}^{\ell_{s-2}}+\sum_{j=2}^{\ell_{s-1}} \alpha_{n, j}^{\ell_{s-2}}\left(\rho^{j}\right)^{2} \prod_{i=1}^{j-1}\left(1-\alpha_{n, i}^{\ell_{s-2}}\right)+\prod_{i=1}^{\ell_{s-1}}\left(1-\alpha_{n, i}^{\ell_{s-2}}\right)\left(\rho^{j}\right)^{2}\right) \\
& \times\left(\alpha_{n, 1}^{\ell_{s-1}}+\sum_{j=2}^{\ell_{s}} \alpha_{n, j}^{\ell_{s-1}}\left(\rho^{j}\right)^{2} \prod_{i=1}^{j-1}\left(1-\alpha_{n, i}^{\ell_{s-1}}\right)+\prod_{i=1}^{\ell_{s}}\left(1-\alpha_{n, i}^{\ell_{s-1}}\right)\left(\rho^{j}\right)^{2}\right) \\
& \times\left\|x_{n}-q\right\|^{2}
\end{aligned}
$$

(3.13) and (3.15) imply that

$$
\begin{aligned}
\left\|x_{n+1}-q\right\|^{2} \leq & \left(\alpha_{n, 1}^{1}+\sum_{j=2}^{\ell_{2}} \alpha_{n, j}^{1}\left(\rho^{j}\right)^{2} \prod_{i=1}^{j-1}\left(1-\alpha_{n, i}^{1}\right)+\prod_{i=1}^{\ell_{2}}\left(1-\alpha_{n, i}^{1}\right)\left(\rho^{j}\right)^{2}\right) \\
& \times\left(\alpha_{n, 1}^{2}+\sum_{j=2}^{\ell_{3}} \alpha_{n, j}^{2}\left(\rho^{j}\right)^{2} \prod_{i=1}^{j-1}\left(1-\alpha_{n, i}\right)+\prod_{i=1}^{\ell_{3}}\left(1-\alpha_{n, i}^{2}\right)\left(\rho^{j}\right)^{2}\right) \\
& \times\left(\alpha_{n, 1}^{3}+\sum_{j=2}^{\ell_{4}} \alpha_{n, j}^{3}\left(\rho^{j}\right)^{2} \prod_{i=1}^{j-1}\left(1-\alpha_{n, i}^{3}\right)+\prod_{i=1}^{\ell_{4}}\left(1-\alpha_{n, i}^{3}\right)\left(\rho^{j}\right)^{2}\right) \\
& \times\left(\alpha_{n, 1}^{4}+\sum_{j=2}^{\ell_{5}} \alpha_{n, j}^{4}\left(\rho^{j}\right)^{2} \prod_{i=1}^{j-1}\left(1-\alpha_{n, i}^{4}\right)+\prod_{i=1}^{\ell_{5}}\left(1-\alpha_{n, i}^{4}\right)\left(\rho^{j}\right)^{2}\right) \\
& \times \cdots \times\left(\alpha_{n, 1}^{\ell_{s-2}}+\sum_{j=2}^{\ell_{s-1}} \alpha_{n, j}^{\ell_{s-2}}\left(\rho^{j}\right)^{2} \prod_{i=1}^{j-1}\left(1-\alpha_{n, i}^{\ell_{s-2}}\right)+\prod_{i=1}^{\ell_{s-1}}\left(1-\alpha_{n, i}^{\ell_{s-2}}\right)\left(\rho^{j}\right)^{2}\right) \\
& \times\left(\alpha_{n, 1}^{\ell_{s-1}}+\sum_{j=2}^{\ell_{s}} \alpha_{n, j}^{\ell_{s-1}}\left(\rho^{j}\right)^{2} \prod_{i=1}^{j-1}\left(1-\alpha_{n, i}^{\ell_{s-1}}\right)+\prod_{i=1}^{\ell_{s}}\left(1-\alpha_{n, i}^{\ell_{s-1}}\right)\left(\rho^{j}\right)^{2}\right) \\
& \times\left\|x_{n}-q\right\|^{2}
\end{aligned}
$$


Since $\rho^{j} \in[0,1]$, we obtain using Proposition 2.3, for $j=1,2,3, \cdots, s-1$, that

$$
Q \leq P=1
$$

where

$$
\begin{aligned}
Q= & \left(\alpha_{n, 1}^{3}+\sum_{j=2}^{\ell_{4}} \alpha_{n, j}^{3}\left(\rho^{j}\right)^{2} \prod_{i=1}^{j-1}\left(1-\alpha_{n, i}^{3}\right)+\prod_{i=1}^{\ell_{4}}\left(1-\alpha_{n, i}^{3}\right)\left(\rho^{j}\right)^{2}\right) \\
& \times\left(\alpha_{n, 1}^{4}+\sum_{j=2}^{\ell_{5}} \alpha_{n, j}^{4}\left(\rho^{j}\right)^{2} \prod_{i=1}^{j-1}\left(1-\alpha_{n, i}^{4}\right)+\prod_{i=1}^{\ell_{5}}\left(1-\alpha_{n, i}^{4}\right)\left(\rho^{j}\right)^{2}\right) \\
& \times \cdots \times\left(\alpha_{n, 1}^{\ell_{s-2}}+\sum_{j=2}^{\ell_{s-1}} \alpha_{n, j}^{\ell_{s-2}}\left(\rho^{j}\right)^{2} \prod_{i=1}^{j-1}\left(1-\alpha_{n, i}^{\ell_{s-2}}\right)+\prod_{i=1}^{\ell_{s-1}}\left(1-\alpha_{n, i}^{\ell_{s-2}}\right)\left(\rho^{j}\right)^{2}\right) \\
& \times\left(\alpha_{n, 1}^{\ell_{s-1}}+\sum_{j=2}^{\ell_{s}} \alpha_{n, j}^{\ell_{s-1}}\left(\rho^{j}\right)^{2} \prod_{i=1}^{j-1}\left(1-\alpha_{n, i}^{\ell_{s-1}}\right)+\prod_{i=1}^{\ell_{s}}\left(1-\alpha_{n, i}^{\ell_{s-1}}\right)\left(\rho^{j}\right)^{2}\right)
\end{aligned}
$$

and

$$
\begin{aligned}
P= & \left(\alpha_{n, 1}^{3}+\sum_{j=2}^{\ell_{4}} \alpha_{n, j}^{3} \prod_{i=1}^{j-1}\left(1-\alpha_{n, i}^{3}\right)+\prod_{i=1}^{\ell_{4}}\left(1-\alpha_{n, i}^{3}\right)\right) \\
& \times\left(\alpha_{n, 1}^{4}+\sum_{j=2}^{\ell_{5}} \alpha_{n, j}^{4} \prod_{i=1}^{j-1}\left(1-\alpha_{n, i}^{4}\right)+\prod_{i=1}^{\ell_{5}}\left(1-\alpha_{n, i}^{4}\right)\right) \\
& \times \cdots \times\left(\alpha_{n, 1}^{\ell_{s-2}}+\sum_{j=2}^{\ell_{s-1}} \alpha_{n, j}^{\ell_{s-2}} \prod_{i=1}^{j-1}\left(1-\alpha_{n, i}^{\ell_{s-2}}\right)+\prod_{i=1}^{\ell_{s-1}}\left(1-\alpha_{n, i}^{\ell_{s-2}}\right)\right) \\
& \times\left(\alpha_{n, 1}^{\ell_{s-1}}+\sum_{j=2}^{\ell_{s}} \alpha_{n, j}^{\ell_{s-1}} \prod_{i=1}^{j-1}\left(1-\alpha_{n, i}^{\ell_{s-1}}\right)+\prod_{i=1}^{\ell_{s}}\left(1-\alpha_{n, i}^{\ell_{s-1}}\right)\right)
\end{aligned}
$$

Applying (3.17) in (3.16), we obtain, using Lemma 2.3 that the sequence $\left\{x_{n}\right\}_{n=0}^{\infty}$ defined by (3.2) converges strongly to the fixed point $q$ in $F(\Gamma)$. Thus, the proof is completed.

Example 3.1. Let the operator $\Gamma:[0,1] \longrightarrow[0,1]$ be defined as

$$
\left\ulcorner z=\frac{z}{3}, \forall z \in[0,1] .\right.
$$

Clearly, $\Gamma$ is quasi-contractive satisfying (2.2) with a unique fixed point 0; see, for example, [26] for details. Set

$$
\begin{array}{r}
\alpha_{n, 1}^{1}=\delta_{n, 1}^{1}=\frac{1}{\sqrt{n+1}}, n=1,2, \cdots, n_{0}, \text { for } n_{0} \in \mathbb{N} ; \\
\delta_{n, i}=1-\delta_{n, 1}^{1}, \text { for } i=1,2, \cdots, \ell_{1} \text { and } \\
\alpha_{n, i}^{s}=1-2 \alpha_{n, 1}^{1}, \text { for } i=1,2, \cdots, \ell_{s+1}, s=1,2, \cdots, n_{0} .
\end{array}
$$

It is not hard to see that all the conditions of Theorem 3.1 and Theorem 3.2 has been satisfied by Example 3.1. 


\section{Main Results II}

Here, we consider stablity results for the multistep $/ H$-iteration scheme and the multistep $D /$ iteration scheme defined by (3.1) and (3.2) for operators satisfying (2.2), respectively.

Theorem 4.1. Let $H$ be a Hilbert space, $\Gamma: H \longrightarrow H$ be a self-map of $H$ satisfying the contractive condition

$$
\left\|\Gamma^{j} x-\Gamma^{j} y\right\| \leq \rho^{j}\|x-y\|+\sum_{i=0}^{j}\left(\begin{array}{c}
j \\
i
\end{array}\right) \rho^{j-1} \phi(\|x-\Gamma x\|),
$$

where $x, y \in H, 0 \leq \rho^{j}<1$, and let $\phi$ retains its usual meaning with $\phi(0)=0$ and $\phi(M t)=$ $M \phi(t), M \geq 0, t \in \mathbb{R}^{+}$. For arbitrary $x_{0} \in H$, let $\left\{x_{n}\right\}_{n=0}^{\infty}$ be the multistep DI-iteration scheme defined by (3.2). Assume $F(\Gamma) \neq \emptyset, q \in F(\Gamma)$. Then, the multisetp DI-iterative scheme is $\Gamma$-stable.

Proof. Let $\left\{v_{n}\right\}_{n=0}^{\infty}$, be a real sequences in $H$. Suppose $\left\{t_{n}\right\}_{n=0}^{\infty} \subset X$ is an arbitrary sequence, set

$$
\epsilon_{n}=\left\|t_{n+1}-\delta_{n, 1} v_{n, 1}^{1}-\sum_{j=2}^{\ell_{1}} \delta_{n, j} \prod_{i=1}^{j-1}\left(1-\delta_{n, i}\right) \Gamma^{j-1} v_{n}^{1}-\prod_{i=1}^{\ell_{1}}\left(1-\delta_{n, i}\right) \Gamma^{\ell_{1}} v_{n}^{1}\right\|^{2}
$$

where, for $s=1,2, \cdots, k-2$,

$$
v_{n}^{s}=\alpha_{n, 1}^{s} v_{n}^{s+1}+\sum_{j=2}^{\ell_{s+1}} \alpha_{n, j}^{s} \prod_{i=1}^{j-1}\left(1-\alpha_{n, i}^{s}\right) \Gamma^{j-1} v_{n}^{s+1}+\prod_{i=1}^{\ell_{s+1}}\left(1-\alpha_{n, i}^{s}\right) \Gamma^{\ell_{1}} v_{n}^{s+1}
$$

and, for $k \geq 2$,

$$
v_{n}^{k-1}=\sum_{j=1}^{\ell_{k}} \alpha_{n, j}^{k-1} \prod_{i=1}^{j-1}\left(1-\alpha_{n, i}^{k-1}\right) \Gamma^{j-1} t_{n}+\prod_{i=1}^{\ell_{k}}\left(1-\alpha_{n, i}^{k-1}\right) \Gamma^{\ell_{k}} t_{n}, n \geq 1,
$$

Now, suppose $\epsilon_{n} \rightarrow 0$ as $n \rightarrow \infty$. Then, we show that $t_{n} \rightarrow q$ as $n \rightarrow \infty$ using contractive mapping defined by (4.1).

Indeed, using Proposition 2.4 with $u=q, v_{n}^{1}=t, j=i, k=1, \Gamma^{j-1} v_{n}^{1}=v_{j-1}$ and $\Gamma^{\ell_{1}} v_{n}^{1}=v_{n}$ we obtain

$$
\begin{aligned}
\left\|t_{n+1}-q\right\|^{2}= & \| \delta_{n, 1} v_{n, 1}^{1}+\sum_{j=2}^{\ell_{1}} \delta_{n, j} \prod_{i=1}^{j-1}\left(1-\delta_{n, i}\right) \Gamma^{j-1} v_{n}^{1}+\prod_{i=1}^{\ell_{1}}\left(1-\delta_{n, i}\right) \Gamma^{\ell_{1}} v_{n}^{1}-q \\
& -\left[\delta_{n, 1} v_{n, 1}^{1}+\sum_{j=2}^{\ell_{1}} \delta_{n, j} \prod_{i=1}^{j-1}\left(1-\delta_{n, i}\right) \Gamma^{j-1} v_{n}^{1}+\prod_{i=1}^{\ell_{1}}\left(1-\delta_{n, i}\right) \Gamma^{\ell_{1}} v_{n}^{1}-t_{n+1}\right] \|^{2}
\end{aligned}
$$




$$
\begin{aligned}
& \leq\left\|-\left[t_{n+1}-\delta_{n, 1} v_{n, 1}^{1}-\sum_{j=2}^{\ell_{1}} \delta_{n, j} \prod_{i=1}^{j-1}\left(1-\delta_{n, i}\right) \Gamma^{j-1} v_{n}^{1}-\prod_{i=1}^{\ell_{1}}\left(1-\delta_{n, i}\right) \Gamma^{\ell_{1}} v_{n}^{1}\right]\right\|^{2} \\
& +\left\|\delta_{n, 1} v_{n, 1}^{1}+\sum_{j=2}^{\ell_{1}} \delta_{n, j} \prod_{i=1}^{j-1}\left(1-\delta_{n, i}\right) \Gamma^{j-1} v_{n}^{1}+\prod_{i=1}^{\ell_{1}}\left(1-\delta_{n, i}\right) \Gamma^{\ell_{1}} v_{n}^{1}-q\right\|^{2} \\
& =\left\|t_{n+1}-\delta_{n, 1} v_{n, 1}^{1}-\sum_{j=2}^{\ell_{1}} \delta_{n, j} \prod_{i=1}^{j-1}\left(1-\delta_{n, i}\right) \Gamma^{j-1} v_{n}^{1}-\prod_{i=1}^{\ell_{1}}\left(1-\delta_{n, i}\right) \Gamma^{\ell_{1}} v_{n}^{1}\right\|^{2} \\
& +\left\|\delta_{n, 1} v_{n, 1}^{1}+\sum_{j=2}^{\ell_{1}} \delta_{n, j} \prod_{i=1}^{j-1}\left(1-\delta_{n, i}\right) \Gamma^{j-1} v_{n}^{1}+\prod_{i=1}^{\ell_{1}}\left(1-\delta_{n, i}\right) \Gamma^{\ell_{1}} v_{n}^{1}-q\right\|^{2} \\
& =\epsilon_{n}+\left\|\delta_{n, 1} v_{n, 1}^{1}+\sum_{j=2}^{\ell_{1}} \delta_{n, j} \prod_{i=1}^{j-1}\left(1-\delta_{n, i}\right) \Gamma^{j-1} v_{n}^{1}+\prod_{i=1}^{\ell_{1}}\left(1-\delta_{n, i}\right) \Gamma^{\ell_{1}} v_{n}^{1}-q\right\|^{2} \\
& \leq \epsilon_{n}+\delta_{n, 1}\left\|v_{n, 1}^{1}-q\right\|^{2}+\sum_{j=2}^{\ell_{1}} \delta_{n, j} \prod_{i=1}^{j-1}\left(1-\delta_{n, i}\right)\left\|\Gamma^{j-1} v_{n}^{1}-q\right\|^{2} \\
& +\prod_{i=1}^{\ell_{1}}\left(1-\delta_{n, i}\right)\left\|\Gamma^{\ell_{1}} v_{n}^{1}-q\right\|^{2} \\
& \leq \epsilon_{n}+\delta_{n, 1}\left\|v_{n, 1}^{1}-q\right\|^{2}+\sum_{j=2}^{\ell_{1}} \delta_{n, j}\left(\rho^{j}\right)^{2} \prod_{i=1}^{j-1}\left(1-\delta_{n, i}\right)\left\|v_{n}^{1}-q\right\|^{2} \\
& +\prod_{i=1}^{\ell_{1}}\left(1-\delta_{n, i}\right)\left(\rho^{j}\right)^{2}\left\|v_{n}^{1}-q\right\|^{2} \\
& \leq \epsilon_{n}+\left(\delta_{n, 1}+\sum_{j=2}^{\ell_{1}} \delta_{n, j}\left(\rho^{j}\right)^{2} \prod_{i=1}^{j-1}\left(1-\delta_{n, i}\right)+\prod_{i=1}^{\ell_{1}}\left(1-\delta_{n, i}\right)\left(\rho^{j}\right)^{2}\right) \\
& \times\left\|v_{n}^{1}-q\right\|^{2}
\end{aligned}
$$

Since $\ell_{1}, \ell_{k}$ are fixed integers and $\alpha_{n, i}^{s} \in[0,1]$ for each $s$, using (3.2) and (3.12), the estimations below are obtained, for $n=1,2, \cdots$ and $1 \leq s \leq k-1$ :,

$$
\begin{aligned}
\left\|v_{n}^{1}-q\right\|^{2} \leq & \alpha_{n, 1}^{1}\left\|v_{n}^{2}-q\right\|^{2}+\sum_{j=2}^{\ell_{2}} \alpha_{n, j}^{1} \prod_{i=1}^{j-1}\left(1-\alpha_{n, i}^{1}\right)\left\|\Gamma^{j-1} v_{n}^{2}-\Gamma^{j-1} q\right\|^{2} \\
& +\prod_{i=1}^{\ell_{2}}\left(1-\alpha_{n, i}^{1}\right)\left\|\Gamma^{\ell_{2}} v_{n}^{2}-\Gamma^{\ell_{2}} q\right\|^{2} \\
\leq & \left(\alpha_{n, 1}^{1}+\sum_{j=2}^{\ell_{2}} \alpha_{n, j}^{1}\left(\rho^{j}\right)^{2} \prod_{i=1}^{j-1}\left(1-\alpha_{n, i}^{1}\right)+\prod_{i=1}^{\ell_{2}}\left(1-\alpha_{n, i}^{1}\right)\left(\rho^{j}\right)^{2}\right)\left\|v_{n}^{2}-q\right\|^{2}
\end{aligned}
$$




$$
\begin{aligned}
& \leq\left(\alpha_{n, 1}^{1}+\sum_{j=2}^{\ell_{2}} \alpha_{n, j}^{2}\left(\rho^{j}\right)^{2} \prod_{i=1}^{j-1}\left(1-\alpha_{n, i}^{1}\right)+\prod_{i=1}^{\ell_{2}}\left(1-\alpha_{n, i}^{1}\right)\left(\rho^{j}\right)^{2}\right)\left[\alpha_{n, 1}^{2}\left\|v_{n}^{3}-q\right\|^{2}\right. \\
& \left.+\sum_{j=2}^{\ell_{3}} \alpha_{n, j}^{2} \prod_{i=1}^{j-1}\left(1-\alpha_{n, i}^{2}\right)\left\|\Gamma^{j-1} v_{n}^{3}-\Gamma^{j-1} q\right\|^{2}+\prod_{i=1}^{\ell_{3}}\left(1-\alpha_{n, i}^{2}\right)\left\|\Gamma^{\ell_{3}} v_{n}^{3}-\Gamma^{\ell_{3}} q\right\|^{2}\right] \\
& \leq\left(\alpha_{n, 1}^{1}+\sum_{j=2}^{\ell_{2}} \alpha_{n, j}^{1}\left(\rho^{j}\right)^{2} \prod_{i=1}^{j-1}\left(1-\alpha_{n, i}^{1}\right)+\prod_{i=1}^{\ell_{2}}\left(1-\alpha_{n, i}^{1}\right)\left(\rho^{j}\right)^{2}\right)\left[\alpha_{n, 1}^{2}\left\|v_{n}^{3}-q\right\|^{2}\right. \\
& \left.+\sum_{j=2}^{\ell_{3}} \alpha_{n, j}^{2}\left(\rho^{j}\right)^{2} \prod_{i=1}^{j-1}\left(1-\alpha_{n, i}\right)\left\|v_{n}^{3}-q\right\|^{2}+\prod_{i=1}^{\ell_{3}}\left(1-\alpha_{n, i}^{2}\right)\left(\rho^{j}\right)^{2}\left\|v_{n}^{3}-q\right\|^{2}\right] \\
& =\left(\alpha_{n, 1}^{1}+\sum_{j=2}^{\ell_{2}} \alpha_{n, j}^{1}\left(\rho^{j}\right)^{2} \prod_{i=1}^{j-1}\left(1-\alpha_{n, i}^{1}\right)+\prod_{i=1}^{\ell_{2}}\left(1-\alpha_{n, i}^{1}\right)\left(\rho^{j}\right)^{2}\right) \\
& \times\left(\alpha_{n, 1}^{2}+\sum_{j=2}^{\ell_{3}} \alpha_{n, j}^{2}\left(\rho^{j}\right)^{2} \prod_{i=1}^{j-1}\left(1-\alpha_{n, i}\right)+\prod_{i=1}^{\ell_{3}}\left(1-\alpha_{n, i}^{2}\right)\left(\rho^{j}\right)^{2}\right)\left\|v_{n}^{3}-q\right\|^{2} \\
& \leq\left(\alpha_{n, 1}^{1}+\sum_{j=2}^{\ell_{2}} \alpha_{n, j}^{1}\left(\rho^{j}\right)^{2} \prod_{i=1}^{j-1}\left(1-\alpha_{n, i}^{1}\right)+\prod_{i=1}^{\ell_{2}}\left(1-\alpha_{n, i}^{1}\right)\left(\rho^{j}\right)^{2}\right) \\
& \times\left(\alpha_{n, 1}^{2}+\sum_{j=2}^{\ell_{3}} \alpha_{n, j}^{2}\left(\rho^{j}\right)^{2} \prod_{i=1}^{j-1}\left(1-\alpha_{n, i}\right)+\prod_{i=1}^{\ell_{3}}\left(1-\alpha_{n, i}^{2}\right)\left(\rho^{j}\right)^{2}\right)\left[\alpha_{n, 1}^{3}\left\|v_{n}^{4}-q\right\|^{2}\right. \\
& \left.+\sum_{j=2}^{\ell_{4}} \alpha_{n, j}^{3} \prod_{i=1}^{j-1}\left(1-\alpha_{n, i}^{3}\right)\left\|\Gamma^{j-1} v_{n}^{4}-\Gamma^{j-1} q\right\|^{2}+\prod_{i=1}^{\ell_{4}}\left(1-\alpha_{n, i}^{3}\right)\left\|\Gamma^{\ell_{4}} v_{n}^{4}-\Gamma^{\ell_{4}} q\right\|^{2}\right] \\
& \leq\left(\alpha_{n, 1}^{1}+\sum_{j=2}^{\ell_{2}} \alpha_{n, j}^{1}\left(\rho^{j}\right)^{2} \prod_{i=1}^{j-1}\left(1-\alpha_{n, i}^{1}\right)+\prod_{i=1}^{\ell_{2}}\left(1-\alpha_{n, i}^{1}\right)\left(\rho^{j}\right)^{2}\right) \\
& \times\left(\alpha_{n, 1}^{2}+\sum_{j=2}^{\ell_{3}} \alpha_{n, j}^{2}\left(\rho^{j}\right)^{2} \prod_{i=1}^{j-1}\left(1-\alpha_{n, i}\right)+\prod_{i=1}^{\ell_{3}}\left(1-\alpha_{n, i}^{2}\right)\left(\rho^{j}\right)^{2}\right)\left[\alpha_{n, 1}^{3}\left\|v_{n}^{4}-q\right\|^{2}\right. \\
& \left.+\sum_{j=2}^{\ell_{4}} \alpha_{n, j}^{3}\left(\rho^{j}\right)^{2} \prod_{i=1}^{j-1}\left(1-\alpha_{n, i}^{3}\right)\left\|v_{n}^{4}-q\right\|^{2}+\prod_{i=1}^{\ell_{4}}\left(1-\alpha_{n, i}^{3}\right)\left(\rho^{j}\right)^{2}\left\|v_{n}^{4}-q\right\|^{2}\right] \\
& =\left(\alpha_{n, 1}^{1}+\sum_{j=2}^{\ell_{2}} \alpha_{n, j}^{1}\left(\rho^{j}\right)^{2} \prod_{i=1}^{j-1}\left(1-\alpha_{n, i}^{1}\right)+\prod_{i=1}^{\ell_{2}}\left(1-\alpha_{n, i}^{1}\right)\left(\rho^{j}\right)^{2}\right) \\
& \times\left(\alpha_{n, 1}^{2}+\sum_{j=2}^{\ell_{3}} \alpha_{n, j}^{2}\left(\rho^{j}\right)^{2} \prod_{i=1}^{j-1}\left(1-\alpha_{n, i}\right)+\prod_{i=1}^{\ell_{3}}\left(1-\alpha_{n, i}^{2}\right)\left(\rho^{j}\right)^{2}\right) \\
& \times\left(\alpha_{n, 1}^{3}+\sum_{j=2}^{\ell_{4}} \alpha_{n, j}^{3}\left(\rho^{j}\right)^{2} \prod_{i=1}^{j-1}\left(1-\alpha_{n, i}^{3}\right)+\prod_{i=1}^{\ell_{4}}\left(1-\alpha_{n, i}^{3}\right)\left(\rho^{j}\right)^{2}\right)\left\|v_{n}^{4}-q\right\|^{2}
\end{aligned}
$$




$$
\begin{aligned}
& \leq\left(\alpha_{n, 1}^{1}+\sum_{j=2}^{\ell_{2}} \alpha_{n, j}^{1}\left(\rho^{j}\right)^{2} \prod_{i=1}^{j-1}\left(1-\alpha_{n, i}^{1}\right)+\prod_{i=1}^{\ell_{2}}\left(1-\alpha_{n, i}^{1}\right)\left(\rho^{j}\right)^{2}\right) \\
& \times\left(\alpha_{n, 1}^{2}+\sum_{j=2}^{\ell_{3}} \alpha_{n, j}^{2}\left(\rho^{j}\right)^{2} \prod_{i=1}^{j-1}\left(1-\alpha_{n, i}\right)+\prod_{i=1}^{\ell_{3}}\left(1-\alpha_{n, i}^{2}\right)\left(\rho^{j}\right)^{2}\right) \\
& \times\left(\alpha_{n, 1}^{3}+\sum_{j=2}^{\ell_{4}} \alpha_{n, j}^{3}\left(\rho^{j}\right)^{2} \prod_{i=1}^{j-1}\left(1-\alpha_{n, i}^{3}\right)+\prod_{i=1}^{\ell_{4}}\left(1-\alpha_{n, i}^{3}\right)\left(\rho^{j}\right)^{2}\right)\left[\alpha_{n, 1}^{4}\left\|v_{n}^{5}-q\right\|^{2}\right. \\
& \left.+\sum_{j=2}^{\ell_{5}} \alpha_{n, j}^{4} \prod_{i=1}^{j-1}\left(1-\alpha_{n, i}^{4}\right)\left\|\Gamma^{j-1} v_{n}^{5}-\Gamma^{j-1} q\right\|^{2}+\prod_{i=1}^{\ell_{5}}\left(1-\alpha_{n, i}^{4}\right)\left\|\Gamma^{\ell_{5}} v_{n}^{5}-\Gamma^{\ell_{5}} q\right\|^{2}\right] \\
& \leq\left(\alpha_{n, 1}^{1}+\sum_{j=2}^{\ell_{2}} \alpha_{n, j}^{1}\left(\rho^{j}\right)^{2} \prod_{i=1}^{j-1}\left(1-\alpha_{n, i}^{1}\right)+\prod_{i=1}^{\ell_{2}}\left(1-\alpha_{n, i}^{1}\right)\left(\rho^{j}\right)^{2}\right) \\
& \times\left(\alpha_{n, 1}^{2}+\sum_{j=2}^{\ell_{3}} \alpha_{n, j}^{2}\left(\rho^{j}\right)^{2} \prod_{i=1}^{j-1}\left(1-\alpha_{n, i}\right)+\prod_{i=1}^{\ell_{3}}\left(1-\alpha_{n, i}^{2}\right)\left(\rho^{j}\right)^{2}\right) \\
& \times\left(\alpha_{n, 1}^{3}+\sum_{j=2}^{\ell_{4}} \alpha_{n, j}^{3}\left(\rho^{j}\right)^{2} \prod_{i=1}^{j-1}\left(1-\alpha_{n, i}^{3}\right)+\prod_{i=1}^{\ell_{4}}\left(1-\alpha_{n, i}^{3}\right)\left(\rho^{j}\right)^{2}\right)\left[\alpha_{n, 1}^{4}\left\|v_{n}^{5}-q\right\|^{2}\right. \\
& \left.+\sum_{j=2}^{\ell_{5}} \alpha_{n, j}^{4}\left(\rho^{j}\right)^{2} \prod_{i=1}^{j-1}\left(1-\alpha_{n, i}^{4}\right)\left\|v_{n}^{5}-q\right\|^{2}+\prod_{i=1}^{\ell_{5}}\left(1-\alpha_{n, i}^{4}\right)\left(\rho^{j}\right)^{2}\left\|v_{n}^{5}-q\right\|^{2}\right] \\
& =\left(\alpha_{n, 1}^{1}+\sum_{j=2}^{\ell_{2}} \alpha_{n, j}^{1}\left(\rho^{j}\right)^{2} \prod_{i=1}^{j-1}\left(1-\alpha_{n, i}^{1}\right)+\prod_{i=1}^{\ell_{2}}\left(1-\alpha_{n, i}^{1}\right)\left(\rho^{j}\right)^{2}\right) \\
& \times\left(\alpha_{n, 1}^{2}+\sum_{j=2}^{\ell_{3}} \alpha_{n, j}^{2}\left(\rho^{j}\right)^{2} \prod_{i=1}^{j-1}\left(1-\alpha_{n, i}\right)+\prod_{i=1}^{\ell_{3}}\left(1-\alpha_{n, i}^{2}\right)\left(\rho^{j}\right)^{2}\right) \\
& \times\left(\alpha_{n, 1}^{3}+\sum_{j=2}^{\ell_{4}} \alpha_{n, j}^{3}\left(\rho^{j}\right)^{2} \prod_{i=1}^{j-1}\left(1-\alpha_{n, i}^{3}\right)+\prod_{i=1}^{\ell_{4}}\left(1-\alpha_{n, i}^{3}\right)\left(\rho^{j}\right)^{2}\right) \\
& \times\left(\alpha_{n, 1}^{4}+\sum_{j=2}^{\ell_{5}} \alpha_{n, j}^{4}\left(\rho^{j}\right)^{2} \prod_{i=1}^{j-1}\left(1-\alpha_{n, i}^{4}\right)+\prod_{i=1}^{\ell_{5}}\left(1-\alpha_{n, i}^{4}\right)\left(\rho^{j}\right)^{2}\right)\left\|v_{n}^{5}-q\right\|^{2} \\
& \leq\left(\alpha_{n, 1}^{1}+\sum_{j=2}^{\ell_{2}} \alpha_{n, j}^{1}\left(\rho^{j}\right)^{2} \prod_{i=1}^{j-1}\left(1-\alpha_{n, i}^{1}\right)+\prod_{i=1}^{\ell_{2}}\left(1-\alpha_{n, i}^{1}\right)\left(\rho^{j}\right)^{2}\right) \\
& \times\left(\alpha_{n, 1}^{2}+\sum_{j=2}^{\ell_{3}} \alpha_{n, j}^{2}\left(\rho^{j}\right)^{2} \prod_{i=1}^{j-1}\left(1-\alpha_{n, i}\right)+\prod_{i=1}^{\ell_{3}}\left(1-\alpha_{n, i}^{2}\right)\left(\rho^{j}\right)^{2}\right) \\
& \times\left(\alpha_{n, 1}^{3}+\sum_{j=2}^{\ell_{4}} \alpha_{n, j}^{3}\left(\rho^{j}\right)^{2} \prod_{i=1}^{j-1}\left(1-\alpha_{n, i}^{3}\right)+\prod_{i=1}^{\ell_{4}}\left(1-\alpha_{n, i}^{3}\right)\left(\rho^{j}\right)^{2}\right) \\
& \times\left(\alpha_{n, 1}^{4}+\sum_{j=2}^{\ell_{5}} \alpha_{n, j}^{4}\left(\rho^{j}\right)^{2} \prod_{i=1}^{j-1}\left(1-\alpha_{n, i}^{4}\right)+\prod_{i=1}^{\ell_{5}}\left(1-\alpha_{n, i}^{4}\right)\left(\rho^{j}\right)^{2}\right)
\end{aligned}
$$




$$
\begin{aligned}
& \times \cdots \times\left(\alpha_{n, 1}^{\ell_{s-2}}+\sum_{j=2}^{\ell_{s-1}} \alpha_{n, j}^{\ell_{s-2}}\left(\rho^{j}\right)^{2} \prod_{i=1}^{j-1}\left(1-\alpha_{n, i}^{\ell_{s-2}}\right)+\prod_{i=1}^{\ell_{s-1}}\left(1-\alpha_{n, i}^{\ell_{s-2}}\right)\left(\rho^{j}\right)^{2}\right) \\
& \times\left(\alpha_{n, 1}^{\ell_{s-1}}+\sum_{j=2}^{\ell_{s}} \alpha_{n, j}^{\ell_{s-1}}\left(\rho^{j}\right)^{2} \prod_{i=1}^{j-1}\left(1-\alpha_{n, i}^{\ell_{s-1}}\right)+\prod_{i=1}^{\ell_{s}}\left(1-\alpha_{n, i}^{\ell_{s-1}}\right)\left(\rho^{j}\right)^{2}\right) \\
& \times\left\|t_{n}-q\right\|^{2}
\end{aligned}
$$

(4.5) and (4.6)imply that

$$
\begin{aligned}
\left\|t_{n+1}-q\right\|^{2} \leq & \left(\delta_{n, 1}+\sum_{j=2}^{\ell_{1}} \delta_{n, j}\left(\rho^{j}\right)^{2} \prod_{i=1}^{j-1}\left(1-\delta_{n, i}\right)+\prod_{i=1}^{\ell_{1}}\left(1-\delta_{n, i}\right)\left(\rho^{j}\right)^{2}\right) \\
& \times\left(\alpha_{n, 1}^{1}+\sum_{j=2}^{\ell_{2}} \alpha_{n, j}^{1}\left(\rho^{j}\right)^{2} \prod_{i=1}^{j-1}\left(1-\alpha_{n, i}^{1}\right)+\prod_{i=1}^{\ell_{2}}\left(1-\alpha_{n, i}^{1}\right)\left(\rho^{j}\right)^{2}\right) \\
& \times\left(\alpha_{n, 1}^{2}+\sum_{j=2}^{\ell_{3}} \alpha_{n, j}^{2}\left(\rho^{j}\right)^{2} \prod_{i=1}^{j-1}\left(1-\alpha_{n, i}\right)+\prod_{i=1}^{\ell_{3}}\left(1-\alpha_{n, i}^{2}\right)\left(\rho^{j}\right)^{2}\right) \\
& \times\left(\alpha_{n, 1}^{3}+\sum_{j=2}^{\ell_{4}} \alpha_{n, j}^{3}\left(\rho^{j}\right)^{2} \prod_{i=1}^{j-1}\left(1-\alpha_{n, i}^{3}\right)+\prod_{i=1}^{\ell_{4}}\left(1-\alpha_{n, i}^{3}\right)\left(\rho^{j}\right)^{2}\right) \\
& \times\left(\alpha_{n, 1}^{4}+\sum_{j=2}^{\ell_{5}} \alpha_{n, j}^{4}\left(\rho^{j}\right)^{2} \prod_{i=1}^{j-1}\left(1-\alpha_{n, i}^{4}\right)+\prod_{i=1}^{\ell_{5}}\left(1-\alpha_{n, i}^{4}\right)\left(\rho^{j}\right)^{2}\right) \\
& \times \cdots \times\left(\alpha_{n, 1}^{\ell_{s-2}}+\sum_{j=2}^{\ell_{s-1}} \alpha_{n, j}^{\ell_{s-2}}\left(\rho^{j}\right)^{2} \prod_{i=1}^{j-1}\left(1-\alpha_{n, i}^{\ell_{s-2}}\right)+\prod_{i=1}^{\ell_{s-1}}\left(1-\alpha_{n, i}^{\ell_{s-2}}\right)\left(\rho^{j}\right)^{2}\right) \\
& \times\left(\alpha_{n, 1}^{\ell_{s-1}}+\sum_{j=2}^{\ell_{s}} \alpha_{n, j}^{\ell_{s-1}}\left(\rho^{j}\right)^{2} \prod_{i=1}^{j-1}\left(1-\alpha_{n, i}^{\ell_{s-1}}\right)+\prod_{i=1}^{\ell_{s}}\left(1-\alpha_{n, i}^{\ell_{s-1}}\right)\left(\rho^{j}\right)^{2}\right) \\
& \times\left\|t_{n}-q\right\|^{2}+\epsilon_{n}
\end{aligned}
$$

Note that (4.7) is valid since $\Gamma q=q$ and $\phi(0)=0$.

Now, since $\rho^{j} \in[0,1]$, we obtain using Proposition 2.3, for $j=1,2,3, \cdots, s-1$, that

$$
\tau_{n}<\eta_{n}=1
$$

where

$$
\begin{aligned}
\tau_{n}= & \left(\alpha_{n, 1}^{3}+\sum_{j=2}^{\ell_{4}} \alpha_{n, j}^{3}\left(\rho^{j}\right)^{2} \prod_{i=1}^{j-1}\left(1-\alpha_{n, i}^{3}\right)+\prod_{i=1}^{\ell_{4}}\left(1-\alpha_{n, i}^{3}\right)\left(\rho^{j}\right)^{2}\right) \\
& \times\left(\alpha_{n, 1}^{4}+\sum_{j=2}^{\ell_{5}} \alpha_{n, j}^{4}\left(\rho^{j}\right)^{2} \prod_{i=1}^{j-1}\left(1-\alpha_{n, i}^{4}\right)+\prod_{i=1}^{\ell_{5}}\left(1-\alpha_{n, i}^{4}\right)\left(\rho^{j}\right)^{2}\right) \\
& \times \cdots \times\left(\alpha_{n, 1}^{\ell_{s-2}}+\sum_{j=2}^{\ell_{s-1}} \alpha_{n, j}^{\ell_{s-2}}\left(\rho^{j}\right)^{2} \prod_{i=1}^{j-1}\left(1-\alpha_{n, i}^{\ell_{s-2}}\right)+\prod_{i=1}^{\ell_{s-1}}\left(1-\alpha_{n, i}^{\ell_{s-2}}\right)\left(\rho^{j}\right)^{2}\right)
\end{aligned}
$$




$$
\times\left(\alpha_{n, 1}^{\ell_{s-1}}+\sum_{j=2}^{\ell_{s}} \alpha_{n, j}^{\ell_{s-1}}\left(\rho^{j}\right)^{2} \prod_{i=1}^{j-1}\left(1-\alpha_{n, i}^{\ell_{s-1}}\right)+\prod_{i=1}^{\ell_{s}}\left(1-\alpha_{n, i}^{\ell_{s-1}}\right)\left(\rho^{j}\right)^{2}\right)
$$

and

$$
\begin{aligned}
\eta_{n}= & \left(\alpha_{n, 1}^{3}+\sum_{j=2}^{\ell_{4}} \alpha_{n, j}^{3} \prod_{i=1}^{j-1}\left(1-\alpha_{n, i}^{3}\right)+\prod_{i=1}^{\ell_{4}}\left(1-\alpha_{n, i}^{3}\right)\right) \\
& \times\left(\alpha_{n, 1}^{4}+\sum_{j=2}^{\ell_{5}} \alpha_{n, j}^{4} \prod_{i=1}^{j-1}\left(1-\alpha_{n, i}^{4}\right)+\prod_{i=1}^{\ell_{5}}\left(1-\alpha_{n, i}^{4}\right)\right) \\
& \times \cdots \times\left(\alpha_{n, 1}^{\ell_{s-2}}+\sum_{j=2}^{\ell_{s-1}} \alpha_{n, j}^{\ell_{s-2}} \prod_{i=1}^{j-1}\left(1-\alpha_{n, i}^{\ell_{s-2}}\right)+\prod_{i=1}^{\ell_{s-1}}\left(1-\alpha_{n, i}^{\ell_{s-2}}\right)\right) \\
& \times\left(\alpha_{n, 1}^{\ell_{s-1}}+\sum_{j=2}^{\ell_{s}} \alpha_{n, j}^{\ell_{s-1}} \prod_{i=1}^{j-1}\left(1-\alpha_{n, i}^{\ell_{s-1}}\right)+\prod_{i=1}^{\ell_{s}}\left(1-\alpha_{n, i}^{\ell_{s-1}}\right)\right)
\end{aligned}
$$

Putting (4.8) in (4.7), we obtain, using Lemma 2.3 that the sequence $\left\{t_{n}\right\}_{n=0}^{\infty}$ converges strongly to the point $q$ in $F(\Gamma)$.

On the other hand, suppose $t_{n} \rightarrow q$ as $n \rightarrow \infty$. Then, we show that $\epsilon \rightarrow 0$ as $n \rightarrow \infty$. Indeed, from (3.5) with $v_{n}^{1}=y_{n}^{1},(4.2)$ and Proposition 2.4 with $u=q, v_{n}^{1}=t, j=i, k=1, \Gamma^{j-1} v_{n}^{1}=v_{j-1}$ and $\Gamma^{\ell_{1}} v_{n}^{1}=v_{\text {, we have }}$

$$
\begin{aligned}
\epsilon_{n}= & \left\|t_{n+1}-\delta_{n, 1} v_{n, 1}^{1}-\sum_{j=2}^{\ell_{1}} \delta_{n, j} \prod_{i=1}^{j-1}\left(1-\delta_{n, i}\right) \Gamma^{j-1} v_{n}^{1}-\prod_{i=1}^{\ell_{1}}\left(1-\delta_{n, i}\right) \Gamma^{\ell_{1}} v_{n}^{1}\right\|^{2} \\
= & \left\|t_{n+1}-q-\left(\delta_{n, 1} v_{n, 1}^{1}+\sum_{j=2}^{\ell_{1}} \delta_{n, j} \prod_{i=1}^{j-1}\left(1-\delta_{n, i}\right) \Gamma^{j-1} v_{n}^{1}+\prod_{i=1}^{\ell_{1}}\left(1-\delta_{n, i}\right) \Gamma^{\ell_{1}} v_{n}^{1}-q\right)\right\|^{2} \\
\leq & \left\|t_{n+1}-q\right\|^{2}+\left\|\delta_{n, 1} v_{n, 1}^{1}+\sum_{j=2}^{\ell_{1}} \delta_{n, j} \prod_{i=1}^{j-1}\left(1-\delta_{n, i}\right) \Gamma^{j-1} v_{n}^{1}+\prod_{i=1}^{\ell_{1}}\left(1-\delta_{n, i}\right) \Gamma^{\ell_{1}} v_{n}^{1}-q\right\|^{2} \\
\leq & \left\|t_{n+1}-q\right\|^{2}+\delta_{n, 1}\left\|v_{n, 1}^{1}-q\right\|^{2}+\sum_{j=2}^{\ell_{1}} \delta_{n, j} \prod_{i=1}^{j-1}\left(1-\delta_{n, i}\right)\left\|\Gamma^{j-1} v_{n}^{1}-\Gamma^{j-1} q\right\|^{2} \\
& +\prod_{i=1}^{\ell_{1}}\left(1-\delta_{n, i}\right)\left\|\Gamma^{\ell_{1}} v_{n}^{1}-\Gamma^{\ell_{1}} q\right\|^{2} \\
\leq & \left\|t_{n+1}-q\right\|^{2}+\delta_{n, 1}\left\|v_{n, 1}^{1}-q\right\|^{2}+\sum_{j=2}^{\ell_{1}} \delta_{n, j} \prod_{i=1}^{j-1}\left(1-\delta_{n, i}\right)\left(\rho^{j}\right)^{2}\left\|v_{n}^{1}-q\right\|^{2} \\
& +\prod_{i=1}^{\ell_{1}\left(1-\delta_{n, i}\right)\left(\rho^{j}\right)^{2}\left\|v_{n}^{1}-q\right\|^{2}}
\end{aligned}
$$




$$
\begin{aligned}
= & \left\|t_{n+1}-q\right\|^{2}+\left(\delta_{n, 1}+\sum_{j=2}^{\ell_{1}} \delta_{n, j} \prod_{i=1}^{j-1}\left(1-\delta_{n, i}\right)\left(\rho^{j}\right)^{2}+\prod_{i=1}^{\ell_{1}}\left(1-\delta_{n, i}\right)\left(\rho^{j}\right)^{2}\right) \\
& \times\left\|v_{n}^{1}-q\right\|^{2}
\end{aligned}
$$

Putting (4.6) into (4.9), and using (4.8), we get

$$
\begin{aligned}
\epsilon_{n} \leq & \left\|t_{n+1}-q\right\|^{2}+\left(\delta_{n, 1}+\sum_{j=2}^{\ell_{1}} \delta_{n, j} \prod_{i=1}^{j-1}\left(1-\delta_{n, i}\right)\left(\rho^{j}\right)^{2}+\prod_{i=1}^{\ell_{1}}\left(1-\delta_{n, i}\right)\left(\rho^{j}\right)^{2}\right) \\
& \times\left(\alpha_{n, 1}^{1}+\sum_{j=2}^{\ell_{2}} \alpha_{n, j}^{1}\left(\rho^{j}\right)^{2} \prod_{i=1}^{j-1}\left(1-\alpha_{n, i}^{1}\right)+\prod_{i=1}^{\ell_{2}}\left(1-\alpha_{n, i}^{1}\right)\left(\rho^{j}\right)^{2}\right) \\
& \times\left(\alpha_{n, 1}^{2}+\sum_{j=2}^{\ell_{3}} \alpha_{n, j}^{2}\left(\rho^{j}\right)^{2} \prod_{i=1}^{j-1}\left(1-\alpha_{n, i}\right)+\prod_{i=1}^{\ell_{3}}\left(1-\alpha_{n, i}^{2}\right)\left(\rho^{j}\right)^{2}\right) \\
& \times\left(\alpha_{n, 1}^{3}+\sum_{j=2}^{\ell_{4}} \alpha_{n, j}^{3}\left(\rho^{j}\right)^{2} \prod_{i=1}^{j-1}\left(1-\alpha_{n, i}^{3}\right)+\prod_{i=1}^{\ell_{4}}\left(1-\alpha_{n, i}^{3}\right)\left(\rho^{j}\right)^{2}\right) \\
& \times\left(\alpha_{n, 1}^{4}+\sum_{j=2}^{\ell_{5}} \alpha_{n, j}^{4}\left(\rho^{j}\right)^{2} \prod_{i=1}^{j-1}\left(1-\alpha_{n, i}^{4}\right)+\prod_{i=1}^{\ell_{5}}\left(1-\alpha_{n, i}^{4}\right)\left(\rho^{j}\right)^{2}\right) \\
& \times \cdots \times\left(\alpha_{n, 1}^{\ell_{s-2}}+\sum_{j=2}^{\ell_{s-1}} \alpha_{n, j}^{\ell_{s-2}}\left(\rho^{j}\right)^{2} \prod_{i=1}^{j-1}\left(1-\alpha_{n, i}^{\ell_{s-2}}\right)+\prod_{i=1}^{\ell_{s-1}}\left(1-\alpha_{n, i}^{\ell_{s-2}}\right)\left(\rho^{j}\right)^{2}\right) \\
\leq & \left\|t_{n+1}-q\right\|^{2}+\tau_{n}\left\|t_{n}-q\right\|^{2} \\
& \times\left(\alpha_{n, 1}^{\ell_{s-1}}+\sum_{j=2}^{\ell_{s}} \alpha_{n, j}^{\ell_{s-1}}\left(\rho^{j}\right)^{2} \prod_{i=1}^{j-1}\left(1-\alpha_{n, i}^{\ell_{s-1}}\right)+\prod_{i=1}^{\ell_{s}}\left(1-\alpha_{n, i}^{\ell_{s-1}}\right)\left(\rho^{j}\right)^{2}\right) \\
& \times t_{n}-q \|^{2} \\
& \ldots
\end{aligned}
$$

Thus, from our assumption, we obtain from (4.10) that $\epsilon_{n} \rightarrow 0$ as $n \rightarrow \infty$. Hence, the multistep $D /$-iteration scheme (3.2) is $\Gamma$-stable. Thus, tje proof is completed.

Theorem 4.2. Let $H$ be a Hilbert space, $\Gamma: H \longrightarrow H$ be a self-map of $H$ satisfying the contractive condition

$$
\left\|\Gamma^{j} x-\Gamma^{j} y\right\| \leq \rho^{j}\|x-y\|+\sum_{i=0}^{j}\left(\begin{array}{c}
j \\
i
\end{array}\right) \rho^{j-1} \phi(\|x-\Gamma x\|),
$$

where $x, y \in H, 0 \leq \rho^{j}<1$, and let $\phi$ retains its usual meaning with $\phi(0)=0$ and $\phi(M t)=$ $M \phi(t), M \geq 0, t \in \mathbb{R}^{+}$. For arbitrary $x_{0} \in H$, let $\left\{\omega_{n}\right\}_{n=0}^{\infty}$ be the multistep $/ H$-iteration scheme defined by (3.1). Assume $F(\Gamma) \neq \emptyset, q \in F(\Gamma)$. Then, the multisetp IH-iteration scheme is $\Gamma$-stable. 
Proof. Let $\left\{t_{n}\right\}_{n=0}^{\infty}$ and $\left\{v_{n}\right\}_{n=0}^{\infty}$, for $i=1,2, \cdots, s-1$, be two real sequences in $H$. Set

$$
\epsilon_{n}=\left\|t_{n+1}-\delta_{n, 1} t_{n}-\sum_{j=2}^{\ell_{1}} \delta_{n, j} \prod_{i=1}^{j-1}\left(1-\delta_{n, i}\right) \Gamma^{j-1} v_{n}^{1}-\prod_{i=1}^{\ell_{1}}\left(1-\delta_{n, i}\right) \Gamma^{\ell_{1}} v_{n}^{1}\right\|^{2}
$$

where, for $s=1,2, \cdots, k-2$,

$$
v_{n}^{s}=\alpha_{n, 1}^{s} t_{n}+\sum_{j=2}^{\ell_{s+1}} \alpha_{n, j}^{s} \prod_{i=1}^{j-1}\left(1-\alpha_{n, i}^{s}\right) \Gamma^{j-1} v_{n}^{s+1}+\prod_{i=1}^{\ell_{s+1}}\left(1-\alpha_{n, i}^{s}\right) \Gamma^{\ell_{1}} v_{n}^{s+1}
$$

and, for $k \geq 2$,

$$
v_{n}^{k-1}=\sum_{j=1}^{\ell_{k}} \alpha_{n, j}^{k-1} \prod_{i=1}^{j-1}\left(1-\alpha_{n, i}^{k-1}\right) \Gamma^{j-1} t_{n}+\prod_{i=1}^{\ell_{k}}\left(1-\alpha_{n, i}^{k-1}\right) \Gamma^{\ell_{k}} t_{n}, n \geq 1,
$$

Now, suppose $\epsilon_{n} \rightarrow 0$ as $n \rightarrow \infty$. Then, we show that $t_{n} \rightarrow q$ as $n \rightarrow \infty$ using contractive mapping defined by (4.1).

Indeed, Using Proposition 2.4 with $u=q, t_{n}=t, j=i, k=1, \Gamma^{j-1} v_{n}^{1}=v_{j-1}$ and $\Gamma^{\ell_{1}} v_{n}^{1}=v_{n}$ we obtain

$$
\begin{aligned}
& \left\|t_{n+1}-q\right\|^{2}=\| \delta_{n, 1} t_{n}+\sum_{j=2}^{\ell_{1}} \delta_{n, j} \prod_{i=1}^{j-1}\left(1-\delta_{n, i}\right) \Gamma^{j-1} v_{n}^{1}+\prod_{i=1}^{\ell_{1}}\left(1-\delta_{n, i}\right) \Gamma^{\ell_{1}} v_{n}^{1}-q \\
& -\left[\delta_{n, 1} t_{n}+\sum_{j=2}^{\ell_{1}} \delta_{n, j} \prod_{i=1}^{j-1}\left(1-\delta_{n, i}\right) \Gamma^{j-1} v_{n}^{1}+\prod_{i=1}^{\ell_{1}}\left(1-\delta_{n, i}\right) \Gamma^{\ell_{1}} v_{n}^{1}-t_{n+1}\right] \|^{2} \\
& \leq\left\|-\left[t_{n+1}-\delta_{n, 1} t_{n}-\sum_{j=2}^{\ell_{1}} \delta_{n, j} \prod_{i=1}^{j-1}\left(1-\delta_{n, i}\right) \Gamma^{j-1} v_{n}^{1}-\prod_{i=1}^{\ell_{1}}\left(1-\delta_{n, i}\right) \Gamma^{\ell_{1}} v_{n}^{1}\right]\right\|^{2} \\
& +\left\|\delta_{n, 1} t_{n}+\sum_{j=2}^{\ell_{1}} \delta_{n, j} \prod_{i=1}^{j-1}\left(1-\delta_{n, i}\right) \Gamma^{j-1} v_{n}^{1}+\prod_{i=1}^{\ell_{1}}\left(1-\delta_{n, i}\right) \Gamma^{\ell_{1}} v_{n}^{1}-q\right\|^{2} \\
& =\left\|t_{n+1}-\delta_{n, 1} t_{n}-\sum_{j=2}^{\ell_{1}} \delta_{n, j} \prod_{i=1}^{j-1}\left(1-\delta_{n, i}\right) \Gamma^{j-1} v_{n}^{1}-\prod_{i=1}^{\ell_{1}}\left(1-\delta_{n, i}\right) \Gamma^{\ell_{1}} v_{n}^{1}\right\|^{2} \\
& +\left\|\delta_{n, 1} t_{n}+\sum_{j=2}^{\ell_{1}} \delta_{n, j} \prod_{i=1}^{j-1}\left(1-\delta_{n, i}\right) \Gamma^{j-1} v_{n}^{1}+\prod_{i=1}^{\ell_{1}}\left(1-\delta_{n, i}\right) \Gamma^{\ell_{1}} v_{n}^{1}-q\right\|^{2} \\
& =\epsilon_{n}+\left\|\delta_{n, 1} t_{n}+\sum_{j=2}^{\ell_{1}} \delta_{n, j} \prod_{i=1}^{j-1}\left(1-\delta_{n, i}\right) \Gamma^{j-1} v_{n}^{1}+\prod_{i=1}^{\ell_{1}}\left(1-\delta_{n, i}\right) \Gamma^{\ell_{1}} v_{n}^{1}-q\right\|^{2} \\
& \leq \epsilon_{n}+\delta_{n, 1}\left\|t_{n}-q\right\|^{2}+\sum_{j=2}^{\ell_{1}} \delta_{n, j} \prod_{i=1}^{j-1}\left(1-\delta_{n, i}\right)\left\|\Gamma^{j-1} v_{n}^{1}-q\right\|^{2} \\
& +\prod_{i=1}^{\ell_{1}}\left(1-\delta_{n, i}\right)\left\|\Gamma^{\ell_{1}} v_{n}^{1}-q\right\|^{2}
\end{aligned}
$$




$$
\begin{aligned}
& \leq \epsilon_{n}+\delta_{n, 1}\left\|t_{n}-q\right\|^{2}+\sum_{j=2}^{\ell_{1}} \delta_{n, j}\left(\rho^{j}\right)^{2} \prod_{i=1}^{j-1}\left(1-\delta_{n, i}\right)\left\|v_{n}^{1}-q\right\|^{2} \\
&+\prod_{i=1}^{\ell_{1}}\left(1-\delta_{n, i}\right)\left(\rho^{j}\right)^{2}\left\|v_{n}^{1}-q\right\|^{2} \\
&= \epsilon_{n}+\delta_{n, 1}\left\|t_{n}-q\right\|^{2}+\left(1-\delta_{n, 1}-\prod_{i=1}^{\ell_{1}}\left(1-\delta_{n, i}\right)\right)\left(\rho^{j}\right)^{2}\left\|v_{n}^{1}-q\right\|^{2} \\
&+\prod_{i=1}^{\ell_{1}}\left(1-\delta_{n, i}\right)\left(\rho^{j}\right)^{2}\left\|v_{n}^{1}-q\right\|^{2} \\
&<\epsilon_{n}+\delta_{n, 1}\left\|t_{n}-q\right\|^{2}+\left(1-\delta_{n, 1}\right)\left\|v_{n}^{1}-q\right\|^{2}
\end{aligned}
$$

Since $\ell_{1}, \ell_{k}$ are fixed integers and $\alpha_{n, i}^{s} \in[0,1]$ for each $s$, the estimations below are obtained for $n=1,2, \cdots$ and $1 \leq s \leq k-1$ :

$$
\begin{aligned}
& \left\|v_{n}^{1}-q\right\|^{2} \leq \alpha_{n, 1}\left\|t_{n}-q\right\|^{2}+\sum_{j=2}^{\ell_{2}} \alpha_{n, j} \prod_{i=1}^{j-1}\left(1-\alpha_{n, i}\right)\left\|\Gamma^{j-1} v_{n}^{2}-\Gamma^{j-1} q\right\|^{2} \\
& +\prod_{i=1}^{\ell_{2}}\left(1-\alpha_{n, i}\right)\left\|\Gamma^{\ell_{2}} v_{n}^{2}-\Gamma^{\ell_{2}} q\right\|^{2} \\
& \leq \alpha_{n, 1}^{1}\left\|t_{n}-q\right\|^{2}+\sum_{j=2}^{\ell_{2}} \alpha_{n, j}\left(\rho^{j}\right)^{2} \prod_{i=1}^{j-1}\left(1-\alpha_{n, i}\right)\left\|v_{n}^{2}-q\right\|^{2} \\
& +\prod_{i=1}^{\ell_{2}}\left(1-\alpha_{n, i}\right)\left(\rho^{j}\right)^{2}\left\|v_{n}^{2}-q\right\|^{2} \\
& \leq \alpha_{n, 1}^{1}\left\|t_{n}-q\right\|^{2}+\sum_{j=2}^{\ell_{2}} \alpha_{n, j}^{1}\left(\rho^{j}\right)^{2} \prod_{i=1}^{j-1}\left(1-\alpha_{n, i}^{1}\right)\left[\alpha_{n, 1}^{2}\left\|t_{n}-q\right\|^{2}\right. \\
& \left.+\sum_{j=2}^{\ell_{3}} \alpha_{n, j}^{2}\left(\rho^{j}\right)^{2} \prod_{i=1}^{j-1}\left(1-\alpha_{n, i}^{2}\right)\left\|v_{n}^{3}-q\right\|^{2}+\prod_{i=1}^{\ell_{3}}\left(1-\alpha_{n, i}^{2}\right)\left(\rho^{j}\right)^{2}\left\|v_{n}^{3}-q\right\|^{2}\right] \\
& +\prod_{i=1}^{\ell_{2}}\left(1-\alpha_{n, i}^{1}\right)\left(\rho^{j}\right)^{2}\left[\alpha_{n, 1}^{2}\left\|t_{n}-q\right\|^{2}+\sum_{j=2}^{\ell_{3}} \alpha_{n, j}^{2}\left(\rho^{j}\right)^{2} \prod_{i=1}^{j-1}\left(1-\alpha_{n, i}^{2}\right)\left\|v_{n}^{3}-q\right\|^{2}\right. \\
& \left.+\prod_{i=1}^{\ell_{3}}\left(1-\alpha_{n, i}^{2}\right)\left(\rho^{j}\right)^{2}\left\|v_{n}^{3}-q\right\|^{2}\right] \\
& =\alpha_{n, 1}^{1}\left\|t_{n}-q\right\|^{2}+\sum_{j=2}^{\ell_{2}} \alpha_{n, j}^{1}\left(\rho^{j}\right)^{2} \prod_{i=1}^{j-1}\left(1-\alpha_{n, i}^{1}\right) \alpha_{n, 1}^{2}\left\|t_{n}-q\right\|^{2} \\
& +\left(\sum_{j=2}^{\ell_{2}} \alpha_{n, j}^{1}\left(\rho^{j}\right)^{2} \prod_{i=1}^{j-1}\left(1-\alpha_{n, i}^{1}\right)\right)\left(\sum_{j=2}^{\ell_{3}} \alpha_{n, j}^{2}\left(\rho^{j}\right)^{2} \prod_{i=1}^{j-1}\left(1-\alpha_{n, i}^{2}\right)\right)\left\|v_{n}^{3}-q\right\|^{2}
\end{aligned}
$$




$$
\begin{aligned}
& +\left(\sum_{j=2}^{\ell_{2}} \alpha_{n, j}^{1}\left(\rho^{j}\right)^{2} \prod_{i=1}^{j-1}\left(1-\alpha_{n, i}^{1}\right)\right)\left(\prod_{i=1}^{\ell_{3}}\left(1-\alpha_{n, i}^{2}\right)\left(\rho^{j}\right)^{2}\right)\left\|v_{n}^{3}-q\right\|^{2} \\
& +\prod_{i=1}^{\ell_{2}}\left(1-\alpha_{n, i}^{1}\right)\left(\rho^{j}\right)^{2} \alpha_{n, 1}^{2}\left\|t_{n}-q\right\|^{2} \\
& +\left(\sum_{j=2}^{\ell_{3}} \alpha_{n, j}^{2}\left(\rho^{j}\right)^{2} \prod_{i=1}^{j-1}\left(1-\alpha_{n, i}^{2}\right)\right)\left(\prod_{i=1}^{\ell_{2}}\left(1-\alpha_{n, i}^{1}\right)\left(\rho^{j}\right)^{2}\right)\left\|v_{n}^{3}-q\right\|^{2} \\
& +\left(\prod_{i=1}^{\ell_{3}}\left(1-\alpha_{n, i}^{2}\right)\left(\rho^{j}\right)^{2}\right)\left(\prod_{i=1}^{\ell_{2}}\left(1-\alpha_{n, i}^{1}\right)\left(\rho^{j}\right)^{2}\right)\left\|v_{n}^{3}-q\right\|^{2} \\
& \leq \alpha_{n, 1}^{1}\left\|t_{n}-q\right\|^{2}+\sum_{j=2}^{\ell_{2}} \alpha_{n, j}^{1}\left(\rho^{j}\right)^{2} \prod_{i=1}^{j-1}\left(1-\alpha_{n, i}^{1}\right) \alpha_{n, 1}^{2}\left\|t_{n}-q\right\|^{2} \\
& +\prod_{i=1}^{\ell_{2}}\left(1-\alpha_{n, i}^{1}\right)\left(\rho^{j}\right)^{2} \alpha_{n, 1}^{2}\left\|t_{n}-q\right\|^{2} \\
& +\left(\sum_{j=2}^{\ell_{2}} \alpha_{n, j}^{1}\left(\rho^{j}\right)^{2} \prod_{i=1}^{j-1}\left(1-\alpha_{n, i}^{1}\right)\right)\left(\sum_{j=2}^{\ell_{3}} \alpha_{n, j}^{2}\left(\rho^{j}\right)^{2} \prod_{i=1}^{j-1}\left(1-\alpha_{n, i}^{2}\right)\right)\left[\alpha_{n, 1}^{3}\left\|t_{n}-q\right\|^{2}\right. \\
& \left.+\sum_{j=2}^{\ell_{4}} \alpha_{n, j}^{3}\left(\rho^{j}\right)^{2} \prod_{i=1}^{j-1}\left(1-\alpha_{n, i}^{3}\right)\left\|v_{n}^{4}-q\right\|^{2}+\prod_{i=1}^{\ell_{4}}\left(1-\alpha_{n, i}^{4}\right)\left(\rho^{j}\right)^{2}\left\|v_{n}^{4}-q\right\|^{2}\right] \\
& +\left(\sum_{j=2}^{\ell_{2}} \alpha_{n, j}^{1}\left(\rho^{j}\right)^{2} \prod_{i=1}^{j-1}\left(1-\alpha_{n, i}^{1}\right)\right)\left(\prod_{i=1}^{\ell_{3}}\left(1-\alpha_{n, i}^{2}\right)\left(\rho^{j}\right)^{2}\right)\left[\alpha_{n, 1}^{3}\left\|t_{n}-q\right\|^{2}\right. \\
& \left.+\sum_{j=2}^{\ell_{4}} \alpha_{n, j}^{3}\left(\rho^{j}\right)^{2} \prod_{i=1}^{j-1}\left(1-\alpha_{n, i}^{3}\right)\left\|v_{n}^{4}-q\right\|^{2}+\prod_{i=1}^{\ell_{4}}\left(1-\alpha_{n, i}^{4}\right)\left(\rho^{j}\right)^{2}\left\|v_{n}^{4}-q\right\|^{2}\right] \\
& +\left(\sum_{j=2}^{\ell_{3}} \alpha_{n, j}^{2}\left(\rho^{j}\right)^{2} \prod_{i=1}^{j-1}\left(1-\alpha_{n, i}^{2}\right)\right)\left(\prod_{i=1}^{\ell_{2}}\left(1-\alpha_{n, i}^{1}\right)\left(\rho^{j}\right)^{2}\right)\left[\alpha_{n, 1}^{3}\left\|t_{n}-q\right\|^{2}\right. \\
& \left.+\sum_{j=2}^{\ell_{4}} \alpha_{n, j}^{3}\left(\rho^{j}\right)^{2} \prod_{i=1}^{j-1}\left(1-\alpha_{n, i}^{3}\right)\left\|v_{n}^{4}-q\right\|^{2}+\prod_{i=1}^{\ell_{4}}\left(1-\alpha_{n, i}^{4}\right)\left(\rho^{j}\right)^{2}\left\|v_{n}^{4}-q\right\|^{2}\right] \\
& +\left(\prod_{i=1}^{\ell_{3}}\left(1-\alpha_{n, i}^{2}\right)\left(\rho^{j}\right)^{2}\right)\left(\prod_{i=1}^{\ell_{2}}\left(1-\alpha_{n, i}^{1}\right)\left(\rho^{j}\right)^{2}\right)\left[\alpha_{n, 1}^{3}\left\|t_{n}-q\right\|^{2}\right. \\
& \left.+\sum_{j=2}^{\ell_{4}} \alpha_{n, j}^{3}\left(\rho^{j}\right)^{2} \prod_{i=1}^{j-1}\left(1-\alpha_{n, i}^{3}\right)\left\|v_{n}^{4}-q\right\|^{2}+\prod_{i=1}^{\ell_{4}}\left(1-\alpha_{n, i}^{4}\right)\left(\rho^{j}\right)^{2}\left\|v_{n}^{4}-q\right\|^{2}\right] \\
& =\alpha_{n, 1}^{1}\left\|t_{n}-q\right\|^{2}+\sum_{j=2}^{\ell_{2}} \alpha_{n, j}^{1}\left(\rho^{j}\right)^{2} \prod_{i=1}^{j-1}\left(1-\alpha_{n, i}^{1}\right) \alpha_{n, 1}^{2}\left\|t_{n}-q\right\|^{2} \\
& +\prod_{i=1}^{\ell_{2}}\left(1-\alpha_{n, i}^{1}\right)\left(\rho^{j}\right)^{2} \alpha_{n, 1}^{2}\left\|t_{n}-q\right\|^{2}
\end{aligned}
$$




$$
\begin{aligned}
& +\left(\sum_{j=2}^{\ell_{2}} \alpha_{n, j}^{1}\left(\rho^{j}\right)^{2} \prod_{i=1}^{j-1}\left(1-\alpha_{n, i}^{1}\right)\right)\left(\sum_{j=2}^{\ell_{3}} \alpha_{n, j}^{2}\left(\rho^{j}\right)^{2} \prod_{i=1}^{j-1}\left(1-\alpha_{n, i}^{2}\right)\right) \alpha_{n, 1}^{3}\left\|t_{n}-q\right\|^{2} \\
& +\left(\sum_{j=2}^{\ell_{2}} \alpha_{n, j}^{1}\left(\rho^{j}\right)^{2} \prod_{i=1}^{j-1}\left(1-\alpha_{n, i}^{1}\right)\right)\left(\sum_{j=2}^{\ell_{3}} \alpha_{n, j}^{2}\left(\rho^{j}\right)^{2} \prod_{i=1}^{j-1}\left(1-\alpha_{n, i}^{2}\right)\right) \\
& \times\left(\sum_{j=2}^{\ell_{4}} \alpha_{n, j}^{3}\left(\rho^{j}\right)^{2} \prod_{i=1}^{j-1}\left(1-\alpha_{n, i}^{3}\right)\right)\left\|v_{n}^{4}-q\right\|^{2}+\left(\sum_{j=2}^{\ell_{2}} \alpha_{n, j}^{1}\left(\rho^{j}\right)^{2} \prod_{i=1}^{j-1}\left(1-\alpha_{n, i}^{1}\right)\right) \\
& \times\left(\sum_{j=2}^{\ell_{3}} \alpha_{n, j}^{2}\left(\rho^{j}\right)^{2} \prod_{i=1}^{j-1}\left(1-\alpha_{n, i}^{2}\right)\right)\left(\prod_{i=1}^{\ell_{4}}\left(1-\alpha_{n, i}^{4}\right)\left(\rho^{j}\right)^{2}\right)\left\|v_{n}^{4}-q\right\|^{2} \\
& +\left(\sum_{j=2}^{\ell_{2}} \alpha_{n, j}^{1}\left(\rho^{j}\right)^{2} \prod_{i=1}^{j-1}\left(1-\alpha_{n, i}^{1}\right)\right)\left(\prod_{i=1}^{\ell_{3}}\left(1-\alpha_{n, i}^{2}\right)\left(\rho^{j}\right)^{2}\right)\left\|t_{n}-q\right\|^{2} \\
& +\left(\sum_{j=2}^{\ell_{2}} \alpha_{n, j}^{1}\left(\rho^{j}\right)^{2} \prod_{i=1}^{j-1}\left(1-\alpha_{n, i}^{1}\right)\right)\left(\prod_{i=1}^{\ell_{3}}\left(1-\alpha_{n, i}^{2}\right)\left(\rho^{j}\right)^{2}\right)\left(\sum_{j=2}^{\ell_{4}} \alpha_{n, j}^{3}\left(\rho^{j}\right)^{2} \prod_{i=1}^{j-1}\left(1-\alpha_{n, i}^{3}\right)\right)\left\|v_{n}^{4}-q\right\|^{2} \\
& +\left(\sum_{j=2}^{\ell_{2}} \alpha_{n, j}^{1}\left(\rho^{j}\right)^{2} \prod_{i=1}^{j-1}\left(1-\alpha_{n, i}^{1}\right)\right)\left(\prod_{i=1}^{\ell_{3}}\left(1-\alpha_{n, i}^{2}\right)\left(\rho^{j}\right)^{2}\right)\left(\prod_{i=1}^{\ell_{4}}\left(1-\alpha_{n, i}^{4}\right)\left(\rho^{j}\right)^{2}\right)\left\|v_{n}^{4}-q\right\|^{2} \\
& +\left(\sum_{j=2}^{\ell_{3}} \alpha_{n, j}^{2}\left(\rho^{j}\right)^{2} \prod_{i=1}^{j-1}\left(1-\alpha_{n, i}^{2}\right)\right)\left(\prod_{i=1}^{\ell_{2}}\left(1-\alpha_{n, i}^{1}\right)\left(\rho^{j}\right)^{2}\right) \alpha_{n, 1}^{3}\left\|t_{n}-q\right\|^{2} \\
& +\left(\sum_{j=2}^{\ell_{3}} \alpha_{n, j}^{2}\left(\rho^{j}\right)^{2} \prod_{i=1}^{j-1}\left(1-\alpha_{n, i}^{2}\right)\right)\left(\prod_{i=1}^{\ell_{2}}\left(1-\alpha_{n, i}^{1}\right)\left(\rho^{j}\right)^{2}\right)\left(\sum_{j=2}^{\ell_{4}} \alpha_{n, j}^{3}\left(\rho^{j}\right)^{2} \prod_{i=1}^{j-1}\left(1-\alpha_{n, i}^{3}\right)\right)\left\|v_{n}^{4}-q\right\|^{2} \\
& +\left(\sum_{j=2}^{\ell_{3}} \alpha_{n, j}^{2}\left(\rho^{j}\right)^{2} \prod_{i=1}^{j-1}\left(1-\alpha_{n, i}^{2}\right)\right)\left(\prod_{i=1}^{\ell_{2}}\left(1-\alpha_{n, i}^{1}\right)\left(\rho^{j}\right)^{2}\right)\left(\prod_{i=1}^{\ell_{4}}\left(1-\alpha_{n, i}^{4}\right)\left(\rho^{j}\right)^{2}\right)\left\|v_{n}^{4}-q\right\|^{2} \\
& +\left(\prod_{i=1}^{\ell_{3}}\left(1-\alpha_{n, i}^{2}\right)\left(\rho^{j}\right)^{2}\right)\left(\prod_{i=1}^{\ell_{2}}\left(1-\alpha_{n, i}^{1}\right)\left(\rho^{j}\right)^{2}\right) \alpha_{n, 1}^{3}\left\|t_{n}-q\right\|^{2} \\
& +\left(\prod_{i=1}^{\ell_{3}}\left(1-\alpha_{n, i}^{2}\right)\left(\rho^{j}\right)^{2}\right)\left(\prod_{i=1}^{\ell_{2}}\left(1-\alpha_{n, i}^{1}\right)\left(\rho^{j}\right)^{2}\right)\left(\sum_{j=2}^{\ell_{4}} \alpha_{n, j}^{3}\left(\rho^{j}\right)^{2} \prod_{i=1}^{j-1}\left(1-\alpha_{n, i}^{3}\right)\right)\left\|v_{n}^{4}-q\right\|^{2} \\
& +\left(\prod_{i=1}^{\ell_{3}}\left(1-\alpha_{n, i}^{2}\right)\left(\rho^{j}\right)^{2}\right)\left(\prod_{i=1}^{\ell_{2}}\left(1-\alpha_{n, i}^{1}\right)\left(\rho^{j}\right)^{2}\right)\left(\prod_{i=1}^{\ell_{4}}\left(1-\alpha_{n, i}^{4}\right)\left(\rho^{j}\right)^{2}\right)\left\|v_{n}^{4}-q\right\|^{2} \\
& =\alpha_{n, 1}^{1}\left\|t_{n}-q\right\|^{2}+\sum_{j=2}^{\ell_{2}} \alpha_{n, j}^{1}\left(\rho^{j}\right)^{2} \prod_{i=1}^{j-1}\left(1-\alpha_{n, i}^{1}\right) \alpha_{n, 1}^{2}\left\|t_{n}-q\right\|^{2} \\
& +\prod_{i=1}^{\ell_{2}}\left(1-\alpha_{n, i}^{1}\right)\left(\rho^{j}\right)^{2} \alpha_{n, 1}^{2}\left\|t_{n}-q\right\|^{2}
\end{aligned}
$$




$$
\begin{aligned}
& +\left(\sum_{j=2}^{\ell_{2}} \alpha_{n, j}^{1}\left(\rho^{j}\right)^{2} \prod_{i=1}^{j-1}\left(1-\alpha_{n, i}^{1}\right)\right)\left(\sum_{j=2}^{\ell_{3}} \alpha_{n, j}^{2}\left(\rho^{j}\right)^{2} \prod_{i=1}^{j-1}\left(1-\alpha_{n, i}^{2}\right)\right) \alpha_{n, 1}^{3}\left\|t_{n}-q\right\|^{2} \\
& +\left(\sum_{j=2}^{\ell_{2}} \alpha_{n, j}^{1}\left(\rho^{j}\right)^{2} \prod_{i=1}^{j-1}\left(1-\alpha_{n, i}^{1}\right)\right)\left(\prod_{i=1}^{\ell_{3}}\left(1-\alpha_{n, i}^{2}\right)\left(\rho^{j}\right)^{2}\right)\left\|t_{n}-q\right\|^{2} \\
& +\left(\sum_{j=2}^{\ell_{3}} \alpha_{n, j}^{2}\left(\rho^{j}\right)^{2} \prod_{i=1}^{j-1}\left(1-\alpha_{n, i}^{2}\right)\right)\left(\prod_{i=1}^{\ell_{2}}\left(1-\alpha_{n, i}^{1}\right)\left(\rho^{j}\right)^{2}\right) \alpha_{n, 1}^{3}\left\|t_{n}-q\right\|^{2} \\
& +\left(\prod_{i=1}^{\ell_{3}}\left(1-\alpha_{n, i}^{2}\right)\left(\rho^{j}\right)^{2}\right)\left(\prod_{i=1}^{\ell_{2}}\left(1-\alpha_{n, i}^{1}\right)\left(\rho^{j}\right)^{2}\right) \alpha_{n, 1}^{3}\left\|t_{n}-q\right\|^{2} \\
& +\left(\sum_{j=2}^{\ell_{2}} \alpha_{n, j}^{1}\left(\rho^{j}\right)^{2} \prod_{i=1}^{j-1}\left(1-\alpha_{n, i}^{1}\right)\right)\left(\sum_{j=2}^{\ell_{3}} \alpha_{n, j}^{2}\left(\rho^{j}\right)^{2} \prod_{i=1}^{j-1}\left(1-\alpha_{n, i}^{2}\right)\right) \\
& \times\left(\left(1-\alpha_{n, 1}^{3}-\prod_{i=1}^{\ell_{4}}\left(1-\alpha_{n, i}^{3}\right)\right)\left(\rho^{j}\right)^{2}\right)\left\|v_{n}^{4}-q\right\|^{2}+\left(\sum_{j=2}^{\ell_{2}} \alpha_{n, j}^{1}\left(\rho^{j}\right)^{2} \prod_{i=1}^{j-1}\left(1-\alpha_{n, i}^{1}\right)\right) \\
& \times\left(\sum_{j=2}^{\ell_{3}} \alpha_{n, j}^{2}\left(\rho^{j}\right)^{2} \prod_{i=1}^{j-1}\left(1-\alpha_{n, i}^{2}\right)\right)\left(\prod_{i=1}^{\ell_{4}}\left(1-\alpha_{n, i}^{4}\right)\left(\rho^{j}\right)^{2}\right)\left\|v_{n}^{4}-q\right\|^{2} \\
& +\left(\sum_{j=2}^{\ell_{2}} \alpha_{n, j}^{1}\left(\rho^{j}\right)^{2} \prod_{i=1}^{j-1}\left(1-\alpha_{n, i}^{1}\right)\right)\left(\prod_{i=1}^{\ell_{3}}\left(1-\alpha_{n, i}^{2}\right)\left(\rho^{j}\right)^{2}\right) \\
& \left.\times\left(\left(1-\alpha_{n, 1}^{3}-\prod_{i=1}^{\ell_{4}}\left(1-\alpha_{n, i}^{3}\right)\right)\left(\rho^{j}\right)^{2}\right)\right)\left\|v_{n}^{4}-q\right\|^{2} \\
& +\left(\sum_{j=2}^{\ell_{2}} \alpha_{n, j}^{1}\left(\rho^{j}\right)^{2} \prod_{i=1}^{j-1}\left(1-\alpha_{n, i}^{1}\right)\right)\left(\prod_{i=1}^{\ell_{3}}\left(1-\alpha_{n, i}^{2}\right)\left(\rho^{j}\right)^{2}\right)\left(\prod_{i=1}^{\ell_{4}}\left(1-\alpha_{n, i}^{4}\right)\left(\rho^{j}\right)^{2}\right)\left\|v_{n}^{4}-q\right\|^{2} \\
& +\left(\sum_{j=2}^{\ell_{3}} \alpha_{n, j}^{2}\left(\rho^{j}\right)^{2} \prod_{i=1}^{j-1}\left(1-\alpha_{n, i}^{2}\right)\right)\left(\prod_{i=1}^{\ell_{2}}\left(1-\alpha_{n, i}^{1}\right)\left(\rho^{j}\right)^{2}\right) \\
& \left.\times\left(\left(1-\alpha_{n, 1}^{3}-\prod_{i=1}^{\ell_{4}}\left(1-\alpha_{n, i}^{3}\right)\right)\left(\rho^{j}\right)^{2}\right)\right)\left\|v_{n}^{4}-q\right\|^{2} \\
& +\left(\sum_{j=2}^{\ell_{3}} \alpha_{n, j}^{2}\left(\rho^{j}\right)^{2} \prod_{i=1}^{j-1}\left(1-\alpha_{n, i}^{2}\right)\right)\left(\prod_{i=1}^{\ell_{2}}\left(1-\alpha_{n, i}^{1}\right)\left(\rho^{j}\right)^{2}\right)\left(\prod_{i=1}^{\ell_{4}}\left(1-\alpha_{n, i}^{4}\right)\left(\rho^{j}\right)^{2}\right)\left\|v_{n}^{4}-q\right\|^{2} \\
& +\left(\prod_{i=1}^{\ell_{3}}\left(1-\alpha_{n, i}^{2}\right)\left(\rho^{j}\right)^{2}\right)\left(\prod_{i=1}^{\ell_{2}}\left(1-\alpha_{n, i}^{1}\right)\left(\rho^{j}\right)^{2}\right)\left(\left(1-\alpha_{n, 1}^{3}-\prod_{i=1}^{\ell_{4}}\left(1-\alpha_{n, i}^{3}\right)\right)\left(\rho^{j}\right)^{2}\right)\left\|v_{n}^{4}-q\right\|^{2} \\
& +\left(\prod_{i=1}^{\ell_{3}}\left(1-\alpha_{n, i}^{2}\right)\left(\rho^{j}\right)^{2}\right)\left(\prod_{i=1}^{\ell_{2}}\left(1-\alpha_{n, i}^{1}\right)\left(\rho^{j}\right)^{2}\right)\left(\prod_{i=1}^{\ell_{4}}\left(1-\alpha_{n, i}^{4}\right)\left(\rho^{j}\right)^{2}\right)\left\|v_{n}^{4}-q\right\|^{2}
\end{aligned}
$$




$$
\begin{aligned}
& =\alpha_{n, 1}^{1}\left\|t_{n}-q\right\|^{2}+\sum_{j=2}^{\ell_{2}} \alpha_{n, j}^{1}\left(\rho^{j}\right)^{2} \prod_{i=1}^{j-1}\left(1-\alpha_{n, i}^{1}\right) \alpha_{n, 1}^{2}\left\|t_{n}-q\right\|^{2} \\
& +\prod_{i=1}^{\ell_{2}}\left(1-\alpha_{n, i}^{1}\right)\left(\rho^{j}\right)^{2} \alpha_{n, 1}^{2}\left\|t_{n}-q\right\|^{2} \\
& +\left(\sum_{j=2}^{\ell_{2}} \alpha_{n, j}^{1}\left(\rho^{j}\right)^{2} \prod_{i=1}^{j-1}\left(1-\alpha_{n, i}^{1}\right)\right)\left(\sum_{j=2}^{\ell_{3}} \alpha_{n, j}^{2}\left(\rho^{j}\right)^{2} \prod_{i=1}^{j-1}\left(1-\alpha_{n, i}^{2}\right)\right) \alpha_{n, 1}^{3}\left\|t_{n}-q\right\|^{2} \\
& +\left(\sum_{j=2}^{\ell_{2}} \alpha_{n, j}^{1}\left(\rho^{j}\right)^{2} \prod_{i=1}^{j-1}\left(1-\alpha_{n, i}^{1}\right)\right)\left(\prod_{i=1}^{\ell_{3}}\left(1-\alpha_{n, i}^{2}\right)\left(\rho^{j}\right)^{2}\right)\left\|t_{n}-q\right\|^{2} \\
& +\left(\sum_{j=2}^{\ell_{3}} \alpha_{n, j}^{2}\left(\rho^{j}\right)^{2} \prod_{i=1}^{j-1}\left(1-\alpha_{n, i}^{2}\right)\right)\left(\prod_{i=1}^{\ell_{2}}\left(1-\alpha_{n, i}^{1}\right)\left(\rho^{j}\right)^{2}\right) \alpha_{n, 1}^{3}\left\|t_{n}-q\right\|^{2} \\
& +\left(\prod_{i=1}^{\ell_{3}}\left(1-\alpha_{n, i}^{2}\right)\left(\rho^{j}\right)^{2}\right)\left(\prod_{i=1}^{\ell_{2}}\left(1-\alpha_{n, i}^{1}\right)\left(\rho^{j}\right)^{2}\right) \alpha_{n, 1}^{3}\left\|t_{n}-q\right\|^{2} \\
& +\left(\sum_{j=2}^{\ell_{2}} \alpha_{n, j}^{1}\left(\rho^{j}\right)^{2} \prod_{i=1}^{j-1}\left(1-\alpha_{n, i}^{1}\right)\right)\left(\sum_{j=2}^{\ell_{3}} \alpha_{n, j}^{2}\left(\rho^{j}\right)^{2} \prod_{i=1}^{j-1}\left(1-\alpha_{n, i}^{2}\right)\right)\left(1-\alpha_{n, 1}^{3}\right)\left(\rho^{j}\right)^{2}\left\|v_{n}^{4}-q\right\|^{2} \\
& \left.+\left(\sum_{j=2}^{\ell_{2}} \alpha_{n, j}^{1}\left(\rho^{j}\right)^{2} \prod_{i=1}^{j-1}\left(1-\alpha_{n, i}^{1}\right)\right)\left(\prod_{i=1}^{\ell_{3}}\left(1-\alpha_{n, i}^{2}\right)\left(\rho^{j}\right)^{2}\right)\left(1-\alpha_{n, 1}^{3}\right)\left(\rho^{j}\right)^{2}\right)\left\|v_{n}^{4}-q\right\|^{2} \\
& \left.+\left(\sum_{j=2}^{\ell_{3}} \alpha_{n, j}^{2}\left(\rho^{j}\right)^{2} \prod_{i=1}^{j-1}\left(1-\alpha_{n, i}^{2}\right)\right)\left(\prod_{i=1}^{\ell_{2}}\left(1-\alpha_{n, i}^{1}\right)\left(\rho^{j}\right)^{2}\right)\left(1-\alpha_{n, 1}^{3}\right)\left(\rho^{j}\right)^{2}\right)\left\|v_{n}^{4}-q\right\|^{2} \\
& +\left(\prod_{i=1}^{\ell_{3}}\left(1-\alpha_{n, i}^{2}\right)\left(\rho^{j}\right)^{2}\right)\left(\prod_{i=1}^{\ell_{2}}\left(1-\alpha_{n, i}^{1}\right)\left(\rho^{j}\right)^{2}\right)\left(1-\alpha_{n, 1}^{3}\right)\left(\rho^{j}\right)^{2}\left\|v_{n}^{4}-q\right\|^{2} \\
& \leq \alpha_{n, 1}^{1}\left\|t_{n}-q\right\|^{2}+\sum_{j=2}^{\ell_{2}} \alpha_{n, j}^{1}\left(\rho^{j}\right)^{2} \prod_{i=1}^{j-1}\left(1-\alpha_{n, i}^{1}\right) \alpha_{n, 1}^{2}\left\|t_{n}-q\right\|^{2} \\
& +\prod_{i=1}^{\ell_{2}}\left(1-\alpha_{n, i}^{1}\right)\left(\rho^{j}\right)^{2} \alpha_{n, 1}^{2}\left\|t_{n}-q\right\|^{2} \\
& +\left(\sum_{j=2}^{\ell_{2}} \alpha_{n, j}^{1}\left(\rho^{j}\right)^{2} \prod_{i=1}^{j-1}\left(1-\alpha_{n, i}^{1}\right)\right)\left(\sum_{j=2}^{\ell_{3}} \alpha_{n, j}^{2}\left(\rho^{j}\right)^{2} \prod_{i=1}^{j-1}\left(1-\alpha_{n, i}^{2}\right)\right) \alpha_{n, 1}^{3}\left\|t_{n}-q\right\|^{2} \\
& +\left(\sum_{j=2}^{\ell_{2}} \alpha_{n, j}^{1}\left(\rho^{j}\right)^{2} \prod_{i=1}^{j-1}\left(1-\alpha_{n, i}^{1}\right)\right)\left(\prod_{i=1}^{\ell_{3}}\left(1-\alpha_{n, i}^{2}\right)\left(\rho^{j}\right)^{2}\right)\left\|t_{n}-q\right\|^{2} \\
& +\left(\sum_{j=2}^{\ell_{3}} \alpha_{n, j}^{2}\left(\rho^{j}\right)^{2} \prod_{i=1}^{j-1}\left(1-\alpha_{n, i}^{2}\right)\right)\left(\prod_{i=1}^{\ell_{2}}\left(1-\alpha_{n, i}^{1}\right)\left(\rho^{j}\right)^{2}\right) \alpha_{n, 1}^{3}\left\|t_{n}-q\right\|^{2}
\end{aligned}
$$




$$
\begin{aligned}
& +\left(\prod_{i=1}^{\ell_{3}}\left(1-\alpha_{n, i}^{2}\right)\left(\rho^{j}\right)^{2}\right)\left(\prod_{i=1}^{\ell_{2}}\left(1-\alpha_{n, i}^{1}\right)\left(\rho^{j}\right)^{2}\right) \alpha_{n, 1}^{3}\left\|t_{n}-q\right\|^{2} \\
& +\left(\sum_{j=2}^{\ell_{2}} \alpha_{n, j}^{1}\left(\rho^{j}\right)^{2} \prod_{i=1}^{j-1}\left(1-\alpha_{n, i}^{1}\right)\right)\left(\sum_{j=2}^{\ell_{3}} \alpha_{n, j}^{2}\left(\rho^{j}\right)^{2} \prod_{i=1}^{j-1}\left(1-\alpha_{n, i}^{2}\right)\right) \alpha_{n, 1}^{3}\left(1-\alpha_{n, 1}^{3}\right)\left(\rho^{j}\right)^{2}\left\|t_{n}-q\right\|^{2} \\
& \left.+\left(\sum_{j=2}^{\ell_{2}} \alpha_{n, j}^{1}\left(\rho^{j}\right)^{2} \prod_{i=1}^{j-1}\left(1-\alpha_{n, i}^{1}\right)\right)\left(\prod_{i=1}^{\ell_{3}}\left(1-\alpha_{n, i}^{2}\right)\left(\rho^{j}\right)^{2}\right) \alpha_{n, 1}^{3}\left(1-\alpha_{n, 1}^{3}\right)\left(\rho^{j}\right)^{2}\right)\left\|t_{n}-q\right\|^{2} \\
& \left.+\left(\sum_{j=2}^{\ell_{3}} \alpha_{n, j}^{2}\left(\rho^{j}\right)^{2} \prod_{i=1}^{j-1}\left(1-\alpha_{n, i}^{2}\right)\right)\left(\prod_{i=1}^{\ell_{2}}\left(1-\alpha_{n, i}^{1}\right)\left(\rho^{j}\right)^{2}\right) \alpha_{n, 1}^{3}\left(1-\alpha_{n, 1}^{3}\right)\left(\rho^{j}\right)^{2}\right)\left\|t_{n}-q\right\|^{2} \\
& +\left(\prod_{i=1}^{\ell_{3}}\left(1-\alpha_{n, i}^{2}\right)\left(\rho^{j}\right)^{2}\right)\left(\prod_{i=1}^{\ell_{2}}\left(1-\alpha_{n, i}^{1}\right)\left(\rho^{j}\right)^{2}\right) \alpha_{n, 1}^{3}\left(1-\alpha_{n, 1}^{3}\right)\left(\rho^{j}\right)^{2}\left\|t_{n}-q\right\|^{2}+\cdots \\
& +\left(\sum_{j=2}^{\ell_{2}} \alpha_{n, j}^{1}\left(\rho^{j}\right)^{2} \prod_{i=1}^{j-1}\left(1-\alpha_{n, i}^{1}\right)\right)\left(\sum_{j=2}^{\ell_{3}} \alpha_{n, j}^{2}\left(\rho^{j}\right)^{2} \prod_{i=1}^{j-1}\left(1-\alpha_{n, i}^{2}\right)\right) \\
& \times\left(\sum_{j=2}^{\ell_{4}} \alpha_{n, j}^{3}\left(\rho^{j}\right)^{3} \prod_{i=1}^{j-1}\left(1-\alpha_{n, i}^{2}\right)\right) \times \cdots \times\left(\sum_{j=2}^{\ell_{s-1}} \alpha_{n, j}^{\ell_{s-2}}\left(\rho^{j}\right)^{2} \prod_{i=1}^{j-1}\left(1-\alpha_{n, i}^{\ell_{s-2}}\right)\right) \\
& \times\left(\sum_{j=2}^{\ell_{s}} \alpha_{n, j}^{\ell_{s-1}}\left(\rho^{j}\right)^{2} \prod_{i=1}^{j-1}\left(1-\alpha_{n, i}^{\ell_{s-1}}\right)\right) \alpha_{n, 1}^{s}\left\|t_{n}-q\right\|^{2}+\left(\rho^{j}\right)^{2}\left(\prod_{i=1}^{\ell_{2}}\left(1-\alpha_{n, i}^{1}\right)\left(\rho^{j}\right)^{2}\right) \\
& \times\left(\rho^{j}\right)^{2}\left(\prod_{i=1}^{\ell_{3}}\left(1-\alpha_{n, i}^{2}\right)\left(\rho^{j}\right)^{2}\right)\left(\rho^{j}\right)^{2}\left(\prod_{i=1}^{\ell_{4}}\left(1-\alpha_{n, i}^{3}\right)\left(\rho^{j}\right)^{2}\right) \times \cdots \times\left(\rho^{j}\right)^{2}\left(\prod_{i=1}^{\ell_{s-1}}\left(1-\alpha_{n, i}^{\ell_{s-2}}\right)\left(\rho^{j}\right)^{2}\right) \\
& \times\left(\rho^{j}\right)^{2}\left(\prod_{i=1}^{\ell_{s}}\left(1-\alpha_{n, i}^{\ell_{s-1}}\right)\left(\rho^{j}\right)^{2}\right)\left\|t_{n}-q\right\|^{2} \\
& <\alpha_{n, 1}^{1}\left\|t_{n}-q\right\|^{2}+\sum_{j=2}^{\ell_{2}} \alpha_{n, j}^{1} \prod_{i=1}^{j-1}\left(1-\alpha_{n, i}^{1}\right) \alpha_{n, 1}^{2}\left\|t_{n}-q\right\|^{2} \\
& +\prod_{i=1}^{\ell_{2}}\left(1-\alpha_{n, i}^{1}\right) \alpha_{n, 1}^{2}\left\|t_{n}-q\right\|^{2} \\
& +\left(\sum_{j=2}^{\ell_{2}} \alpha_{n, j}^{1} \prod_{i=1}^{j-1}\left(1-\alpha_{n, i}^{1}\right)\right)\left(1-\alpha_{n, 1}^{2}-\prod_{i=1}^{\ell_{3}}\left(1-\alpha_{n, i}^{2}\right)\right) \alpha_{n, 1}^{3}\left\|t_{n}-q\right\|^{2} \\
& +\left(\sum_{j=2}^{\ell_{2}} \alpha_{n, j}^{1} \prod_{i=1}^{j-1}\left(1-\alpha_{n, i}^{1}\right)\right)\left(\prod_{i=1}^{\ell_{3}}\left(1-\alpha_{n, i}^{2}\right)\right)\left\|t_{n}-q\right\|^{2} \\
& +\left(1-\alpha_{n, 1}^{2}-\prod_{i=1}^{\ell_{3}}\left(1-\alpha_{n, i}^{2}\right)\right)\left(\prod_{i=1}^{\ell_{2}}\left(1-\alpha_{n, i}^{1}\right)\right) \alpha_{n, 1}^{3}\left\|t_{n}-q\right\|^{2}
\end{aligned}
$$




$$
\begin{aligned}
& +\left(\prod_{i=1}^{\ell_{3}}\left(1-\alpha_{n, i}^{2}\right)\right)\left(\prod_{i=1}^{\ell_{2}}\left(1-\alpha_{n, i}^{1}\right)\right) \alpha_{n, 1}^{3}\left\|t_{n}-q\right\|^{2} \\
& +\left(\sum_{j=2}^{\ell_{2}} \alpha_{n, j}^{1} \prod_{i=1}^{j-1}\left(1-\alpha_{n, i}^{1}\right)\right)\left(1-\alpha_{n, 1}^{2}-\prod_{i=1}^{\ell_{3}}\left(1-\alpha_{n, i}^{2}\right)\right) \alpha_{n, 1}^{3}\left(1-\alpha_{n, 1}^{3}\right)\left\|t_{n}-q\right\|^{2} \\
& +\left(\sum_{j=2}^{\ell_{2}} \alpha_{n, j}^{1} \prod_{i=1}^{j-1}\left(1-\alpha_{n, i}^{1}\right)\right)\left(\prod_{i=1}^{\ell_{3}}\left(1-\alpha_{n, i}^{2}\right)\right) \alpha_{n, 1}^{3}\left(1-\alpha_{n, 1}^{3}\right)\left\|t_{n}-q\right\|^{2} \\
& \left.+\left(1-\alpha_{n, 1}^{2}-\prod_{i=1}^{\ell_{3}}\left(1-\alpha_{n, i}^{2}\right)\right)\right)\left(\prod_{i=1}^{\ell_{2}}\left(1-\alpha_{n, i}^{1}\right)\right) \alpha_{n, 1}^{3}\left(1-\alpha_{n, 1}^{3}\right)\left\|t_{n}-q\right\|^{2} \\
& +\left(\prod_{i=1}^{\ell_{3}}\left(1-\alpha_{n, i}^{2}\right)\right)\left(\prod_{i=1}^{\ell_{2}}\left(1-\alpha_{n, i}^{1}\right)\right) \alpha_{n, 1}^{3}\left(1-\alpha_{n, 1}^{3}\right)\left\|t_{n}-q\right\|^{2}+\cdots \\
& +\left(1-\alpha_{n, 1}^{1}-\prod_{i=1}^{\ell_{2}}\left(1-\alpha_{n, i}^{2}\right)\right)\left(1-\alpha_{n, 1}^{2}-\prod_{i=1}^{\ell_{3}}\left(1-\alpha_{n, i}^{2}\right)\right) \\
& \times\left(1-\alpha_{n, 1}^{3}-\prod_{i=1}^{\ell_{4}}\left(1-\alpha_{n, i}^{3}\right)\right) \times \cdots \times\left(1-\alpha_{n, 1}^{\ell_{s-2}}-\prod_{i=1}^{\ell_{s-1}}\left(1-\alpha_{n, i}^{\ell_{s-2}}\right)\right) \\
& \times\left(1-\alpha_{n, 1}^{\ell_{s-1}}-\prod_{i=1}^{\ell_{s}}\left(1-\alpha_{n, i}^{\ell_{s-1}}\right)\right) \alpha_{n, 1}^{s}\left\|t_{n}-q\right\|^{2}+\left(\prod_{i=1}^{\ell_{2}}\left(1-\alpha_{n, i}^{1}\right)\right) \\
& \times\left(\prod_{i=1}^{\ell_{3}}\left(1-\alpha_{n, i}^{2}\right)\right)\left(\prod_{i=1}^{\ell_{4}}\left(1-\alpha_{n, i}^{3}\right)\right) \times \cdots \times\left(\prod_{i=1}^{\ell_{s-1}}\left(1-\alpha_{n, i}^{\ell_{s-2}}\right)\right) \\
& \times\left(\prod_{i=1}^{\ell_{s}}\left(1-\alpha_{n, i}^{\ell_{s-1}}\right)\right)\left\|t_{n}-q\right\|^{2} \\
& <\alpha_{n, 1}^{1}\left\|t_{n}-q\right\|^{2}+\sum_{j=2}^{\ell_{2}} \alpha_{n, j}^{1} \prod_{i=1}^{j-1}\left(1-\alpha_{n, i}^{1}\right) \alpha_{n, 1}^{2}\left\|t_{n}-q\right\|^{2} \\
& +\prod_{i=1}^{\ell_{2}}\left(1-\alpha_{n, i}^{1}\right) \alpha_{n, 1}^{2}\left\|t_{n}-q\right\|^{2} \\
& +\left(\sum_{j=2}^{\ell_{2}} \alpha_{n, j}^{1} \prod_{i=1}^{j-1}\left(1-\alpha_{n, i}^{1}\right)\right) \alpha_{n, 1}^{3}\left(1-\alpha_{n, 1}^{2}\right)\left\|t_{n}-q\right\|^{2} \\
& +\alpha_{n, 1}^{3}\left(1-\alpha_{n, 1}^{2}\right)\left(\prod_{i=1}^{\ell_{2}}\left(1-\alpha_{n, i}^{1}\right)\right)\left\|t_{n}-q\right\|^{2} \\
& +\left(\sum_{j=2}^{\ell_{2}} \alpha_{n, j}^{1} \prod_{i=1}^{j-1}\left(1-\alpha_{n, i}^{1}\right)\right)\left(1-\alpha_{n, 1}^{2}\right) \alpha_{n, 1}^{3}\left(1-\alpha_{n, 1}^{3}\right)\left\|t_{n}-q\right\|^{2}
\end{aligned}
$$




$$
\begin{aligned}
& +\left(1-\alpha_{n, 1}^{2}\right)\left(\prod_{i=1}^{\ell_{2}}\left(1-\alpha_{n, i}^{1}\right)\right) \alpha_{n, 1}^{3}\left(1-\alpha_{n, 1}^{3}\right)\left\|t_{n}-q\right\|^{2}+\cdots+ \\
& +\left(1-\alpha_{n, 1}^{1}\right)\left(1-\alpha_{n, 1}^{2}\right)\left(1-\alpha_{n, 1}^{3}\right) \times \cdots \times\left(1-\alpha_{n, 1}^{\ell_{s-2}}\right) \\
& \times\left(1-\alpha_{n, 1}^{\ell_{s-1}}\right) \alpha_{n, 1}^{s}\left\|t_{n}-q\right\|^{2} \\
& =\alpha_{n, 1}^{1}\left\|t_{n}-q\right\|^{2}+\alpha_{n, 1}^{2}\left(1-\alpha_{n, 1}^{1}-\prod_{i=1}^{\ell_{2}}\left(1-\alpha_{n, i}^{1}\right)\right)\left\|t_{n}-q\right\|^{2} \\
& +\prod_{i=1}^{\ell_{2}}\left(1-\alpha_{n, i}^{1}\right) \alpha_{n, 1}^{2}\left\|t_{n}-q\right\|^{2} \\
& +\left(\left(1-\alpha_{n, 1}^{1}-\prod_{i=1}^{\ell_{2}}\left(1-\alpha_{n, i}^{1}\right)\right) \alpha_{n, 1}^{3}\left(1-\alpha_{n, 1}^{2}\right)\left\|t_{n}-q\right\|^{2}\right. \\
& +\alpha_{n, 1}^{3}\left(1-\alpha_{n, 1}^{2}\right)\left(\prod_{i=1}^{\ell_{2}}\left(1-\alpha_{n, i}^{1}\right)\right)\left\|t_{n}-q\right\|^{2} \\
& +\left(\left(1-\alpha_{n, 1}^{1}-\prod_{i=1}^{\ell_{2}}\left(1-\alpha_{n, i}^{1}\right)\right)\left(1-\alpha_{n, 1}^{2}\right) \alpha_{n, 1}^{3}\left(1-\alpha_{n, 1}^{3}\right)\left\|t_{n}-q\right\|^{2}\right. \\
& +\left(1-\alpha_{n, 1}^{2}\right)\left(\prod_{i=1}^{\ell_{2}}\left(1-\alpha_{n, i}^{1}\right)\right) \alpha_{n, 1}^{3}\left(1-\alpha_{n, 1}^{3}\right)\left\|t_{n}-q\right\|^{2}+\cdots+ \\
& +\left(1-\alpha_{n, 1}^{1}\right)\left(1-\alpha_{n, 1}^{2}\right)\left(1-\alpha_{n, 1}^{3}\right) \times \cdots \times\left(1-\alpha_{n, 1}^{\ell_{s-2}}\right) \\
& \times\left(1-\alpha_{n, 1}^{\ell_{s-1}}\right) \alpha_{n, 1}^{s}\left\|t_{n}-q\right\|^{2} \\
& <\left[\alpha_{n, 1}^{1}+\alpha_{n, 1}^{2}\left(1-\alpha_{n, 1}^{1}\right)+\left(1-\alpha_{n, 1}^{1}\right) \alpha_{n, 1}^{3}\left(1-\alpha_{n, 1}^{2}\right)+\left(1-\alpha_{n, 1}^{1}\right)\left(1-\alpha_{n, 1}^{2}\right) \alpha_{n, 1}^{3}\left(1-\alpha_{n, 1}^{3}\right)\right. \\
& +\cdots+\left(1-\alpha_{n, 1}^{1}\right)\left(1-\alpha_{n, 1}^{2}\right)\left(1-\alpha_{n, 1}^{3}\right) \times \cdots \times\left(1-\alpha_{n, 1}^{\ell_{s-2}}\right) \\
& \left.\times\left(1-\alpha_{n, 1}^{\ell_{s-1}}\right)\right]\left\|t_{n}-q\right\|^{2}
\end{aligned}
$$

(4.15) and (4.17) imply that

$$
\begin{aligned}
\left\|t_{n+1}-q\right\|^{2} \leq & \left\{\delta_{n, 1}+\left(1-\delta_{n, 1}\right)\left[\alpha_{n, 1}^{1}+\alpha_{n, 1}^{2}\left(1-\alpha_{n, 1}^{1}\right)+\left(1-\alpha_{n, 1}^{1}\right) \alpha_{n, 1}^{3}\left(1-\alpha_{n, 1}^{2}\right)\right.\right. \\
& +\left(1-\alpha_{n, 1}^{1}\right)\left(1-\alpha_{n, 1}^{2}\right)\left(1-\alpha_{n, 1}^{3}\right)+\cdots+\left(1-\alpha_{n, 1}^{1}\right)\left(1-\alpha_{n, 1}^{2}\right)\left(1-\alpha_{n, 1}^{3}\right) \\
& \left.\left.\times \cdots \times\left(1-\alpha_{n, 1}^{\ell_{s-2}}\right) \times\left(1-\alpha_{n, 1}^{\ell_{s-1}}\right)\right]\right\}\left\|t_{n}-q\right\|^{2}
\end{aligned}
$$

Using Lemma 2.3, we obtain (from (4.18)) that the sequence $\left\{x_{n}\right\}_{n=0}^{\infty}$ converges strongly to $q \in F(\Gamma)$.

Conversely, suppose $t_{n} \rightarrow q$ as $n \rightarrow \infty$. Then, we show that $\epsilon \rightarrow 0$ as $n \rightarrow \infty$. Indeed, from (3.5) with $v_{n}^{1}=y_{n}^{1},(4.12)$ and Proposition 2.4 with $u=q, t_{n}=t, j=i, k=1, \Gamma^{j-1} v_{n}^{1}=v_{j-1}$ and 
$\Gamma^{\ell_{1}} v_{n}^{1}=v_{n}$ we have

$$
\begin{aligned}
& \epsilon_{n}=\left\|t_{n+1}-\delta_{n, 1} t_{n}-\sum_{j=2}^{\ell_{1}} \delta_{n, j} \prod_{i=1}^{j-1}\left(1-\delta_{n, i}\right) \Gamma^{j-1} v_{n}^{1}-\prod_{i=1}^{\ell_{1}}\left(1-\delta_{n, i}\right) \Gamma^{\ell_{1}} v_{n}^{1}\right\|^{2} \\
&=\left\|t_{n+1}-q-\left(\delta_{n, 1} t_{n}+\sum_{j=2}^{\ell_{1}} \delta_{n, j} \prod_{i=1}^{j-1}\left(1-\delta_{n, i}\right) \Gamma^{j-1} v_{n}^{1}+\prod_{i=1}^{\ell_{1}}\left(1-\delta_{n, i}\right) \Gamma^{\ell_{1}} v_{n}^{1}-q\right)\right\|^{2} \\
& \leq\left\|t_{n+1}-q\right\|^{2}+\left\|\delta_{n, 1} t_{n}+\sum_{j=2}^{\ell_{1}} \delta_{n, j} \prod_{i=1}^{j-1}\left(1-\delta_{n, i}\right) \Gamma^{j-1} v_{n}^{1}+\prod_{i=1}^{\ell_{1}}\left(1-\delta_{n, i}\right) \Gamma^{\ell_{1}} v_{n}^{1}-q\right\|^{2} \\
& \leq\left\|t_{n+1}-q\right\|^{2}+\delta_{n, 1}\left\|t_{n}-q\right\|^{2}+\sum_{j=2}^{\ell_{1}} \delta_{n, j} \prod_{i=1}^{j-1}\left(1-\delta_{n, i}\right)\left\|\Gamma^{j-1} v_{n}^{1}-\Gamma^{j-1} q\right\|^{2} \\
&+\prod_{i=1}^{\ell_{1}}\left(1-\delta_{n, i}\right)\left\|\Gamma^{\ell_{1}} v_{n}^{1}-\Gamma^{\ell_{1}} q\right\|^{2} \\
& \leq\left\|t_{n+1}-q\right\|^{2}+\delta_{n, 1}\left\|t_{n}-q\right\|^{2}+\sum_{j=2}^{\ell_{1}} \delta_{n, j} \prod_{i=1}^{j-1}\left(1-\delta_{n, i}\right)\left(\rho^{j}\right)^{2}\left\|v_{n}^{1}-q\right\|^{2} \\
&+\prod_{i=1}^{\ell_{1}}\left(1-\delta_{n, i}\right)\left(\rho^{j}\right)^{2}\left\|v_{n}^{1}-q\right\|^{2} \\
&=\left\|t_{n+1}-q\right\|^{2}+\delta_{n, 1}\left\|t_{n}-q\right\|^{2}+\prod_{i=1}\left(1-\delta_{n, i}\right)\left(\rho^{j}\right)^{2}\left\|v_{n}^{1}-q\right\|^{2} \\
&\left.\left\|t_{n+1}-q\right\|^{2}+\delta_{n, 1}\left\|t_{n}-q\right\|^{2}+\left(1-\delta_{n, 1}\right)\left\|v_{n}^{1}-q\right\|^{2}\left(1-\delta_{n, i}\right)\right)\left(\rho^{j}\right)^{2}\left\|v_{n}^{1}-q\right\|^{2} \\
& i=1
\end{aligned}
$$

(4.17) and (4.19) imply

$$
\begin{aligned}
\epsilon_{n} \leq & \left\|t_{n+1}-q\right\|^{2}+\left\{\delta_{n, 1}+\left(1-\delta_{n, 1}\right)\left[\alpha_{n, 1}^{1}+\alpha_{n, 1}^{2}\left(1-\alpha_{n, 1}^{1}\right)\right.\right. \\
& +\left(1-\alpha_{n, 1}^{1}\right) \alpha_{n, 1}^{3}\left(1-\alpha_{n, 1}^{2}\right)+\left(1-\alpha_{n, 1}^{1}\right)\left(1-\alpha_{n, 1}^{2}\right) \alpha_{n, 1}^{3}\left(1-\alpha_{n, 1}^{3}\right) \\
& +\cdots+\left(1-\alpha_{n, 1}^{1}\right)\left(1-\alpha_{n, 1}^{2}\right)\left(1-\alpha_{n, 1}^{3}\right) \times \cdots \times\left(1-\alpha_{n, 1}^{\ell_{s-2}}\right) \\
& \left.\left.\times\left(1-\alpha_{n, 1}^{\ell_{s-1}}\right)\right]\right\}\left\|t_{n}-q\right\|^{2}
\end{aligned}
$$

Again, from our assumption, we obtain from (4.20) that $\epsilon_{n} \rightarrow 0$ as $n \rightarrow \infty$. Hence, the multistep $I H$-iteration scheme (3.1) is $\Gamma$-stable, and this completes the proof.

Remark 4.1. The following areas are still open: 
(i) to reconstruct, approximate the fixed points and the stability results of some existing iterative schemes in the current literature, other than the ones under study, for finite family of certain class of contractive-type map;

(ii) to compare convergent rates of the iterative schemes defined by (3.1) and (3.2) with those of (1.5) and (1.6).

\section{Competing interest}

The authors declare that there is no conflict of interest.

\section{REFERENCES}

[1] B. E. Rhoade, Fixed point theorems and stability results for fixed point iteration procedures, Indian J. Pure Appl. Math. 24(11) (1993) 691-03.

[2] B. E. Rhoade, Fixed point theorems and stability results for fixed point iteration procedures, Indian J. Pure Appl. Math. 21 (1990) 1-9.

[3] M. O. Osilike, A. Udoemene, A short proof of stability resultsfor fixed point iteration procedures for a class of contractive-type mappings, Indian J. Pure Appl. Math. 30 (1999) 122-1234.

[4] J. O. Olaleru, H. Akewe, An extension of Gregus fixed point theorem, Fixed Point Theory Appl. 2007 (2007) 78628. https://doi.org/10.1155/2007/78628.

[5] A. Ratiq, A convergence theprem for Mann's iteration procedure, Appl. Math. E-Note, 6 (2006) 289-293.

[6] H. Akewe, h. Olaoluwa, On the convergence of modified iteration process for generalised contractive-like operators, Bull. Math. Anal. Appl. 4(3) (2012) 78-86.

[7] B. E. Rhoade, A comparison of various definitions of contractive mappings, Trans. Amer. Math. Soc. 266 (1977) 257-290. https://doi.org/10.1090/s0002-9947-1977-0433430-4.

[8] B. E. Rhoade, Comments on two fixed point iteration methods, Trans. Amer. Math. Soc. 56 (1976) 741-750.

[9] B. E. Rhoade, Fixed point iteration using infinite matrices, Trans. Amer. Math. Soc. 196 (1974) 161-176. https: //doi .org/10.1090/S0002-9947-1974-0348565-1.

[10] V. Berinde, Iterative approximation of fixed points, Springer, Berlin, 2007.

[11] H. Akewe, Approximation of fixed and common fixed points of generalised contractive-like operators, PhD Thesis, University of Lagos, Nigeria, 2010.

[12] A. M. Harder, T. L. Hicks, Stability results for fixed point iterative procedures, Math. Jpn, 33(5) (1988) 693-706.

[13] A. M. Ostrowski, The round off stability of iterations, Z. Angew Math. Mech 47 (1967) 77-81.

[14] V. Berinde, On the stability of some fixed point problems, Bull. Stint. Univ. Bala Mare, Ser. B Fasc. Matinform. XVIII(1) 14 (2002) 7-14.

[15] T. Zamfirescu, Fixed point theorems in metric spaces, Arch. Math. 23 (1972) 292-298.

[16] M. O. Osilike, Stability results for Ishikawa fixed point iteration procedure, Indian J. Pure Appl. Math. 26(10) (1996) 937-941.

[17] M. O. Olutinwo, Some stability results for two hybrid fixed point iterative algorithms in normed linear space. Mat. Vesn, 61(4) (2009) 247-256.

[18] A. Ratiq, On the convergence of the three step iteration process in the class of quasi-contractive operators, Acta. Math. Acad. Paedagag Nayhazi, 22 (2006) 300-309.

[19] M. A. Noor, New approximation schemes for general variational inequalities, J. Math. Anal. Appl. 251 (2000) 217-229. https://doi.org/10.1006/jmaa.2000.7042. 
[20] W. A. Kirk, On successive approximations for nonexpansive mappings in Banach spaces, Glasg. Math. J. 12 (1971) 6-9.

[21] W. R. Mann, Mean value method in iteration, Proc. Amer. Math. Soc. 44 (2000) 506-510. https://doi.org/10. $2307 / 2032162$.

[22] S. Ishikawa, Fixed points by a new iteration methods, Proc. Amer. Math. Soc. 44 (1974) 147-150. https://doi. org/10.1090/S0002-9939-1974-0336469-5.

[23] C. O. Imoru, M. O. Olatinwo, On the stability of Picard's and Mann's iteration, Carpath. J. Math. 19 (2003) 155-160.

[24] R. Chugh, V. Kummar, Stability of hybrid fixed point iterative algorithm of Kirk-Noor-type in nonlinear spaces for self and nonself operators, Int. J. Contemp. Math. Sci. 7(24) (2012) 1165-1184.

[25] R. Chugh, V. Kummar, Strong convergence of SP iterative scheme for quasi-contractive operators, Int. J. Comput. Appl. 31(5) (2011) 21-27.

[26] H. Akewe, G. A. Okeeke, A. Olayiwola, Strong convergence and stability of Kirk-multistep-type iterative schemes for contractive-type operators, Fixed Point Theory Appl. 2014 (2014) 45. https://doi.org/10.1186/ $1687-1812-2014-45$.

[27] F. O. Isogugu, C. Izuchukwu, C. C. Okeke, New iteration scheme for approximating a common fixed point of a finite family of mappings, J. Math. 2020 (2020) 3287968. https://doi.org/10.1155/2020/3287968.

[28] I. K. Agwu, D. I. Igbokwe, New iteration algorithm for equilibrium problems and fixed point problems of two finite families of asymptotically demicontractive multivalued mappings, (in press). 